\title{
UNIVERSAL LOCAL PARAMETRIZATIONS VIA HEAT KERNELS AND EIGENFUNCTIONS OF THE LAPLACIAN
}

\author{
Peter W. Jones, Mauro Maggioni and Raanan Schul \\ Yale University, Department of Mathematics \\ 10 Hillhouse Ave, New Haven, CT 06510, U.S.A.; jones@math.yale.edu \\ Duke University, Department of Mathematics \\ Box 90320, Durham, NC 27708, U.S.A.; mauro.maggioni@duke.edu \\ Stony Brook University, Department of Mathematics \\ Stony Brook, NY 11794-3651, U.S.A.; schul@math.sunysb.edu
}

\begin{abstract}
We use heat kernels or eigenfunctions of the Laplacian to construct local coordinates on large classes of Euclidean domains and Riemannian manifolds (not necessarily smooth, e.g. with $\mathscr{C}^{\alpha}$ metric). These coordinates are bi-Lipschitz on embedded balls of the domain or manifold, with distortion constants that depend only on natural geometric properties of the domain or manifold. The proof of these results relies on estimates, from above and below, for the heat kernel and its gradient, as well as for the eigenfunctions of the Laplacian and their gradient. These estimates hold in the non-smooth category, and are stable with respect to perturbations within this category. Finally, these coordinate systems are intrinsic and efficiently computable, and are of value in applications.
\end{abstract}

\section{Introduction}

The concept of a coordinate chart for a manifold is quite old, but it has only recently become a subject of intensive study for data sets. In this paper we will state and prove a new theorem for coordinate charts on Riemannian manifolds. This result is meant to explain the empirically observed robustness of certain coordinate charts for data sets, Before stating our results, we explain in more detail the setting, first for manifolds, and then for data sets.

Let $\mathscr{M}$ be a Riemannian manifold. A coordinate chart (more precisely, a restriction of one) can be viewed as a mapping from a metric ball $B \subset \mathscr{M}$ into $\mathbf{R}^{d}$, where $d$ is the topological dimension of $\mathscr{M}$. This mapping has the form

$$
F(x)=\left(f_{1}(x), f_{2}(x), \ldots, f_{d}(x)\right) .
$$

It is natural to ask for $F$ to have low distortion. Let $F(B)=\tilde{B} \subset \mathbf{R}^{d}$. By assumption $F$ is a one to one mapping from $B$ to $\tilde{B}$. The Lipschitz norm of $F$ is defined as

$$
\|F\|_{\text {Lip }}=\sup _{\substack{x, y \in B \\ x \neq y}} \frac{\|F(x)-F(y)\|}{d_{\mathscr{M}}(x, y)}
$$

doi:10.5186/aasfm.2010.3508

2000 Mathematics Subject Classification: Primary 58J65, 35P99.

Key words: Heat kernel bounds, eigenfunction bounds, local charts, distortion estimates, biLipschitz mappings, non-linear dimension reduction. 
where $d_{\mathscr{M}}(\cdot, \cdot)$ is the metric on $\mathscr{M}$ and $\|\cdot\|$ is the usual Euclidean metric on $\mathbf{R}^{d}$. Similarly, one sets

$$
\left\|F^{-1}\right\|_{\text {Lip }}=\sup _{\substack{x, y \in B \\ x \neq y}} \frac{d_{\mathscr{M}}(x, y)}{\|F(x)-F(y)\|} .
$$

Then the distortion of $F$ on $B$ is defined to be

$$
\operatorname{Distortion}(F, B):=\|F\|_{\text {Lip }} \times\left\|F^{-1}\right\|_{\text {Lip }} \text {. }
$$

It is worth recalling at this point a prime example of a coordinate chart, namely the coordinate chart on a simply connected planar domain $\mathscr{D}$ given by a Riemann mapping $F$ from $\mathscr{D}$ to the unit disc $\mathbf{D}$. Let $z_{0} \in \mathscr{D}$ and define $r=\operatorname{dist}\left(z_{0}, \partial \mathscr{D}\right)$. If we choose our Riemann map $F$ to satisfy $F\left(z_{0}\right)=0$, then the distortion theorems of classical complex analysis (see e.g. [38, page 21]) state that $F$ maps the disc $B\left(z_{0}, \frac{r}{2}\right)$ onto "almost" the unit disc, with low distortion:

$$
\begin{aligned}
& B\left(0, \kappa^{-1}\right) \subset F\left(B\left(z_{0}, \frac{r}{2}\right)\right) \subset B\left(0,1-\kappa^{-1}\right), \\
& \text { Distortion }\left(F, B\left(z_{0}, \frac{r}{2}\right)\right) \leq \kappa
\end{aligned}
$$

In other words, on $B\left(z_{0}, \frac{r}{2}\right), F$ is a perturbation (in the proper sense) of the linear map given by $z \rightarrow F^{\prime}\left(z_{0}\right)\left(z-z_{0}\right)$, and $\left|F^{\prime}\left(z_{0}\right)\right| \sim \frac{1}{r}$.

In this paper we will look for an analogue of (1.0.2) and (1.0.3) above, but in the setting of Riemannian manifolds. We will show that on Riemannian manifolds of finite volume there is a locally defined $F$ that has these properties, and that this choice of $F$ will come from globally defined Laplacian eigenfunctions. On a metric embedded ball $B \subset \mathscr{M}$ we will choose global Laplacian eigenfunctions $\varphi_{i_{1}}, \varphi_{i_{2}}, \ldots, \varphi_{i_{d}}$ and constants $\gamma_{1}, \gamma_{2}, \ldots, \gamma_{d} \leq \kappa$ (for a universal constant $\kappa$ ) and define

$$
\Phi:=\left(\gamma_{1} \varphi_{i_{1}}, \gamma_{2} \varphi_{i_{2}}, \ldots, \gamma_{d} \varphi_{i_{d}}\right) .
$$

This choice of $\Phi$, depending heavily on $z_{0}$ and $r$, is globally defined, and on $B\left(z_{0}, \kappa^{-1} r\right)$ enjoys the same properties as the Riemann map does in (1.0.2) and (1.0.3). In other words, $\Phi$ maps $B\left(z_{0}, \kappa^{-1} r\right)$ to, roughly, a ball of unit size, with low distortion. Here we should point out the 1994 paper of Bérard et al. [5] where a weighted infinite sequence of eigenfunctions is shown to provide a global coordinate system (points in the manifold are mapped to $\ell_{2}$ ). To our knowledge this was the first result of this type in Riemannian geometry. Our results can be viewed as a strengthening of their work, and have as a consequence the statement that for a compact manifold without boundary, a good global coordinate system is given by the eigenfunctions $\varphi_{j}$ with eigenvalues $\lambda_{j}<\kappa R_{\text {inj }}^{-2}$. Here $R_{\text {inj }}$ is the inradius of $\mathscr{M}$, i.e. the largest $r>0$ such that for all $x \in \mathscr{M}, B(x, r)$ is an embedded ball.

The impetus for this paper and its results comes from certain recent results in the analysis of data sets. A recurrent idea is to approximate a data set, or a portion of it, lying in high dimensional space, by a manifold of low dimension, and find a parametrization of such data set or manifold. This process sometimes goes under the name of manifold learning, or linear or nonlinear dimensionality reduction. This type of work has been in part motivated by spectral graph theory [8] and spectral geometry $[7,23,17]$ (and references therein). Let $\left\{x_{j}\right\}_{1}^{N}$ be a collection of data points in a metric space $\mathbf{X}$. It is frequently quite difficult to extract any information from the data as it is presented. One solution is to embed the points in $\mathbf{R}^{n}$ for $n$ perhaps quite 
large, and then use linear methods (e.g. those using singular value decomposition) to obtain a dimensional reduction of the data set. In certain situations however linear methods are insufficient. For this reason, there has recently been great interest in nonlinear methods. ${ }^{1}$ Unfortunately such techniques seldomly come with guarantees on their capabilities of indeed finding local parametrization (but see, for example, $[18,19,52])$, or on quantitative statements on the quality of such parametrizations.

One of these methods, diffusion geometry, operates by first defining a kernel $K\left(x_{j}, x_{k}\right)$ on the data set, and then altering this slightly to obtain a self-adjoint matrix $\left(m_{j, k}\right)$ that roughly corresponds to the generator of a diffusion process. The eigenvectors of the matrix, should be seen as corresponding to Laplacian eigenfunctions on a manifold. One (judiciously) selects a collection $v_{i_{1}}, v_{i_{2}}, \ldots, v_{i_{m}}$ of eigenvectors and maps

$$
x_{k} \rightarrow\left(v_{i_{1}}, v_{i_{2}}, \ldots, v_{i_{m}}\right) \in \mathbf{R}^{m} .
$$

Careful choices of collections of eigenvectors have been empirically observed to give excellent representations of the data in a very low dimensional Euclidean space. What has been unclear is why this method should prove so successful. Our results show that in the case of Riemannian manifolds, one can prove that this philosophy is not just correct, but also robust. It is to be said that researchers so far have restricted their attention to the case when the lowest frequency eigenfunctions are selected, i.e. $i_{1}=1, i_{2}=2, \ldots, i_{m}=m[47,2,4,10,12,9]$.

Given these results, it is plausible to guess that an analogous result should hold for a local piece of a data set if that piece has in some sense a "local dimension" approximately $d$. There are certain difficulties with this philosophy. The first is that graph eigenfunctions are global objects and any definition of "local dimension" may change from point to point in the data set. A second difficulty is that our results for manifolds depend on classical estimates for eigenfunctions. This smoothness may be lacking in graph eigenfunctions.

It turns out that another of our manifold results does not suffer from these serious problems when working on a data set. We introduce simple "heat coordinate" systems on manifolds. Roughly speaking (and in the language of the previous paragraph) these are $d$ choices of manifold heat kernels that form a robust coordinate system on $B\left(z_{0}, \kappa^{-1} r\right)$. We call this method "heat triangulation" in analogy with triangulation as practiced in surveying, cartography, navigation, and modern GPS. Indeed our method is a simple translation of these classical triangulation methods, and has a closed formula on $\mathbf{R}^{d}$, which we note has infinite volume! (Our result on heat kernels makes no assumptions on the volume of the manifold.) For data sets, heat triangulation is a much more stable object than eigenfunction coordinates because:

- Heat kernels are local objects (see e.g. Proposition 3.3.2).

- If a manifold $\mathscr{M}$ is approximated by discrete sets $X$, the corresponding graph heat kernels converge rather nicely to the manifold heat kernel. This is studied for example in $[29,10,11,3]$.

\footnotetext{
${ }^{1}$ Examples of such disparate applications include document analysis [14], face recognition [25], clustering [35, 1], machine learning [4, 36, 50, 32, 31, 51], nonlinear image denoising and segmentation $[43,50]$, processing of articulated images [19], cataloguing of galaxies [20], pattern analysis of brain potentials [30] and EEG data [42], and the study of brain tumors [6]. A variety of algorithms for manifold learning have been proposed $[39,3,4,29,10,12,13,52,57,19,53,54,41,40]$.
} 
- One has good statistical control on smoothness of the heat kernel, simply because one can easily examine it and because one can use the Hilbert space $\left\{f \in L^{2}: \nabla f \in L^{2}\right\}$.

- Our results that use eigenfunctions rely in a crucial manner on Weyl's Lemma, whereas heat kernel estimates do not.

In a future paper we will return to applications of this method to data sets.

The philosophy used in this paper is as follows.

Step 1 . Find suitable points $y_{j}, 1 \leq j \leq d$ and a time $t$ so that the mapping given by heat kernels $\left(x \rightarrow K_{t}\left(x, y_{1}\right), \ldots, K_{t}\left(x, y_{d}\right)\right)$ is a good local coordinate system on $B\left(z, \kappa^{-1} r\right)$. (This is heat triangulation.)

Step 2. Use Weyl's Lemma to find suitable eigenfunctions $\varphi_{i_{j}}$ so that (with $K_{j}(x)=$ $\left.K_{t}\left(x, y_{j}\right)\right)$ one has large gradient.

Each point $y \in \mathscr{M}$ gives rise to a heat kernel $K_{t}(x, y)$. One may think of Step 1 as sampling this family of heat kernels $K_{t}(x, y)$ at $d$ different choices $y_{1}, \ldots, y_{d}$. Indeed, with high probability, randomly chosen points from the appropriate annulus will be suitable. Step 2 corresponds to sampling the vector $\left\{\varphi_{j}(x) e^{\lambda_{j} t}\right\}_{j} d$ times, once for each point $y_{1}, \ldots, y_{d}$. This last sampling, where we choose an index $j$, cannot be performed randomly! (See example in Section 5.1).

At this point we would like to note an advantage that local parametrization by eigenfunctions has over heat kernel triangulation (which we do not discuss in this paper). Consider the planar domain $[0,3 \epsilon] \times[0,3]$. Then, using only two Neumann eigenfunctions, one gets a good parametrization of the rectangle $[\epsilon, 2 \epsilon] \times[1,2]$. On the other hand, in order to get parametrization of similar distortion using heat kernel triangulation, on needs to use $\sim \frac{1}{\epsilon}$ different heat kernels.

To see where our philosophy comes from, we return for a moment to the setting of a simply connected planar domain $\mathscr{D}$ of area $=1$. Let $z_{0} \in \mathscr{D}$ and $r$ be as in the discussion before equation (1.0.2). With the choice of Riemann mapping $F$, with $F\left(z_{0}\right)=0$ we have the classical formula known to Riemann:

$$
F(z)=\exp \left\{-G\left(z, z_{0}\right)-i G^{*}\left(z, z_{0}\right)\right\} .
$$

Here $G\left(\cdot, z_{0}\right)$ is Green's function for the domain $\mathscr{D}$, with pole at $z_{0}$, and $G^{*}$ is the multivalued conjugate of $G$. Thus, all information about $F$ on $B\left(z_{0}, \frac{r}{2}\right)$ is encoded in $G\left(z, z_{0}\right)$. Recall that

$$
G\left(z, z_{0}\right)=\int_{0}^{\infty} K\left(z, z_{0}, t\right) d t
$$

where $K$ is the (Dirichlet) heat kernel for $\mathscr{D}$. Thus the behavior of $F$ can be read off the information on $K\left(z, z_{0}, t\right)$. Now write

$$
K\left(z, z_{0}, t\right)=\sum_{j=1}^{\infty} \varphi_{j}(z) \varphi_{j}\left(z_{0}\right) e^{\lambda_{j} t}
$$

where $\left\{\varphi_{j}\right\}$ is the collection of Dirichlet eigenfunctions (normalized to have $L^{2}$ norm $=1)$ and $\Delta \varphi_{j}=\lambda_{j} \varphi_{j}$. Notice that

$$
\left|F^{\prime}(z)\right|=\left|\nabla G\left(z, z_{0}\right)\right| e^{-G\left(z, z_{0}\right)} .
$$


Since $\left|F^{\prime}(z)\right| \sim \frac{1}{r}$ on $B\left(z_{0}, \frac{r}{2}\right)$, it is reasonable to guess from the above identities that there are eigenfunctions $\varphi_{j}$ such that

$$
\left|\nabla \varphi_{j}\right| \gtrsim \frac{1}{r}
$$

on $B\left(z_{0}, \kappa^{-1} r\right)$, for some $\kappa>1$, independent of $\mathscr{D}$. (More precisely, a short calculation with Weyl's estimates makes this reasonable.) This simple reasoning turns out to be correct and the main idea of this paper. The proof does not depend on any properties of holomorphic functions, but runs with equal ease in any dimension. This is because it only requires estimates on the heat kernel, Laplacian eigenfunctions and their derivatives, all of which are real variable objects.

The paper is organized in a top-bottom fashion, as follows. In Section 2 we state the main results, in Section 3 we present the main Lemmata, the proofs of the main results, and important estimates on the heat kernel and eigenfunctions of the Laplacian, together with their proofs, but mostly only in the Euclidean case. For the purpose of completeness we have recorded here proofs of several known estimates, over which the experts may wish to skip. In Section 3.5 we present the material for generalizing most estimates to the manifold case. Finally, we discuss some examples in Section 5.

\section{Results}

2.1. Euclidean domains. We first present the case of Euclidean domains. While our results in this setting follow from the more general results for manifolds discussed in the next section, the case of Euclidean domains is of independent interest, and the exposition of the main result as well as the proof in this case is simpler in the several technical respects.

We consider the heat equation in $\Omega$, a finite volume domain in $\mathbf{R}^{d}$, with either Dirichlet or Neumann boundary conditions i.e., respectively,

$$
\left\{\begin{array} { l } 
{ ( \Delta - \frac { \partial } { \partial t } ) u ( x , t ) = 0 , } \\
{ u | _ { \partial \Omega } = 0 , }
\end{array} \quad \text { or } \quad \left\{\begin{array}{l}
\left(\Delta-\frac{\partial}{\partial t}\right) u(x, t)=0 \\
\left.\partial_{\nu} u\right|_{\partial \Omega}=0
\end{array}\right.\right.
$$

Here $\nu$ is the outer normal on $\partial \Omega$. Independently of the boundary conditions, $\Delta$ denotes the Laplacian on $\Omega$. In this paper we restrict our attention to domains where the spectrum is discrete and the corresponding heat kernel can be written as

$$
K_{t}(z, w)=K_{t}^{\Omega}(z, w)=\sum_{j=0}^{+\infty} \varphi_{j}(z) \varphi_{j}(w) e^{-\lambda_{j} t} .
$$

where the $\left\{\varphi_{j}\right\}$ form an orthonormal basis of eigenfunctions of $\Delta$, with eigenvalues $0 \leq \lambda_{0} \leq \cdots \leq \lambda_{j} \leq \ldots$ We also require a (non-asymptotic) Weyl-type estimate: there is a constant $C_{\text {count }}$ such that for any $T>0$

$$
\#\left\{j: 0<\lambda_{j} \leq T\right\} \leq C_{\text {count }} T^{\frac{d}{2}}|\Omega| .
$$

In the Dirichlet case $C_{\text {count }}$ does not depend on $\Omega$ (see remark 3.4.3). For the Dirichlet case the only substantial problem is that the eigenfunctions may fail to vanish at the boundary. This in turn only occurs if there are boundary points where the Wiener series (for the boundary) converges [55, 28]. For the Neumann case the situation is more complicated $[34,26,33]$. In particular, there are domains with arbitrary closed 
continuous Neumann spectrum [26]. We therefore restrict ourselves in this paper to domains (and, later, manifolds) where conditions (2.1.1) and (2.1.2) are valid. More general boundary conditions can be handled in similar fashion, since our analysis is local and depends on the boundary conditions only through the properties above.

Finally, here and throughout the manuscript, we define $f_{B} f:=\frac{1}{|B|} \int_{B} f$.

Theorem 2.1.1. (Embedding via eigenfunctions, for Euclidean domains) Let $\Omega$ be a finite volume domain in $\mathbf{R}^{d}$, rescaled so that $|\Omega|=1$. Let $\Delta$ be the Laplacian in $\Omega$, with Dirichlet or Neumann boundary conditions, and assume that (2.1.1) and (2.1.2) hold. Then is a constant $\kappa>1$ that depends only on $d$ such that the following hold.

For any $z \in \Omega$, let $\rho \leq \operatorname{dist}(z, \partial \Omega)$. Then there exist integers $i_{1}, \ldots, i_{d}$ such that, if we let

$$
\gamma_{l}=\left(f_{B\left(z, \kappa^{-1} \rho\right)} \varphi_{i_{l}}^{2}\right)^{-\frac{1}{2}}, l=1, \ldots, d
$$

we have that

(a) the map

$$
\begin{aligned}
\Phi: B\left(z, \kappa^{-1} \rho\right) & \rightarrow \mathbf{R}^{d} \\
x & \mapsto\left(\gamma_{1} \varphi_{i_{1}}(x), \ldots, \gamma_{d} \varphi_{i_{d}}(x)\right)
\end{aligned}
$$

satisfies, for any $x_{1}, x_{2} \in B\left(z, \kappa^{-1} \rho\right)$,

$$
\frac{\kappa^{-1}}{\rho}\left\|x_{1}-x_{2}\right\| \leq \mid \Phi\left(x_{1}\right)-\Phi\left(x_{2}\right)\left\|\leq \frac{\kappa}{\rho}\right\| x_{1}-x_{2} \| ;
$$

(b) the associated eigenvalues satisfy

$$
\kappa^{-1} \rho^{-2} \leq \lambda_{i_{1}}, \ldots, \lambda_{i_{d}} \leq \kappa \rho^{-2}
$$

(c) the constants $\gamma_{l}$ satisfy

$$
\gamma_{1}, \ldots, \gamma_{d} \leq \kappa\left(C_{\text {count }}\right)^{\frac{1}{2}}
$$

Remark 2.1.2. In item (c) above, it will also be the case that $\kappa^{-1} \rho^{\frac{d}{2}} \leq \gamma_{j}$.

Remark 2.1.3. The dependence on $C_{\text {count }}$ is only needed in the Neumann case because, unlike the Dirichlet case, the upper bound in Weyl's Theorem depends on the domain. See Remark 3.4.3 for a more precise statement.

2.2. Manifolds with $\mathscr{C}^{\alpha}$ metric. The results above can be extended to certain classes of manifolds. In order to formulate a result corresponding to Theorem 2.1.1 we must first carefully define the manifold analogue of $\operatorname{dist}(z, \partial \Omega)$. Let $\mathscr{M}$ be a smooth, $d$-dimensional compact manifold, possibly with boundary. Suppose we are given a metric tensor $g$ on $\mathscr{M}$ which is $\mathscr{C}^{\alpha}$ for some $\alpha \in(0,1]$. For any $z_{0} \in \mathscr{M}$, let $(U, F)$ be a coordinate chart such that $z_{0} \in U$ and normalized so that

(i) $g^{i l}\left(F\left(z_{0}\right)\right)=\delta^{i l}$.

Then we assume that 
(ii) for any $x \in U$, and any $\xi, \nu \in \mathbf{R}^{d}$,

$$
\begin{aligned}
c_{\min }(g)\|\xi\|_{\mathbf{R}^{d}}^{2} & \leq \sum_{i, j=1}^{d} g^{i j}(F(x)) \xi_{i} \xi_{j} \text { and } \\
\sum_{i, j=1}^{d} g^{i j}(F(x)) \xi_{i} \nu_{j} & \leq c_{\max }(g)\|\xi\|_{\mathbf{R}^{d}}\|\nu\|_{\mathbf{R}^{d}} .
\end{aligned}
$$

We let

$$
r_{U}\left(z_{0}\right)=\sup \left\{r>0: B_{r}\left(F\left(z_{0}\right)\right) \subseteq F(U)\right\} .
$$

Observe that, when $g$ is at least $\mathscr{C}^{2}, r_{U}$ can be taken to be less than the inradius, with local coordinate chart $(U, F)$ given by the exponential map at $z$. The chart $(U, F)$ may intersect the boundary with no consequence, as all of the work will be done inside $B\left(z_{0}, r_{U}\right)$. We denote by $\|g\|_{\alpha}$ the maximum over all $i, j$ of

$$
\sup _{x \neq y} \frac{\left|g^{i j}(F(x))-g^{i j}(F(y))\right|}{|F(x)-F(y)|^{\alpha}}
$$

for $x, y$ in $U$. The natural volume measure $d \mu$ on the manifold is given, in any local chart, by $\sqrt{\operatorname{det} g}$; conditions (2.2.1) guarantee in particular that $\operatorname{det} g$ is uniformly bounded below from 0 . Let $\Delta_{\mathscr{M}}$ be the Laplace Beltrami operator on $\mathscr{M}$. In a local chart, we have

$$
\Delta_{\mathscr{M}} f(x)=-\frac{1}{\sqrt{\operatorname{det} g}} \sum_{i, j=1} \partial_{j}\left(\sqrt{\operatorname{det} g} g^{i j}(F(x)) \partial_{i} f\right)(F(x)),
$$

when $g$ is smooth enough (e.g. $g \in \mathscr{C}^{1}$ ). In general one defines the Laplacian through its associated quadratic form $[16,15]$. Conditions $(2.2 .1)$ are the usual uniform ellipticity conditions for the operator (2.2.3). With Dirichlet or Neumann boundary conditions, $\Delta_{\mathscr{M}}$ is self-adjoint on $L^{2}(\mathscr{M}, \mu)$. We will assume that the spectrum is discrete, denote by $0 \leq \lambda_{0} \leq \cdots \leq \lambda_{j} \leq$ its eigenvalues and by $\left\{\varphi_{j}\right\}$ the corresponding orthonormal basis of eigenfunctions, and write equations (2.1.1) and (2.1.2) with $\Omega$ replaced by $\mathscr{M}$.

Theorem 2.2.1. (Embedding via eigenfunctions, for manifolds) Let $(\mathscr{M}, g), z \in$ $\mathscr{M}$ be a $d$ dimensional manifold and $(U, F)$ be a chart as above. Assume $|\mathscr{M}|=1$. There is a constant $\kappa>1$, depending on $d, c_{\min }, c_{\max },\|g\|_{\alpha}, \alpha$ such that the following hold.

Let $\rho \leq r_{U}(z)$. Then there exist integers $i_{1}, \ldots, i_{d}$ such that if we let

$$
\gamma_{l}=\left(f_{B\left(z, \kappa^{-1} \rho\right)} \varphi_{i_{l}}^{2}\right)^{-\frac{1}{2}}, l=1, \ldots, d
$$

we have that

(a) the map

$$
\begin{aligned}
& \Phi: B\left(z, \kappa^{-1} \rho\right) \rightarrow \mathbf{R}^{d}, \\
& x \mapsto\left(\gamma_{1} \varphi_{i_{1}}(x), \ldots, \gamma_{d} \varphi_{i_{d}}(x)\right)
\end{aligned}
$$


satisfies for any $x_{1}, x_{2} \in B\left(z, \kappa^{-1} \rho\right)$

(b) the associated eigenvalues satisfy

$$
\frac{\kappa^{-1}}{\rho} d_{\mathscr{M}}\left(x_{1}, x_{2}\right) \leq\left\|\Phi\left(x_{1}\right)-\Phi\left(x_{2}\right)\right\| \leq \frac{\kappa}{\rho} d_{\mathscr{M}}\left(x_{1}, x_{2}\right) ;
$$

$$
\kappa^{-1} \rho^{-2} \leq \lambda_{i_{1}}, \ldots, \lambda_{i_{d}} \leq \kappa \rho^{-2}
$$

(c) the constants $\gamma_{l}$ satisfy

$$
\gamma_{1}, \ldots, \gamma_{d} \leq \kappa\left(C_{\text {count }}\right)^{\frac{1}{2}}
$$

Remark 2.2.2. As in the Euclidean case, in item (c) above, it will also be the case that $\kappa^{-1} \rho^{\frac{d}{2}} \leq \gamma_{j}$

Remark 2.2.3. Most of the proof is done on the local chart $(U, F)$ containing $z$. An inspection of the proof shows that we use only the norm $\|g\|_{\alpha}$ of the $g$ restricted to this chart.

Remark 2.2.4. When rescaling Theorem 2.2.1, it is important to note that if $f$ is a Hölder function with $\|f\|_{\mathscr{C}^{\alpha}}=A$ and $f_{r}(z)=f\left(r^{-1} z\right)$, then $\left\|f_{r}\right\|_{\mathscr{C}^{\alpha}}=A r^{\alpha}$. Since we will have $r<1, f_{r}$ satisfies a better Hölder estimate then $f$, i.e.

$$
\left\|f_{r}\right\|_{\mathscr{C}^{\alpha}}=A r^{\alpha}<A=\|f\|_{\mathscr{C}^{\alpha}} .
$$

We will repeatedly use this observation when discussing manifolds with $\mathscr{C}^{\alpha}$ metric.

Remark 2.2.5. We do not know, in both Theorem 2.1.1 and Theorem 2.2.1, whether it is possible to choose eigenfunctions such that $\gamma_{1} \sim \gamma_{2} \sim \ldots \sim \gamma_{d}$. If this were so, the map $x \mapsto\left(\varphi_{i_{1}}(x), \ldots, \varphi_{i_{d}}(x)\right)$ would be a low distortion map whose image has diameter $\geq \kappa^{-1}$.

Remark 2.2.6. As was noted by Guibas, when $\mathscr{M}$ has a boundary, in the case of Neumann boundary values, one may consider the "doubled" manifold, and may apply our result for a possibly larger $r_{U}(z)$.

Clearly Theorem 2.1.1 is a particular case of Theorem 2.2.1, but the proof of the former is significantly easier in that one can use standard estimates on eigenfunctions of the Laplacian and their derivatives. For the sake of presentation we present one proof for both Theorems, but two sets of required Lemmata for those estimates which are significantly different in the two cases.

Remark 2.2.7. The method of the proofs also gives a result independent of the constant $C_{\text {count }}$ : Let $(\mathscr{M}, g)$ and $z \in \mathscr{M}$ be as in Theorem 2.2.1. Let $\eta>0$, and assume that for any $x \in \mathscr{M}$ we have a chart $(U, F)$ such that $r_{U}(x) \geq \eta>0$ (in particular, $\mathscr{M}$ has no boundary). Then for $\rho \leq \eta$ the same results as in Theorem 2.2.1 hold, except the constant $\kappa$ depends only on $d, c_{\min }, c_{\max },\|g\|_{\alpha}, \alpha$ and not on $C_{\text {count }}$. This is due to the fact that $C_{\text {count }}$ becomes universal for values of $T>\eta^{-2}$.

Another, in some sense stronger, result is true. One may replace the $d$ eigenfunctions in Theorem 2.2.1 by $d$ heat kernels $\left\{K_{t}\left(z, y_{i}\right)\right\}_{i=1, \ldots, d}$. In fact such heat kernels arise naturally in the main steps of the proofs of Theorem 2.1.1 and Theorem 2.2.1. This leads to an embedding map with even stronger guarantees:

Theorem 2.2.8. (Heat Triangulation Theorem) Let $(\mathscr{M}, g), z \in \mathscr{M}$ and $(U, F)$ be as above, with the exception we now make no assumptions on the finiteness of 
the volume of $\mathscr{M}$ and the existence of $C_{\text {count }}$. Let $\rho \leq r_{U}(z)$. Let $p_{1}, \ldots, p_{d}$ be $d$ linearly independent directions. There are constants $c>0$ and $c^{\prime}, \kappa>1$, depending on $d, c_{\min }, c_{\max }, \rho^{\alpha}\|g\|_{\alpha}, \alpha$, and the smallest and largest eigenvalues of the Gramian matrix $\left(\left\langle p_{i}, p_{j}\right\rangle\right)_{i, j=1, \ldots, d}$, such that the following holds. Let $y_{i}$ be so that $y_{i}-z$ is in the direction $p_{i}$, with $c \rho \leq d_{\mathscr{M}}\left(y_{i}, z\right) \leq 2 c \rho$ for each $i=1, \ldots, d$ and let $t=\kappa^{-1} \rho^{2}$. The map

$$
\left.x \mapsto\left(\rho^{d} K_{t}\left(x, y_{1}\right)\right), \ldots, \rho^{d} K_{t}\left(x, y_{d}\right)\right)
$$

satisfies, for any $x_{1}, x_{2} \in B\left(z, \kappa^{-1} \rho\right)$,

$$
\frac{\kappa^{-1}}{c^{\prime} \rho} d_{\mathscr{M}}\left(x_{1}, x_{2}\right) \leq\left\|\Phi\left(x_{1}\right)-\Phi\left(x_{2}\right)\right\| \leq \frac{\kappa c^{\prime}}{\rho} d_{\mathscr{M}}\left(x_{1}, x_{2}\right) .
$$

The reason for the factor $\rho^{\alpha}$ which we have in $\rho^{\alpha}\|g\|_{\alpha}$ above is to get scaling invariance.

This theorem holds for the manifold and Euclidean case alike, and depends only on the heat kernel estimates (and its gradient). We again note that for this particular Theorem we require no statement about the volume of the manifold, the existence of $L^{2}$ Laplacian eigenfunctions, or their number. The constants for the Euclidean case, depend only on dimension, and not on the domain. The content of this theorem is that one is able to choose the directions $y_{i}-z$ randomly on a sphere, and with high probability on gets a low distortion map. This gives rise to a sampling theorem.

One may replace the (global) heat kernel above with a local heat kernel, i.e. the heat kernel for the ball $B(z, \rho)$ with the metric induced by the manifold and Dirichlet boundary conditions. In fact, this is a key idea in the proof of all of the above Theorems. Thus, on the one hand our results are local, i.e. independent of the global geometry of the manifold, yet on the other hand they are in terms of global eigenfunctions.

As is clear from the proof, all theorems hold for more general boundary conditions. This is especially true for the Heat Triangulation Theorem, which does not even depend on the existence of a spectral expansion for the heat kernel.

Example 2.2.9. It is a simple matter to verify this Theorem for the case where the manifold in $\mathbf{R}^{d}$. For example if $d=2, \rho=1$, and $z=0, y_{1}=(-1,0)$ and $y_{2}=(0,-1)$. Then if $K_{t}(x, y)$ is the Euclidean heat kernel,

$$
x \rightarrow\left(K_{1}\left(x, y_{1}\right), K_{1}\left(x, y_{2}\right)\right)
$$

is a (nice) biLipschitz map on $B\left((0,0), \frac{1}{2}\right)$. (The result for arbitrary radii then follows from a scaling argument). This is because on can simply evaluate the heat kernel

$$
K_{t}(x, y)=\frac{1}{4 \pi t} e^{-\frac{|x-y|^{2}}{4 t}} .
$$

So in $B_{\frac{1}{2}}((0,0))$

$$
\nabla K_{1}\left(x, y_{1}\right) \sim \frac{1}{2 \pi} e^{-\frac{1}{4}}(1,0) \text { and } \nabla K_{1}\left(x, y_{2}\right) \sim \frac{1}{2 \pi} e^{-\frac{1}{4}}(0,1) .
$$

Acknowledgments. The authors would like to thank K. Burdzy, R. R. Coifman, P. Gressman, N. Tecu, H. Smith and A. D. Szlam for useful discussions during the preparation of the manuscript, as well as IPAM for hosting these discussions and more. P. W. Jones is grateful for partial support from NSF DMS 0501300. M. Maggioni is grateful for partial support from NSF DMS 0650413, NSF CCF 0808847 and 
ONR N00014-07-1-0625. R. Schul is grateful for partial support from NSF DMS 0502747. The main theorems were reported in the announcement [27].

\section{The proof of Theorems 2.1.1 and 2.2.1}

The proofs in the Euclidean and manifold case are similar. In this section we present the steps of the proofs of Theorems 2.1.1, 2.2.1 we will postpone the technical estimates needed to later sections.

Because we may change base points, we will use $R_{z}$ (or similarly, $R_{w}$ ) in place of $\rho$. We will also interchange between $B(x, r)$ and $B_{r}(x)$.

Remark 3.0.10. (Some remarks about the manifold case)

(a) As mentioned in Remark 2.2.3, we will often restrict to working on a single (fixed!) chart in local coordinates. When we discuss moving in a direction $p$, we mean in the local coordinates.

(b) We will use Brownian motion arguments (on the manifold). In order to have existence and uniqueness one needs smoothness assumptions on the metric (say, $\mathscr{C}^{2}$, albeit less would suffice, see e.g. [37]). Therefore we will first prove the Theorem in the manifold case in the $\mathscr{C}^{2}$ metric category, and then use perturbation estimates to obtain the result for $g \in \mathscr{C}^{\alpha}$. To this end, we will often have dependence on $\|g\|_{\alpha}$ even though we will be (for a specific Lemma or Proposition) assuming the $g \in \mathscr{C}^{2}$.

Notation. In what follows, we will write $f(x) \lesssim_{c_{1}, \ldots, c_{n}} g(x)$ if there exists a constant $C$ depending only on $c_{1}, \ldots, c_{n}$, and not on $f, g$ or $x$, such that $f(x) \leq$ $C g(x)$ for all $x$ (in a specified domain). We will write $f(x) \sim_{c_{1}, \ldots, c_{n}} g(x)$ if both $f(x) \lesssim_{c_{1}, \ldots, c_{n}} g(x)$ and $g(x) \lesssim_{c_{1}, \ldots, c_{n}} f(x)$. If $f, g$ take values in $\mathbf{R}^{d}$ the inequalities are intended componentwise. We will write $a \sim_{C_{1}}^{C_{2}} b$ if $C_{1} b \leq a \leq C_{2} b$ (componentwise for $a, b$ vectors).

In what follows we will write $\partial_{p} K_{t}(\cdot, \cdot)$ to denote the partial derivative with respect to the second variable of a heat kernel at time $t$.

3.1. The Case of $g \in \mathscr{C}^{2}$. We note that even though we assume $g \in \mathscr{C}^{2}$, we only use the $\mathscr{C}^{\alpha}$ norm of $g$. The idea of the proof of Theorems 2.1.1 and 2.2.1 is as follows. We start by fixing a direction $p_{1}$ at $z$. We would like to find an eigenfunction $\varphi_{i_{1}}$ such that $\left|\partial_{p_{1}} \varphi_{i_{1}}\right| \gtrsim R_{z}^{-1}$ on $B_{c_{1} R_{z}}(z)$. In order to achieve this, we start by showing that the heat kernel has large gradient in an annulus of inner and outer radius $\sim R_{z}^{-1}$ around $y_{1}$ ( $y_{1}$ chosen such that $z$ is in this annulus, in direction $\left.p_{1}\right)$. We then show that the heat kernel and its gradient can be approximated on this annulus by the partial sum of (2.1.1) over eigenfunctions $\varphi_{\lambda}$ which satisfy both $\lambda \sim R_{z}^{-2}$ and $R_{z}^{-\frac{d}{2}}\left\|\varphi_{\lambda}\right\|_{L^{2}\left(B_{c_{1} R_{z}}(z)\right)} \gtrsim 1$. By the pigeon-hole principle, at least one such eigenfunction, let it be $\varphi_{i_{1}}$ has a large partial derivative in the direction $p_{1}$. We then consider $\nabla \varphi_{i_{1}}$ and pick $p_{2} \perp \nabla \varphi_{i_{1}}$ and by induction we select $\varphi_{i_{1}}, \ldots, \varphi_{i_{d}}$, making sure that at each stage we can find $\varphi_{i_{k}}$, not previously chosen, satisfying $\left|\partial_{p_{k}} \varphi_{i_{k}}\right| \sim R_{z}^{-1}$ on $B_{c_{1} R_{z}}(z)$. We finally show that for the proper choice of constants $\gamma_{1}, . ., \gamma_{d} \lesssim 1$, the map $\Phi:=\left(\gamma_{1} \varphi_{i_{1}}, \ldots, \gamma_{d} \varphi_{i_{d}}\right)$ satisfies the desired properties.

When working on a manifold, we assume in what follows that we fix a local chart containing $B_{R_{z}}(z)$, as at the beginning of section 2.2.

Step 1. Estimates on the heat kernel and its gradient. Let $K$ be the Dirichlet or Neumann heat kernel on $\Omega$ or $\mathscr{M}$, corresponding to one of the Laplacian operators considered above associated with $g$ and the fixed $\alpha$. 
Assumption A.1. Assume $g \in \mathscr{C}^{2}$, and let $\alpha \in(0,1]$ be given and fixed. Let constants $\delta_{0}, \delta_{1}>0$ depend on $d, c_{\min }, c_{\max },\|g\|_{\alpha}, \alpha$. We consider $z, w \in \Omega$ satisfying $\frac{\delta_{1}}{2} R_{z}<t^{\frac{1}{2}}<\delta_{1} R_{z}$ and $|z-w|<\delta_{0} R_{z}$.

Remark 3.1.1. Proposition 3.1.2 below makes no assumptions on the finiteness of the volume of $\mathscr{M}$ and the existence of $C_{\text {count }}$. It is also used in the proof of Theorem 2.2.8.

Proposition 3.1.2. Assume Assumption A.1, $\delta_{0}$ sufficiently small, and $\delta_{1}$ is sufficiently small depending on $\delta_{0}$. Then there are constants $C_{1}, C_{2}, C_{1}^{\prime}, C_{2}^{\prime}, C_{9}>0$, that depend on $d, \delta_{0}, \delta_{1}, c_{\min }, c_{\max }, R_{z}^{\alpha}\|g\|_{\alpha}, \alpha$, such that the following hold:

(i) the heat kernel satisfies

$$
K_{t}(z, w) \sim_{C_{1}}^{C_{2}} t^{\frac{-d}{2}}
$$

(ii) if $\frac{1}{2} \delta_{0} R_{z}<|z-w|, p$ is a unit vector in the direction of $z-w$, and $q$ is arbitrary unit vector, then

$$
\begin{gathered}
\left|\nabla K_{t}(z, w)\right| \sim_{C_{1}^{\prime}}^{C^{\prime}} t^{\frac{-d}{2}} \frac{R_{z}}{t} \text { and }\left|\partial_{p} K_{t}(z, w)\right| \sim_{C_{1}^{\prime}}^{C^{\prime}} t^{\frac{-d}{2}} \frac{R_{z}}{t} \\
\left|\partial_{q} K_{t}(z, w)-\partial_{q} K_{t}^{\mathbf{R}^{d}}(z, w)\right| \leq C_{9} t^{\frac{-d}{2}} \frac{R_{z}}{t}
\end{gathered}
$$

where $C_{9} \rightarrow 0$ as $\delta_{1} \rightarrow 0$ (with $\delta_{0}$ fixed); here, $K_{t}^{\mathbf{R}^{d}}(z, w)$ is the usual Euclidean heat kernel;

(iii) if $\frac{1}{2} \delta_{0} R_{z}<|z-w|$, and $q$ is as above, then for $s \leq t$,

$$
K_{s}(z, w) \lesssim_{C_{2}} t^{\frac{-d}{2}},\left|\nabla K_{s}(z, w)\right| \lesssim_{C_{2}^{\prime}} t^{\frac{-d}{2}} \frac{R_{z}}{t} \text { and }\left|\partial_{q} K_{s}(z, w)\right| \lesssim_{C_{2}^{\prime}} t^{\frac{-d}{2}} \frac{R_{z}}{t}
$$

(iv) $C_{1}, C_{2}$ both tend to a single function of $d, c_{\min }, c_{\max },\|g\|_{\alpha}, \alpha$, as $\delta_{1}$ tends to 0 with $\delta_{0}$ fixed;

The reason for the factor of $R_{z}^{\alpha}$ which we have in $R_{z}^{\alpha}\|g\|_{\alpha}$ above is to get scaling invariance. Proposition 3.1.2 is proved in subsection 3.3.1 for the Euclidean case and in subsection 3.5.3.

We continue with the proof of Theorem 2.1.1 and 2.2.1. From here on, unless explicitly stated, we assume the existence $C_{\text {count }}$. We have the spectral expansion

$$
K_{t}(x, y)=\sum_{j=0}^{+\infty} e^{-\lambda_{j} t} \varphi_{j}(x) \varphi_{j}(y) .
$$

Remark 3.1.3. The assumptions of Theorems 2.1 .1 and 2.2.1 say that $|\mathscr{M}|=1$ (manifold case) or $|\Omega|=1$ (Euclidean domain case). Thus, unless explicitly stated, we will assume in the lemmata below that we have $R_{z} \lesssim_{d, c_{\min }, c_{\max },\|g\|_{\alpha}, \alpha} 1$.

The following steps aim at replacing appropriately chosen heat kernels by a set of eigenfunctions, by extracting the "leading terms" in their spectral expansion.

Step 2. Heat kernel and eigenfunctions. We start by restricting our attention to eigenfunctions which do not have too high frequency. Let

$$
\Lambda_{L}(A)=\left\{\lambda_{j}: \lambda_{j} \leq A t^{-1}\right\} \quad \text { and } \quad \Lambda_{H}\left(A^{\prime}\right)=\left\{\lambda_{j}: \lambda_{j}>A^{\prime} t^{-1}\right\}=\Lambda_{L}\left(A^{\prime}\right)^{c} .
$$


A first connection between the heat kernel and eigenfunctions is given by the following truncation Lemma, which is proved in Subsection 3.4.2.

Lemma 3.1.4. Under Assumption A.1, for $A>1$ large enough and $A^{\prime}<1$ small enough, depending on $\delta_{0}, \delta_{1}, C_{1}, C_{2}, C_{1}^{\prime}, C_{2}^{\prime}$ (as in Proposition 3.1.2), there exist constants $C_{3}, C_{4}$ (depending on $A, A^{\prime}$ as well as $d, c_{\min }, c_{\max },\|g\|_{\alpha}, \alpha$ ) such that

(i) the heat kernel is approximated by the truncated expansion

$$
K_{t}(z, w) \sim_{C_{3}}^{C_{4}} \sum_{j \in \Lambda_{L}(A)} \varphi_{j}(z) \varphi_{j}(w) e^{-\lambda_{j} t}
$$

(ii) if $\frac{1}{2} \delta_{0} R_{z}<|z-w|$, and $p$ is a unit vector parallel to $z-w$, then

$$
\partial_{p} K_{t}(z, w) \quad \sim_{C_{3}}^{C_{4}} \sum_{j \in \Lambda_{L}(A) \cap \Lambda_{H}\left(A^{\prime}\right)} \varphi_{j}(z) \partial_{p} \varphi_{j}(w) e^{-\lambda_{j} t}
$$

Furthermore,

$$
\left\|\sum_{j \notin \Lambda_{L}(A) \cap \Lambda_{H}\left(A^{\prime}\right)} \varphi_{j}(z) \nabla \varphi_{j}(w) e^{-\lambda_{j} t}\right\| \leq C_{10} t^{\frac{-d}{2}} \frac{R_{z}}{t},
$$

where $C_{10} \rightarrow 0$ as $A \rightarrow \infty$ and $A^{\prime} \rightarrow 0$;

(iii) $C_{3}, C_{4}$ both tend to 1 as $A \rightarrow \infty$ and $A^{\prime} \rightarrow 0$.

This Lemma implies that in the heat kernel expansion we do not need to consider eigenfunctions corresponding to eigenvalues larger than $A t^{-1}$. However, in our search for eigenfunctions with the desired properties, we need to restrict our attention further, by discarding eigenfunctions that have too small a gradient around $z$. Let

$$
\begin{aligned}
& \Lambda_{E}\left(p, z, R_{z}, \delta_{0}, c_{0}\right) \\
& :=\left\{\lambda_{j} \in \sigma(\Delta): \frac{1}{c_{0}} R_{z}\left|\partial_{p} \varphi_{j}(z)\right| \geq\left(f_{B\left(z, \frac{1}{2} \delta_{0} R_{z}\right)} \varphi_{j}\left(z^{\prime}\right)^{2} d z^{\prime}\right)^{\frac{1}{2}}\right\} .
\end{aligned}
$$

Here and in what follows, $f_{A} f=|A|^{-1} \int_{A} f$. The truncation Lemma 3.1.4 can be strengthened, on average, into

Lemma 3.1.5. Assume Assumption A.1, $\delta_{0}$ sufficiently small, and $\delta_{1}$ is sufficiently small depending on $\delta_{0}$. For $C_{3}, C_{4}$ close enough to 1 (as in Lemma 3.1.4), and $c_{0}$ small enough (depending on $C_{2}, C_{1}^{\prime}, \delta_{0}, \delta_{1}$ ) there exist constants $C_{5}, C_{6}$ (depending only on $C_{3}, C_{4}, C_{9}$, and $\left.c_{0}\right)$ such that if $\frac{1}{2} \delta_{0} R_{z}<|z-w|$, and $p$ is a unit vector parallel to $z-w$, then

$$
\left|\partial_{p} K_{t}(w, z)\right| \sim_{C_{5}}^{C_{6}}\left|\sum_{\lambda_{j} \in \Lambda_{L}(A) \cap \Lambda_{H}\left(A^{\prime}\right) \cap \Lambda_{E}\left(z, R_{z}, \delta_{0}, c_{0}\right)} \varphi_{j}(w) \partial_{p} \varphi_{j}(z) e^{-\lambda_{j} t}\right| .
$$

Step 3. Choosing appropriate eigenfunctions. Define the constants $\gamma_{\varphi_{j}}$ as

$$
\gamma_{\varphi_{j}}=\left(f_{B_{\frac{1}{2} \delta_{0} R_{z}}(z)} \varphi_{j}\left(z^{\prime}\right)^{2} d z^{\prime}\right)^{-\frac{1}{2}}
$$


Note that since $\varphi_{j}$ is $L^{2}$ normalized, we have $\gamma_{\varphi_{j}} \gtrsim R_{z}^{d / 2}$. A subset of these constants and corresponding eigenfunctions will soon be chosen to give us the constants $\left\{\gamma_{j}\right\}$ and corresponding eigenfunctions $\left\{\varphi_{i_{j}}\right\}$ in the statement of Theorem 2.1.1 and Theorem 2.2.1.

The set of eigenfunctions with eigenvalues in $\Lambda_{L}(A) \cap \Lambda_{H}\left(A^{\prime}\right) \cap \Lambda_{E}\left(p, z, R_{z}, \delta_{0}, c_{0}\right)$ is well-suited for our purposes, in view of the following:

Lemma 3.1.6. Under Assumption A.1, for $\delta_{0}$ small enough, there exists a constant $C_{7}$ depending on $c_{0}$ and $C_{8}$ depending on $\left\{\delta_{0}, c_{\min }, c_{\max },\|g\|_{\alpha}, \alpha\right\}$ and a constant $b>0$ which depends on $c_{0}, d, c_{\min }, c_{\max },\|g\|_{\alpha}, \alpha$ such that the following holds. Let $p$ be a direction. For all $j \in \Lambda_{E}\left(p, z, R_{z}, \delta_{0}, c_{0}\right)$ we have that for all $z^{\prime}$ such that $\left\|z-z^{\prime}\right\| \leq b \delta_{0} R_{z}$

$$
\left|\partial_{p} \varphi_{j}\left(z^{\prime}\right)\right| \sim_{C_{7}}^{C_{8}} R_{z}^{-1}\left(f_{B_{\frac{1}{2} \delta_{0} R_{z}}(z)} \varphi_{j}^{2}\right)^{\frac{1}{2}} .
$$

Moreover, there exists an index $j \in \Lambda_{L}(A) \cap \Lambda_{H}\left(A^{\prime}\right) \cap \Lambda_{E}\left(p, z, R_{z}, \delta_{0}, c_{0}\right)$, so that we have

$$
\gamma_{\varphi_{j}} \lesssim\left(C_{\text {count }}\right)^{\frac{1}{2}}
$$

with constants depending on $A, C_{1}, C_{1}^{\prime}, C_{2}, C_{2}^{\prime}, C_{6},\left\{d, c_{\min }, c_{\max },\|g\|_{\alpha}, \alpha\right\}, \delta_{0}, \delta_{1}$.

We can now complete the proof of Theorems 2.1.1, 2.2.1.

Proof of Theorems 2.1.1 and 2.2.1 for the case $g \in \mathscr{C}^{2}$. Lemma 3.1.6 yields an eigenfunction that serves our purpose in a given direction. To complete the proof of the Theorems, we need to cover $d$ linearly independent directions. Pick an arbitrary direction $p_{1}$. By Lemma 3.1.6 we can find $j_{1} \in \Lambda_{L}(A) \cap \Lambda_{H}\left(A^{\prime}\right) \cap \Lambda_{E}\left(p, z, R_{z}, \delta_{0}, c_{0}\right)$, (in particular $j_{1} \sim t^{-1}$ ) such that $\left|\gamma_{\varphi_{j_{1}}} \partial_{p_{1}} \varphi_{j_{1}}(z)\right| \geq c_{0} R_{z}^{-1}$. Let $p_{2}$ be a direction orthogonal to $\nabla \varphi_{j_{1}}(z)$. We apply again Lemma 3.1.6, and find $j_{2}<A t^{-1}$ so that $\left|\gamma_{\varphi_{j_{2}}} \partial_{p_{2}} \varphi_{j_{2}}(z)\right| \geq c_{0} R_{z}^{-1}$. Note that necessarily $j_{2} \neq j_{1}$ and $p_{2}$ is linearly independent of $p_{1}$. In fact, by choice of $p_{2}$,

$$
\partial_{p_{2}} \varphi_{j_{1}}=0
$$

We proceed in this fashion. By induction, once we have chosen $j_{1}, \ldots, j_{k}(k<d)$, and the corresponding $p_{1}, \ldots, p_{k}$, such that $\left|\gamma_{\varphi_{j_{l}}} \partial_{p_{l}} \varphi_{j_{l}}(z)\right| \geq c_{0} R_{z}^{-1}$, for $l=1, \ldots, k$, we pick $p_{k+1}$ orthogonal to $\left\langle\left\{\nabla \varphi_{j_{n}}(z)\right\}_{n=1, \ldots, k}\right\rangle$ and apply Lemma 3.1.6, that yields $j_{k+1}$ such that $\left|\gamma_{\varphi_{j_{k+1}}} \partial_{p_{k+1}} \varphi_{j_{k+1}}(z)\right| \geq c_{0} R_{z}^{-1}$.

From here on we denote by $\gamma_{i}=\gamma_{\varphi_{j_{i}}}$ for simplicity of notation. These are the constants $\left\{\gamma_{i}\right\}$ appearing in the statement of Theorem 2.1.1 and Theorem 2.2.1.

We claim that the matrix

$$
A_{k+1}:=\left(\gamma_{m} \partial_{p_{n}} \varphi_{j_{m}}(z)\right)_{m, n=1, \ldots, k+1}
$$

is lower triangular and $\left\{p_{1}, \ldots, p_{k+1}\right\}$ is linearly independent. Lower-triangularity of the matrix follows by induction and the choice of $p_{k+1}$. Assume $\sum_{n=1}^{k+1} a_{n} p_{n}=0$, then $\left\langle\sum_{n=1}^{k+1} a_{n} p_{n}, \gamma_{l} \nabla \varphi_{j_{l}}(z)\right\rangle=0$ for all $l=1, \ldots, k+1$, i.e. $a \in \mathbf{R}^{k+1}$ solves the linear system

$$
A_{k+1} a=0 .
$$

But $A_{k+1}$ is lower triangular with all diagonal entries non-zero, hence $a=0$. 
For $l \leq k$ we have $\left\langle\nabla \varphi_{j_{l}}(z), p_{k+1}\right\rangle=0$ and, by Lemma 3.1.6,

$$
\left|\left\langle\gamma_{l} \nabla \varphi_{j_{l}}, p_{l}\right\rangle\right| \gtrsim R_{z}^{-1} .
$$

Now let $\Phi_{k}=\left(\gamma_{1} \varphi_{j_{1}}, \ldots, \gamma_{k} \varphi_{j_{k}}\right)$ and $\Phi=\Phi_{d}$. We start by showing that

$$
\left\|\left.\nabla \Phi\right|_{z}(w-z)\right\| \gtrsim_{d} \frac{1}{R_{z}}\|w-z\| .
$$

Indeed, suppose that

$$
\left\|\left.\nabla \Phi_{k}\right|_{z}(w-z)\right\| \leq \frac{c}{R_{z}}\|w-z\|,
$$

for all $k=1, \ldots, d$. For $c$ small enough, this will lead to a contradiction. Let $w-z=\sum_{l} a_{l} p_{l}$. We have (using say Lemma 3.1.6)

$$
\left\|\left.\nabla \Phi_{k}\right|_{z}(w-z)\right\|=\left\|\left.\sum_{l} a_{l} \partial_{p_{l}} \Phi_{k}\right|_{z}\right\|=\left\|\left.\sum_{l \leq k} a_{l} \partial_{p_{l}} \Phi_{k}\right|_{z}\right\| \gtrsim\left(\left|a_{k}\right|-c \sum_{l<k}\left|a_{l}\right|\right) \frac{1}{R_{z}} .
$$

By induction, $\left|a_{k}\right| \leq \sum_{l=1}^{k} c^{l}\|w-z\|$. For $c$ small enough, $\left|a_{i}\right| \leq \frac{\|w-z\|}{d}$. This is a contradiction since $\left\|\sum_{i} a_{i} p_{i}\right\|=\|w-z\|$ and $\left\|p_{i}\right\|=1$.

We also have, by Proposition 3.4.1,

$$
\left\|\left.\nabla \Phi\right|_{w}-\left.\nabla \Phi\right|_{z}\right\|_{o p} \lesssim\left(\frac{\|z-w\|}{R_{z}}\right)^{\alpha} \frac{1}{R_{z}}
$$

Finally, by ensuring $\frac{\left\|z-w_{i}\right\|}{R_{z}}$ is smaller then a universal constant for $i=1,2$, we get from equation (3.1.15)

$$
\begin{aligned}
\left\|\Phi\left(w_{1}\right)-\Phi\left(w_{2}\right)\right\| & =\left|\int_{0}^{1} \nabla \Phi\right|_{t w_{1}+(1-t) w_{2}}\left(w_{1}-w_{2}\right) d t \mid \\
& =\left|\int_{0}^{1}\left(\left.\nabla \Phi\right|_{w_{1}}+\left(\left.\nabla \Phi\right|_{t w_{1}+(1-t) w_{2}}-\left.\nabla \Phi\right|_{w_{1}}\right)\right)\left(w_{1}-w_{2}\right) d t\right| \\
& \gtrsim \int_{0}^{1} \frac{1}{R_{z}}\left\|w_{1}-w_{2}\right\| d t \gtrsim \frac{1}{R_{z}} c_{0}\left\|w_{1}-w_{2}\right\|,
\end{aligned}
$$

which proves the lower bound (2.1.5). To prove the upper bound of (2.1.5), we observe that from Proposition 3.4.1 we have the upper bound

$$
\left|\gamma_{l} \partial_{p_{l}} \varphi_{i_{l}}(z)\right| \lesssim R_{z}^{-1}
$$

This completes the proof for the Euclidean case.

We now turn to the manifold case. Let $R_{z}$ be as in the Theorem. We take $c_{1} \leq \frac{1}{2} \delta_{0}$ chosen so that

$$
\left|g^{i l}(x)-\delta^{i l}\right|=\left|g^{i l}(x)-g^{i l}(z)\right|<\|g\|_{\alpha}\|x-z\|^{\alpha}<\epsilon_{0}
$$

for all $x \in B_{2 c_{1} R_{z}}(z)$. For this $g$, the above is carried on in local coordinates. It is then left to prove that the Euclidean distance in the range of the coordinate map is equivalent to the geodesic distance on the manifold. We have for all $x, y \in B_{c_{1} R_{z}}(z)$ $d_{\mathscr{M}}(x, y) \leq \int_{0}^{1}\left\|\frac{x-y}{\|x-y\|}\right\|_{g} d t \leq \int_{0}^{1}\left\|\frac{x-y}{\|x-y\|}\right\|_{\mathbf{R}^{d}}\left(1+\|g\|_{\alpha} t^{\alpha}\right) d t \lesssim \alpha\left(1+\|g\|_{\alpha}\right)\|x-y\|$. 
The converse can be proved as follows. Let $\xi:[0,1] \rightarrow \mathscr{M}$ be the geodesic from $x$ to $y$. $\xi$ is contained in $B_{2 d_{\mathscr{M}}(x, y)}(x)$ on the manifold, whose image in the local coordinate chart is contained in $B_{2\left(1+\|g\|_{\alpha}\right) d_{\mathscr{M}}(x, y)}(x)$. We have

$$
d_{\mathscr{M}}(x, y)=\int_{\xi}\|\dot{\xi}(t)\|_{g} \gtrsim\left(1-\|g\|_{\alpha}\right) \int_{\xi}\|\dot{\xi}(t)\|_{\mathbf{R}^{d}} \gtrsim\left(1-\|g\|_{\alpha}\right)\|x-y\| .
$$

\subsection{The case of $g \in \mathscr{C}^{\alpha}$.}

Proof of Theorems 2.1.1 and 2.2.1 for the case $g \in \mathscr{C}^{\alpha}$. We can now give a short proof for the $g \in \mathscr{C}^{\alpha}$ case, relying on the $\mathscr{C}^{2}$ case. We need the following Lemma, which we prove in Section 3.5.2.

Lemma 3.2.1. Let $J>0$ be given. If

$$
\left\|\tilde{g}_{n}^{i l}-g^{i l}\right\|_{L^{\infty}\left(B_{R}(z)\right)} \rightarrow{ }_{n} 0
$$

with $\left\|\tilde{g}_{n}^{i l}\right\|_{\mathscr{C}^{\alpha}}$ uniformly bounded and with fixed ellipticity constants (as in (2.2.1)), then for $j<J$

$$
\begin{gathered}
\left\|\varphi_{j}-\tilde{\varphi}_{j, n}\right\|_{L^{\infty}\left(B_{R}(z)\right)} \rightarrow_{n} 0, \\
\left\|\nabla\left(\varphi_{j}-\tilde{\varphi}_{j, n}\right)\right\|_{L^{\infty}\left(B_{R}(z)\right)} \rightarrow_{n} 0,
\end{gathered}
$$

and

$$
\left|\lambda_{j}-\tilde{\lambda}_{j, n}\right| \rightarrow_{n} 0
$$

Now, to conclude the proof of the Theorem for the $\mathscr{C}^{\alpha}$ case, let $J=c_{5}\left(d, \frac{1}{2} c_{\text {min }}\right.$, $\left.2 c_{\max },\|g\|_{\alpha}, \alpha\right) \cdot R_{z}^{-2}$. We may approximate $g$ in $\mathscr{C}^{\alpha}$ norm arbitrarily well by a $\mathscr{C}^{2}(\mathscr{M})$ metric. By the above Lemma, and the Theorem for the case of $\mathscr{C}^{2}$ metric, we obtain the Theorem for the $\mathscr{C}^{\alpha}$ case.

3.3. Heat kernels estimates. This section makes no assumptions on the finiteness of the volume of $\mathscr{M}$ and the existence of $C_{\text {count }}$.

3.3.1. Euclidean Dirichlet heat kernel estimates. We will start by proving the heat kernel estimates of Proposition 3.1.2 for the Dirichlet kernel $K^{\Omega}$. These estimates are in fact well known, and we include their proof here for completeness, and also to introduce in a simple setting the kind of probabilistic approach that will be used to obtain estimates in a more general context.

Proof of Proposition 3.1.2 for the Euclidean Dirichlet heat kernel. Let $B_{\omega}^{z}$ below be a Brownian path started at point $z \in \Omega$, and $\tau(\omega)$ its first hitting time of $\partial \Omega$. We recall that as a consequence of the Markov property we have (see e.g. [21], eqn. (9.5) p. 590)

$$
K_{t}^{\Omega}(z, w)=K_{t}^{\mathbf{R}^{d}}(z, w)-\mathbf{E}_{\omega}\left(K_{t-\tau(\omega)}^{\mathbf{R}^{d}}\left(B_{\omega}^{z}(\tau(\omega)), w\right) \chi_{t>\tau(\omega)}\right)
$$


Then,

$$
\begin{aligned}
& \mathbf{E}_{\omega}\left(K_{t-\tau(\omega)}^{\mathbf{R}^{d}}\left(B_{\omega}^{z}(\tau(\omega)(\omega)), w\right) \chi_{t>\tau(\omega)(\omega))}\right. \\
& =\mathbf{E}_{\omega}\left((4 \pi)^{\frac{-d}{2}}(t-\tau(\omega))^{\frac{-d}{2}} e^{\frac{-\|B(\tau(\omega))-w\|^{2}}{4(t-\tau(\omega))}} \chi_{t>\tau(\omega)}\right) \\
& \leq \mathbf{E}_{\omega}\left((4 \pi)^{\frac{-d}{2}}(t)^{\frac{-d}{2}} e^{\frac{-\|B(\tau(\omega))-w\|^{2}}{4 t}} \chi_{t>\tau(\omega))}\right. \\
& \leq \mathbf{E}_{\omega}\left((4 \pi)^{\frac{-d}{2}}(t)^{\frac{-d}{2}} e^{\frac{-\left(1-\delta_{0}\right)^{2} R_{z}^{2}}{4 t}} \chi_{t>\tau(\omega)}\right) \leq(4 \pi)^{\frac{-d}{2}}(t)^{\frac{-d}{2}} e^{\frac{-\left(1-\delta_{0}\right)^{2} R_{z}^{2}}{4 t}} \\
& \leq(4 \pi)^{\frac{-d}{2}}(t)^{\frac{-d}{2}} e^{\frac{-\left(1-\delta_{0}\right)^{2} R_{z}^{2}}{4\left(\delta_{1} R_{z}\right)^{2}}}=(4 \pi)^{\frac{-d}{2}}(t)^{\frac{-d}{2}} e^{\frac{-\left(1-\delta_{0}\right)^{2}}{4 \delta_{1}^{2}}},
\end{aligned}
$$

where for the first inequality we require $\frac{\|B(\tau)-w\|^{2}}{t}$ sufficiently large, which is implied by choosing $\delta_{0}<1$ and $\delta_{1}$ small enough. The last term can be made arbitrarily small by choosing $\delta_{1}$ small enough, independently of $\delta_{0}$ (as long as, say, $\delta_{0}<\frac{1}{2}$ ). We also have

$$
K_{t}^{\mathbf{R}^{d}}(z, w)=(4 \pi)^{\frac{-d}{2}}(t)^{\frac{-d}{2}} e^{\frac{-\|z-w\|^{2}}{4 t}} \leq(4 \pi)^{\frac{-d}{2}}(t)^{\frac{-d}{2}}
$$

and

$$
\begin{aligned}
K_{t}^{\mathbf{R}^{d}}(z, w) & =(4 \pi)^{\frac{-d}{2}}(t)^{\frac{-d}{2}} e^{\frac{-\|z-w\|^{2}}{4 t}} \geq(4 \pi)^{\frac{-d}{2}}(t)^{\frac{-d}{2}} e^{\frac{-\delta_{0}^{2} R_{z}^{2}}{4 t}} \\
& \geq(4 \pi)^{\frac{-d}{2}}(t)^{\frac{-d}{2}} e^{\frac{-\delta_{0}^{2}}{\delta_{1}^{2}}}
\end{aligned}
$$

If $\delta_{0}<\frac{1}{3}$, then $\frac{\left(1-\delta_{0}\right)^{2}}{4} \geq \delta_{0}$ and so by reducing $\delta_{1}$ (while fixing $\delta_{0}$ ) we can make the left-hand side of (3.3.2) arbitrarily small in comparison with (3.3.3). Now, from equation (3.3.1) we get (3.1.1) for the Dirichlet kernel.

Note that the range we have for $t$ and $\left\|B_{\omega}^{z}(\tau(\omega))-w\right\|$ imply that

$$
(4 \pi)^{\frac{-d}{2}}(t)^{\frac{-d}{2}} e^{\frac{-\left\|B_{\omega}^{z}(\tau(\omega))\right\|^{2}}{4 t}}
$$

is monotone increasing in $t$. Hence we also have

$$
\mathbf{E}_{\omega}\left(\chi_{s>\tau(\omega)} K_{s-\tau(\omega)}^{\mathbf{R}^{d}}\left(B_{\omega}^{z}(\tau(\omega)), w\right)\right) \leq(4 \pi)^{\frac{-d}{2}}(t)^{\frac{-d}{2}} e^{\frac{-\left(1-\delta_{0}\right)^{2}}{4 \delta_{1}^{2}}}
$$

If we also have $\frac{\delta_{0}}{\delta_{1}}$ is large enough, then $K_{s}^{\mathbf{R}^{d}}(z, w)$ is monotone increasing in $s$, and therefore

$$
K_{s}^{\mathbf{R}^{d}}(z, w) \leq K_{t}^{\mathbf{R}^{d}}(z, w) \leq(4 \pi)^{\frac{-d}{2}} t^{-\frac{d}{2}}
$$

For and fixed $\delta_{0}$, we may reduce $\delta_{1}$ so that by (3.3.4) is small, and thus we obtain the first part of (3.1.4) from (3.3.1). 
We now turn to estimates (3.1.2) and second and third parts of (3.1.4). We differentiate equation (3.3.1) and then we bound as follows:

$$
\begin{aligned}
& \left\|\nabla_{w} \mathbf{E}_{\omega}\left[\chi_{t>\tau(\omega)} K_{t-\tau(\omega)}^{\mathbf{R}^{d}}\left(B_{\omega}^{z}(\tau(\omega), w)\right)\right]\right\| \\
& =\| \nabla_{w} \mathbf{E}_{\omega}\left[\chi_{t>\tau(\omega)}(4 \pi)^{\frac{-d}{2}}(t-\tau(\omega))^{\frac{-d}{2}} e^{\left.\frac{-\left\|B_{\omega}^{z}(\tau(\omega))-w\right\|^{2}}{4(t-\tau(\omega))}\right] \|}\right. \\
& =\| \mathbf{E}_{\omega}\left[\chi_{t>\tau(\omega)} \nabla_{w}(4 \pi)^{\frac{-d}{2}}(t-\tau(\omega))^{\frac{-d}{2}} e^{\left.\frac{-\left\|B_{\omega}^{z}(\tau(\omega))-w\right\|^{2}}{4(t-\tau(\omega))}\right] \|}\right. \\
& =\left\|\mathbf{E}_{\omega}\left[\chi_{t>\tau(\omega)} \frac{B_{\omega}^{z}(\tau(\omega))-w}{2(t-\tau(\omega))}(4 \pi)^{\frac{-d}{2}}(t-\tau(\omega))^{\frac{-d}{2}} e^{\frac{-\left\|B_{\omega}^{z}(\tau(\omega))-w\right\|^{2}}{4(t-\tau(\omega))}}\right]\right\| \\
& \leq\left\|\mathbf{E}_{\omega}\left[\chi_{t>\tau(\omega)} \frac{\left\|B_{\omega}^{z}(\tau)-w\right\|}{2(t-\tau(\omega))}(4 \pi)^{\frac{-d}{2}}(t-\tau(\omega))^{\frac{-d}{2}} e^{\frac{-\left\|B_{\omega}^{z}(\tau(\omega))-w\right\|^{2}}{4(t-\tau)}}\right]\right\| \\
& \leq\left\|\mathbf{E}_{\omega}\left[\chi_{t>\tau(\omega)} \frac{\left\|B_{\omega}^{z}(\tau(\omega))-w\right\|}{2 t}(4 \pi)^{\frac{-d}{2}} t^{\frac{-d}{2}} e^{\frac{-\left\|B_{\omega}^{z}(\tau(\omega))-w\right\|^{2}}{4 t}}\right]\right\| \\
& =(4 \pi)^{\frac{-d}{2}} t^{\frac{-d-1}{2}} \mathbf{E}_{\omega}\left[\chi_{t>\tau(\omega)} \frac{\left\|B_{\omega}^{z}(\tau(\omega))-w\right\|}{2 \sqrt{t}} e^{\frac{-\left\|B_{\omega}^{z}(\tau(\omega))-w\right\|^{2}}{4 t}}\right] \\
& \leq(4 \pi)^{\frac{-d}{2}} t^{\frac{-d-1}{2}} \mathbf{E}_{\omega}\left[\chi_{t>\tau(\omega)} \frac{1-\delta_{0}}{2 \delta_{1}} e^{\frac{-\left(1-\delta_{0}\right)^{2}}{4 \delta_{1}^{2}}}\right] \\
& =(4 \pi)^{\frac{-d}{2}} t^{\frac{-d-1}{2}} \frac{1-\delta_{0}}{2 \delta_{1}} e^{\frac{-\left(1-\delta_{0}\right)^{2}}{4 \delta_{1}^{2}}}=: C\left(\delta_{0}, \delta_{1}\right),
\end{aligned}
$$

where for the second equality we use the dominated convergence theorem, for the inequalities in the fifth and in the penultimate line we choose $\delta_{0}<1$ and $\delta_{1}$ small enough. Note that $\delta_{1} \rightarrow 0$ implies that $C\left(\delta_{0}, \delta_{1}\right) \rightarrow 0$. Observe that these estimates hold also with $\nabla_{w}$ replaced by $\frac{\partial}{\partial p}$.

We also have

$$
\begin{aligned}
\nabla_{w} K_{t}^{\mathbf{R}^{d}}(z, w) & =\nabla_{w}(4 \pi)^{\frac{-d}{2}} t^{\frac{-d}{2}} e^{\frac{-\|z-w\|^{2}}{4 t}}=(4 \pi)^{\frac{-d}{2}} t^{\frac{-d-1}{2}} \frac{(z-w)}{2 \sqrt{t}} e^{\frac{-\|z-w\|^{2}}{4 t}} \\
& \geq(4 \pi)^{\frac{-d}{2}} t^{\frac{-d-1}{2}} \frac{\delta_{0} R_{z}}{t} e^{\frac{-\delta_{0}^{2}}{\delta_{1}^{2}}}(z-w)
\end{aligned}
$$

(with inequality understood entrywise) where as above the last inequality holds for $\delta_{0}<1$ and $\delta_{1}$ small enough. If $p$ is parallel to $z-w$, the same estimates hold if we replace $\nabla_{w}$ by $\partial_{p}$. Hence, for any fixed $\delta_{0}$, by reducing $\delta_{1}$ we get

$$
\left\|\nabla_{w} K_{t}^{\Omega}(z, w)-\nabla_{w} K_{t}^{\mathbf{R}^{d}}(z, w)\right\| \leq(4 \pi)^{\frac{-d}{2}} \frac{R_{z}}{2 t} t^{\frac{-d}{2}} e^{\frac{-\left(1-\delta_{0}\right)^{2}}{4 \delta_{1}^{2}}}
$$

and therefore

$$
\left\|\nabla_{w} K_{t}^{\Omega}(z, w)\right\| \sim\left\|\nabla_{w} K_{t}^{\mathbf{R}^{d}}(z, w)\right\| \sim t^{\frac{-d}{2}} \frac{R_{z}}{t} .
$$

The estimate (3.1.2) involving $\partial_{p}$ is proven analogously. The second and third parts of (3.1.4) follow as above. Finally, to prove (3.1.3) we use (3.3.5) to obtain

$$
\left\|\partial_{q} K_{t}^{\Omega}(z, w)-\partial_{q} K_{t}^{\mathbf{R}^{d}}(z, w)\right\| \leq(4 \pi)^{\frac{-d}{2}} \frac{R_{z}}{2 t} t^{\frac{-d}{2}} e^{\frac{-\left(1-\delta_{0}\right)^{2}}{4 \delta_{1}^{2}}} \leq C_{9}\left(\delta_{0}, \delta_{1}\right) t^{\frac{-d}{2}} \frac{R_{z}}{t} .
$$


3.3.2. Local and global heat kernels. In this section, let $K$ be the heat kernel, Dirichlet or Neumann, for

(i) a domain $\Omega$ (possibly unbounded), and a uniformly elliptic operator $\Delta$ as in (2.2.3), with $g \in \mathscr{C}^{2}(\Omega)$

(ii) a manifold $\mathscr{M}$ with $g \in \mathscr{C}^{2}$ satisfying the requirements in Section 2.2, and let $\Delta$ be the associated Laplacian.

Remark 3.3.1. We emphasize that in this section we do not assume that the volume of $\mathscr{M}$ is finite.

Observe that in both settings the existence of an associated Brownian motion is guaranteed ([37] for the $\mathbf{R}^{d}$ case and the manifold case then follows from uniqueness). The following result connects $K$ with the Dirichlet kernel on a ball, associated with $\Delta$, to which the estimates of the previous section apply: this will allow us to extend estimates for the Dirichlet heat kernel on a ball to the general heat kernel $K$. A more detailed account of the ideas in the following proposition appears in Stroock's recent book [48] (Section 5.2 Duhamel's Formula).

Proposition 3.3.2. Let $z \in \Omega$ and $r \leq \operatorname{dist}(z, \partial \Omega)$, or $z \in \mathscr{M}$ and $r \leq r_{U}(z)$. Let $x, y \in B\left(z, \frac{1}{4} r\right)$. For each path $B_{\omega}^{x}$ (starting at $x$ ), we define $\tau_{1}(\omega) \leq \tau_{2}(\omega) \leq \ldots$ as follows. Let $\tau_{1}(\omega)$ be the first time that $B_{\omega}^{x}$ re-enters $B\left(x, \frac{3}{8} r\right)$ after having exited $B\left(x, \frac{1}{2} r\right)$ (if this does not happen, let $\left.\tau_{1}(\omega)=+\infty\right)$. Let $x_{1}=B_{\omega}^{x}\left(\tau_{1}\right)$. By induction, for $n>1$ let $\tau_{n}(\omega)$ be the first time after $\tau_{n-1}(\omega)$ that $B_{\omega}$ re-enters $B\left(x, \frac{3}{8} r\right)$ after having exited $B\left(x, \frac{1}{2} r\right)$, or $+\infty$ otherwise. Let $x_{n}=B_{\omega}^{x}\left(\tau_{n}\right)$. If $\tau_{n}(\omega)=+\infty$, let $\tau_{n+k}(\omega)=+\infty$ for all $k \geq 0$. Then

$$
K_{s}(x, y)=K_{s}^{D}(x, y)+\sum_{n=1}^{\infty} \mathbf{E}_{\omega}\left[K_{s-\tau_{n}(\omega)}^{D}\left(x_{n}(\omega), y\right) \mid \tau_{n}<s\right] P\left(\tau_{n}<s\right),
$$

where

$$
K_{s}^{D}=K_{s}^{\operatorname{Dir}\left(B_{\frac{1}{2} r}(x)\right)}
$$

is the heat kernel at time $s$ for the ball $B\left(x, \frac{1}{2} r\right)$ with Dirichlet boundary conditions. Moreover, there exists an $M=M\left(c_{\min }, c_{\max }\right)$ such that

$$
P\left(\tau_{n}<s\right) \lesssim_{d, c_{\min }, c_{\max }} \exp \left\{-n \frac{r^{2}}{M s}\right\}
$$

Remark 3.3.3. In our applications of this proposition, we have $r \sim \delta_{0} R_{z}$. In that case, if $s^{\frac{1}{2}}<\delta_{1} R_{z}$, for $\frac{\delta_{0}}{\delta_{1}}$ sufficiently large (depending only, on $d, c_{\min }, c_{\max }$ ), the factor $\exp \left\{-n \frac{r^{2}}{M s}\right\}$ can be made arbitrarily small. This gives us control (exponential in $n$ ) on the right-hand side of (3.3.7). Hence, for any fixed $\delta_{0}$, for $\delta_{1} \rightarrow 0$ the right-hand-side of equation (3.3.6) is dominated by the first summand.

Proof of Proposition 3.3.2. The proofs for the case of a domain $\Omega$ and the case of a manifold $\mathscr{M}$ are identical. We have, for any fixed, small enough, $\epsilon>0$,

$$
\begin{aligned}
P^{\Omega}\left(B_{\omega}^{x}(s) \in\right. & \left.B_{\epsilon}(y)\right)=P^{\Omega}\left(B_{\omega}^{x}(s) \in B_{\epsilon}(y), \tau_{1} \geq s\right)+P^{\Omega}\left(B_{\omega}^{x}(s) \in B_{\epsilon}(y), \tau_{1}<s\right) \\
= & \int_{B_{\epsilon}(y)} K_{s}^{D}\left(x, y^{\prime}\right) d y^{\prime}+P^{\Omega}\left(B_{\omega}^{x}(s) \in B_{\epsilon}(y), \tau_{2} \geq s \mid \tau_{1}<s\right) P\left(\tau_{1}<s\right) \\
& +P^{\Omega}\left(B_{\omega}^{x_{1}}(s) \in B_{\epsilon}(y), \tau_{1}<s, \tau_{2}<s\right)
\end{aligned}
$$




$$
\begin{aligned}
= & \int_{B_{\epsilon}(y)} K_{s}^{D}\left(x, y^{\prime}\right) d y^{\prime}+P^{\Omega}\left(B_{\omega}^{x_{1}}\left(s-\tau_{1}\right) \in B_{\epsilon}(y), \tau_{2} \geq s \mid \tau_{1}<s\right) P\left(\tau_{1}<s\right) \\
& +P^{\Omega}\left(B_{\omega}^{x_{1}}(s) \in B_{\epsilon}(y), \tau_{1}<s, \tau_{2}<s\right) \\
= & \int_{B_{\epsilon}(y)} K_{s}^{D}\left(x, y^{\prime}\right) d y^{\prime}+\int_{B_{\epsilon}(y)} \mathbf{E}_{\omega}\left[K_{s-\tau_{1}}^{D}\left(x_{1}, y^{\prime}\right) \mid \tau_{1}<s\right] d y P\left(\tau_{1}<s\right) \\
& +P^{\Omega}\left(B_{\omega}^{x_{1}}(s) \in B_{\epsilon}(y), \tau_{2}<s\right) \\
= & \cdots \\
= & \int_{B_{\epsilon}(y)} K_{s}^{D}\left(x, y^{\prime}\right) d y^{\prime}+\sum_{n=1}^{+\infty} \int_{B_{\epsilon}(y)} \mathbf{E}_{\omega}\left[K_{s-\tau_{n}}^{D}\left(x_{i}, y^{\prime}\right) \mid \tau_{n}<s\right] d y^{\prime} P\left(\tau_{n}<s\right) .
\end{aligned}
$$

By dividing by $|B(y, \epsilon)|$ and taking the limit as $\epsilon \rightarrow 0^{+}$, we obtain (3.3.6).

In order to estimate $P\left(\tau_{n}<s\right)$, we need the following

Lemma 3.3.4. Let $\Omega$ be a domain corresponding to a uniformly elliptic operator as in (2.2.3). Let $\tau$ be the first exit time from $B_{R}(z) \subseteq \Omega$ for the corresponding stochastic process started at $z$. Then there exists $M=M\left(d, c_{\min }, c_{\max }\right)>0$ such that

$$
P^{z}(\{\tau \leq s\}) \lesssim{d, c_{\min }, c_{\max }} \exp \left\{-R^{2}(2 M s)^{-1}\right\} .
$$

Similarly for $z \in \mathscr{M}$ and $R \leq r_{U}(z)$.

Proof. First note that without loss of generality we may replace $\Omega$ by $B_{2 R}(z)$ with Dirichlet boundary conditions, and then replace $B_{2 R}(z)$ by $\mathbf{R}^{d}$ by extending the coefficients $g^{i j}$ to $\tilde{g}^{i j}$ defined on all of $\mathbf{R}^{d}$. Let $\hat{K}$ be the associated heat kernel. For any $s^{\prime}>0$ and $x, y \in B_{R}(z)$

$$
s^{\prime-\frac{d}{2}} \exp \left\{-\frac{|x-y|^{2}}{A_{1} s^{\prime}}\right\} \lesssim_{c_{\min }, c_{\max }, d} \hat{K}_{s^{\prime}}(x, y) \lesssim_{d, c_{\min }, c_{\max }} s^{\prime-\frac{d}{2}} \exp \left\{-\frac{|x-y|^{2}}{A_{2} s^{\prime}}\right\} .
$$

holds for $M=M\left(c_{\min }, c_{\max }, d\right)$ and $A_{i}=A_{i}\left(c_{\min }, c_{\max }, d\right)$ (see [16], Corollary 3.2.8 and Theorem 3.3.4). We now follow a short proof by Stroock [49]. By the strong Markov property, we have

$$
P^{\Omega}\left(B_{\omega}^{z}(s) \notin B_{R}(z)\right)=\mathbf{E}_{\omega}^{z}\left[P\left(B_{\omega}^{\omega(\tau(\omega))}(s-\tau(\omega)) \notin B_{R}(z)\right) \chi_{\{\tau(\omega)<s\}}\right] .
$$

From the lower bound in equation (3.3.9) we have that if $x \in \partial B_{R}(z)$ and $s>0$ then $P\left(B_{\omega}^{x}(s) \notin B_{R}(x)\right) \geq \epsilon\left(c_{\min }, c_{\max }, d\right)$. Combining this with the upper bound in equation (3.3.9) we have

$$
P^{z}(\tau \leq s) \leq \epsilon\left(d, c_{\min }, c_{\max }\right)^{-1} P\left(B_{\omega}^{z}(s) \notin B_{R}(z)\right) \lesssim_{d, c_{\min }, c_{\max }} \exp \left\{-R^{2}(2 M s)^{-1}\right\} .
$$

We go back to the proof of Proposition 3.3.2. To estimate $P\left(\tau_{n}<s \mid \tau_{n-1}<\right.$ $\left.s, \ldots, \tau_{1}<s\right)$, we observe that between $\tau_{n-1}$ and $\tau_{n}$, the path $\omega$ has to cross both $\partial B_{\frac{3}{8} r}(z)$ and $\partial B_{\frac{1}{2} r}(z)$ : let $\tau_{n}^{*}$ and $\tau_{n}^{* *}$ be the first time this happens, and let $y^{*}=$ $\omega\left(\tau_{n}^{*}\right)$. Then

$$
\begin{aligned}
P\left(\tau_{n}<s \mid\right. & \left.\tau_{n-1}<s, \ldots, \tau_{1}<s\right) \leq P\left(\tau_{n}^{* *}-\tau_{n}^{*}<s\right) \\
\leq & \sup _{y^{*} \in B_{\frac{3}{8} r}(z)} P^{y^{*}}\left(\sup _{s^{\prime} \in[0, s]}\left\|B_{\omega}\left(s^{\prime}\right)-y^{*}\right\|>\frac{1}{8} r\right) \lesssim_{c_{\min }, c_{\max }, d} e^{-\frac{\left(\frac{1}{8} r\right)^{2}}{2 M s}} .
\end{aligned}
$$


Therefore we have

$$
\begin{aligned}
P\left(\tau_{n}<s\right) & =P\left(\tau_{1}<s, \tau_{2}<s, \ldots, \tau_{n}<s\right) \\
& =\left(\prod_{l=2}^{n} P\left(\tau_{l}<s \mid \tau_{l-1}<s, \ldots, \tau_{1}<s\right)\right) P\left(\tau_{1}<s\right) \\
& \lesssim_{c_{\min }, c_{\max }, d} \exp \left\{-n\left(\frac{1}{8} r\right)^{2}(2 M s)^{-1}\right\} .
\end{aligned}
$$

Renaming $128 M$ to $M$ we get the lemma.

Remark 3.3.5. As it is clear from the proof, the proposition holds for any boundary condition on a manifold or domain.

3.3.3. Euclidean Neumann heat kernel estimates. We use the results of the previous two sections to prove the Neumann case of Proposition 3.1.2:

Proof of Proposition 3.1.2 for the Euclidean Neumann heat kernel. The starting point is Proposition 3.3.2, which allows us to localize. We use Proposition 3.1.2 for the case of $B_{2 \delta_{0} R_{z}}(z)$. For this proof, we denote by $C_{2}[B]$ be the $C_{2}$ constant for the Dirichlet ball case. For $s \leq t$ we have using equation (3.3.10),

$$
\begin{aligned}
\left|K_{s}(x, y)-K_{s}^{2 \delta_{0} R_{z}}(x, y)\right| & =\left|\sum_{n=1}^{+\infty} \mathbf{E}_{\omega}\left[K_{s-\tau_{n}}^{2 \delta_{0} R_{z}}\left(x_{n}(\omega), y\right) \mid \tau_{n}<s\right] P_{\omega}\left(\tau_{n}<s\right)\right| \\
& \lesssim C_{2}[B] \sum_{n=1}^{\infty} t^{-\frac{d}{2}} \underbrace{\exp \left\{-n\left(\frac{\delta_{0} R_{z}}{2}\right)^{2}(M s)^{-1}\right\}}_{\text {equation }(3.3 .10)} \\
& \lesssim C_{2}[B], \delta_{0}, \delta_{1} t^{-\frac{d}{2}} \exp \left\{-\left(\frac{\delta_{0} R_{z}}{2}\right)^{2}(M s)^{-1}\right\} .
\end{aligned}
$$

This proves (3.1.1) and the first part of (3.1.4) (see Remark 3.3.3). For the gradient estimates, i.e. (3.1.2), (3.1.3), and the second and third part of (3.1.4),

$$
\begin{aligned}
\left\|\nabla_{y} K_{s}(x, y)-\nabla_{y} K_{s}^{2 \delta_{0} R_{z}}(x, y)\right\| & \leq \sum_{n=1}^{+\infty}\left\|\nabla_{y} \mathbf{E}_{\omega}\left[K_{s-\tau_{n}}^{2 \delta_{0} R_{z}}\left(x_{n}(\omega), y\right) \mid \tau_{n}<s\right]\right\| P_{\omega}\left(\tau_{n}<s\right) \\
& \lesssim_{C_{2}^{\prime}[B]} \sum_{n=1}^{\infty} t^{-\frac{d}{2}} \frac{\delta_{0} R_{z}}{t} \underbrace{\exp \left\{-n\left(\frac{\delta_{0} R_{z}}{2}\right)^{2}(M s)^{-1}\right\}}_{\text {equation }(3.3 .10)} \\
& \lesssim_{C_{2}^{\prime}[B], \delta_{0}, \delta_{1}} t^{-\frac{d}{2}} \frac{\delta_{0} R_{z}}{t} \exp \left\{\left(\frac{\delta_{0} R_{z}}{2}\right)^{2}(M s)^{-1}\right\}
\end{aligned}
$$

giving us $C_{9}$. By Remark 3.3.3 the exponential term from equation (3.3.10) can be made small enough so that we obtain estimate (3.1.2) as well as the second and third parts of (3.1.4).

The proof for the manifold case is postponed to Section 3.5.3.

\subsection{Heat kernel and eigenfunctions.}

3.4.1. Bounds on eigenfunctions. We record some inequalities that will be used in what follows. 
Proposition 3.4.1. Assume $g \in \mathscr{C}^{\alpha}$. There exists $b_{1}<1$, and $C_{P}>0$ that depends on $d, c_{\min }, c_{\max },\|g\|_{\alpha}, \alpha$ such that for any eigenfunction $\varphi_{j}$ of $\Delta_{\mathscr{M}}$ on $B_{R}(z)$, corresponding to the eigenvalue $\lambda_{j}$, and $R \leq R_{z}$, the following estimates hold. For $w \in B_{b_{1} R}(z)$ and $x, y \in B_{b_{1} R}(z)$,

$$
\begin{gathered}
\left|\varphi_{j}(w)\right| \leq C_{P} P_{1}\left(\lambda_{j} R^{2}\right)\left(f_{B_{R}(z)}\left|\varphi_{j}\right|^{2}\right)^{\frac{1}{2}}, \\
\left\|\nabla \varphi_{j}(w)\right\| \leq C_{P} \frac{P_{2}\left(\lambda_{j} R^{2}\right)}{R}\left(f_{B_{R}(z)}\left|\varphi_{j}\right|^{2}\right)^{\frac{1}{2}}, \\
\left\|\nabla \varphi_{j}(x)-\nabla \varphi_{j}(y)\right\| \leq C_{P} \frac{P_{3}\left(\lambda_{j} R^{2}\right)}{R^{1+\alpha}}\left(f_{B_{R}(z)}\left|\varphi_{j}\right|^{2}\right)^{\frac{1}{2}}\|x-y\|^{\alpha},
\end{gathered}
$$

where $P_{1}(x)=(1+x)^{\frac{1}{2}+\beta}, P_{2}(x)=(1+x)^{\frac{3}{2}+\beta}, P_{3}(x)=(1+x)^{\frac{5}{2}+\beta}$, with $\beta$ the smallest integer larger than or equal to $\frac{d-2}{4}$.

We postpone the proof to Section 3.5.2. Related estimates can be found in $[15,46,45,44,56]$ and references therein.

3.4.2. Truncated heat kernel and selecting eigenfunctions. The goal of this section is to prove Lemma 3.1.4 and 3.1.5. All the results of this section and their proofs will be independent on whether we talk about the Dirichlet or Neumann heat kernel, and on whether we talk about the standard Laplacian or about a uniformly elliptic operator satisfying our usual assumptions and whether we talk about a manifold $\mathscr{M}$ or a domain $\Omega$. This is because the only tools we will need to obtain the results in this section are the spectral expansion of the heat kernel (3.1.5), the elliptic estimates of Proposition 3.4.1, the assumption on $C_{\text {count }}(2.1 .2)$, and the bound

$$
K_{t}(z, w) \leq K_{t}(z, z)^{\frac{1}{2}} K_{t}(w, w)^{\frac{1}{2}}
$$

which is a straightforward application of Cauchy-Schwartz inequality to (3.1.5).

Proof of Lemma 3.1.4. We upper bound the tail of the heat kernel:

$$
\begin{aligned}
\left|\sum_{\lambda_{j} \geq A t^{-1}} \varphi_{j}(z) \varphi_{j}(w) e^{-\lambda_{j} t}\right| & \leq e^{\frac{-A}{2}}\left|\sum_{\lambda_{j} \geq A t^{-1}} \varphi_{j}(z) \varphi_{j}(w) e^{\frac{-\lambda_{j} t}{2}}\right| \\
& \leq e^{\frac{-A}{2}}\left|K_{\frac{t}{2}}(z, z)\right|^{\frac{1}{2}}\left|K_{\frac{t}{2}}(w, w)\right|^{\frac{1}{2}} \lesssim_{C_{2}} t^{\frac{-d}{2}} e^{\frac{-A}{2}}
\end{aligned}
$$

by (3.4.4) and Proposition 3.1.2. For $A$ large enough, this implies (3.1.7).

For the gradient, we use Proposition 3.4.1, and observe that $x^{n} e^{\frac{-x}{4}}$ is a decreasing function if $x$ is large enough. We let $r_{w}=\left(1-\delta_{0}\right) R_{z}$. Then

$$
\begin{aligned}
\left\|\sum_{\lambda_{j} \geq A t^{-1}} \varphi_{j}(z) \nabla \varphi_{j}(w) e^{-\lambda_{j} t}\right\| & \leq\left\|e^{\frac{-A}{2}} \sum_{\lambda_{j} \geq A t^{-1}} \varphi_{j}(z) \nabla \varphi_{j}(w) e^{\frac{-\lambda_{j} t}{2}}\right\| \\
& \leq e^{\frac{-A}{2}}\left|K_{\frac{t}{2}}(z, z)\right|^{\frac{1}{2}}\left(\sum_{\lambda_{j} \geq A t^{-1}}\left\|\nabla \varphi_{j}(w)\right\|^{2} e^{\frac{-\lambda_{j} t}{2}}\right)^{\frac{1}{2}}
\end{aligned}
$$




$$
\begin{aligned}
& \lesssim_{C_{p}} e^{\frac{-A}{2}}\left|K_{\frac{t}{2}}(z, z)\right|^{\frac{1}{2}}\left(\sum_{\lambda_{j} \geq A t^{-1}} P_{2}\left(\lambda_{j} r_{w}^{2}\right)^{2} r_{w}^{-2} f_{B\left(w, \frac{1}{2} r_{w}\right)}\left|\varphi_{j}\right|^{2} e^{\frac{-\lambda_{j} t}{2}}\right)^{\frac{1}{2}} \\
& \lesssim_{C_{P}, \delta_{1}} e^{\frac{-A}{2}}\left|K_{\frac{t}{2}}(z, z)\right|^{\frac{1}{2}}\left(\sum_{\lambda_{j} \geq A t^{-1}} f_{B\left(w, \frac{1}{2} r_{w}\right)}\left|\varphi_{j}\right|^{2} e^{\frac{-\lambda_{j} t}{2}} P_{2}\left(\lambda_{j} t\right)^{2} \frac{1}{t} e^{\frac{-\lambda_{j} t}{2}}\right)^{\frac{1}{2}} \\
& \lesssim_{C_{P}, \delta_{1}} e^{\frac{-A}{2}}\left|K_{\frac{t}{2}}(z, z)\right|^{\frac{1}{2}}\left(\sum_{\lambda_{j} \geq A t^{-1}} f_{B\left(w, \frac{1}{2} r_{w}\right)}\left|\varphi_{j}\right|^{2} e^{\frac{-\lambda_{j} t}{4}} \frac{1}{t}\right)^{\frac{1}{2}} \\
& \lesssim_{C_{P}, \delta_{1}} e^{\frac{-A}{2}}\left|K_{\frac{t}{2}}(z, z)\right|^{\frac{1}{2}}\left(f_{B\left(w, \frac{1}{2} r_{w}\right)} K_{\frac{t}{4}}\left(w^{\prime}, w^{\prime}\right) d w^{\prime} \frac{1}{t}\right)^{\frac{1}{2}} \\
& \lesssim_{C_{P}, \delta_{1}, C_{2}} e^{\frac{-A}{2}} t^{\frac{-d}{2}} \frac{1}{\sqrt{t}} .
\end{aligned}
$$

Now we consider the contribution of the low frequency eigenfunctions to the gradient. Proceeding as above, and recalling that in this case $\lambda_{j} r_{w}^{2} \leq \frac{A^{\prime}}{\delta_{1}}$, we obtain

$$
\begin{aligned}
\| & \sum_{\lambda_{j} \leq A^{\prime} t^{-1}} \varphi_{j}(z) \nabla \varphi_{j}(w) e^{-\lambda_{j} t} \| \\
& \lesssim_{C_{P}}\left|K_{t}(z, z)\right|^{\frac{1}{2}}\left(\sum_{\lambda_{j} \leq A^{\prime} t^{-1}} P_{2}\left(\lambda_{j} r_{w}^{2}\right)^{2} r_{w}^{-2} f_{B\left(w, \frac{1}{2} r_{w}\right)}\left|\varphi_{j}\right|^{2} e^{-\lambda_{j} t}\right)^{\frac{1}{2}} \\
& \lesssim_{C_{P}} P_{2}\left(\frac{A^{\prime}}{\delta_{1}}\right)\left|K_{t}(z, z)\right|^{\frac{1}{2}} \frac{1}{r_{w}}\left(\sum_{\lambda_{j} \leq A^{\prime} t^{-1}} f_{B\left(w, \frac{1}{2} r_{w}\right)}\left|\varphi_{j}\right|^{2} e^{-\lambda_{j} t}\right)^{\frac{1}{2}} \\
& \lesssim_{C_{P}} P_{2}\left(\frac{A^{\prime}}{\delta_{1}}\right)\left|K_{t}(z, z)\right|^{\frac{1}{2}} \frac{1}{r_{w}}\left(\sum_{\lambda_{j} \leq A^{\prime} t^{-1}} f_{B\left(w, \frac{1}{2} r_{w}\right)}\left|\varphi_{j}\right|^{2} e^{-\frac{\lambda_{j} t}{A^{\prime}}}\right)^{\frac{1}{2}} \\
& \lesssim_{C_{P}, C_{2}} P_{2}\left(\frac{A^{\prime}}{\delta_{1}}\right) \frac{1}{r_{w}}\left|K_{t}(z, z)\right|^{\frac{1}{2}}\left(\int_{B\left(w, \frac{1}{2} r_{w}\right)} K_{t / A^{\prime}}\left(w^{\prime}, w^{\prime}\right)\right)^{\frac{1}{2}} \\
& \lesssim_{C_{P}, C_{2}} P_{2}\left(\frac{A^{\prime}}{\delta_{1}}\right) \frac{1}{r_{w}} t^{-\frac{d}{2}} A^{\prime \frac{d}{4}} .
\end{aligned}
$$

Thus, by reducing $A^{\prime}$ we get the bound (3.1.8) and (3.1.9). (We note that an alternative approach to the introduction of $e^{-\frac{\lambda_{j} t}{A^{\prime}}}$ would have been to reduce $\delta_{1}$ and note that $\frac{r_{w}}{t}$ is as large as we want in comparison with $\frac{1}{r_{w}}$, and then to reduce $A^{\prime}$ to compensate for the reduction in $\delta_{1}$.)

For a domain with Dirichlet boundary conditions, we automatically have a bound on $C_{\text {count }}$ as in (2.1.2): 
Lemma 3.4.2. (Weyl's Lemma for Dirichlet boundary conditions) Let $\Omega$ be a domain in $\mathbf{R}^{d}, \Delta$ a uniformly elliptic operator on $\Omega$ as in (2.2.1), with Dirichlet boundary conditions. Let $\lambda_{0} \leq \lambda_{1} \leq \ldots$ be the eigenvalues of $\Delta$. Then

$$
\#\left\{j: \lambda_{j} \leq \lambda\right\} \quad \lesssim_{d, c_{\min }, c_{\max }}|\Omega| \lambda^{\frac{d}{2}}
$$

Proof. Let $K^{\Omega}$ the associated heat kernel. Extend the coefficient $g^{i j}$ to $\mathbf{R}^{d} \backslash \Omega$ by letting $g^{i j}=\delta^{i j}$, and let $\tilde{K}$ be the associated heat kernel on $\mathbf{R}^{d}$. Then $K^{\Omega}$ is pointwise dominated by $\tilde{K}$. Then by estimate (3.3.9) we have, following [23]:

$$
\begin{aligned}
\#\left\{j: \lambda_{j} \leq \lambda\right\} & \leq e \cdot \sum e^{\frac{-\lambda_{j}}{\lambda}}=e \cdot \int_{\Omega} K_{\frac{1}{\lambda}}^{\Omega}(x, x) d x \\
& \leq e \cdot \int_{\Omega} \tilde{K}_{\frac{1}{\lambda}}(x, x) d x \lesssim_{d, c_{\min }, c_{\max }}|\Omega| \lambda^{\frac{d}{2}} .
\end{aligned}
$$

Remark 3.4.3. Notice that in the Dirichlet case $C_{\text {count }}$ is independent of $\Omega$. In the Neumann case, if one has good enough estimates on the trace of the corresponding heat kernel, the same proof applies. In general these estimates will depend on $\Omega$, and $C_{\text {count }}$ will not be independent of $\Omega$ (see e.g. [26, 34]).

Proof of Lemma 3.1.5. In view of Lemma 3.1.4, we will show that the terms in the eigenfunction series corresponding to $j \in \Lambda_{L}(A) \cap \Lambda_{H}\left(A^{\prime}\right)$ but $j \notin \Lambda_{E}\left(p, z, R_{z}, \delta_{0}, c_{0}\right)$ do not contribute significantly to the lefthand side of (3.1.11). Let $\Lambda_{1}=\Lambda_{L}(A) \cap$ $\Lambda_{H}\left(A^{\prime}\right) \cap\left(\Lambda_{E}\left(p, z, R_{z}, \delta_{0}, c_{0}\right)\right)^{c}$. We thus have

$$
\begin{aligned}
\left|\sum_{\lambda_{j} \in \Lambda_{1}} \varphi_{j}(w) \partial_{p} \varphi_{j}(z) e^{-\lambda_{j} t}\right| & \leq \sum_{\lambda_{j} \in \Lambda_{1}}\left|\varphi_{j}(w)\right|\left|\partial_{p} \varphi_{j}(z) e^{-\lambda_{j} t}\right| \\
& \leq\left(\sum_{\lambda_{j} \in \Lambda_{1}}\left|\varphi_{j}(w)\right|^{2} e^{-\lambda_{j} t}\right)^{\frac{1}{2}}\left(\sum_{\lambda_{j} \in \Lambda_{1}}\left|\partial_{p} \varphi_{j}(z)\right|^{2} e^{-\lambda_{j} t}\right)^{\frac{1}{2}} \\
& \left.\leq K_{t}(w, w)^{\frac{1}{2}}\left(\sum_{\lambda_{j} \in \Lambda_{1}}\left|\partial_{p} \varphi_{j}(z)\right|^{2} e^{-\lambda_{j} t}\right)^{\frac{1}{2}}\right)^{\frac{1}{2}} \\
& \leq K_{t}(w, w)^{\frac{1}{2}} c_{0} \frac{1}{R}\left(\sum_{\lambda_{j} \in \Lambda_{1}} \underset{B\left(z, \frac{1}{2} \delta_{0} R_{z}\right)}{f}\left|\varphi_{j}\right|^{2} e^{-\lambda_{j} t}\right)^{f} K_{t}\left(z^{\prime}, z^{\prime}\right)^{\frac{1}{2}} d z^{\prime} . \\
& \leq c_{0} \frac{1}{R_{z}} K_{t}(w, w)^{\frac{1}{2}} \underset{z^{\prime} \in\left(z, \frac{1}{2} \delta_{0} R_{z}\right)}{f}
\end{aligned}
$$

Hence by reducing $c_{0}$ and using Proposition 3.1.2 together with Lemma 3.1.4 we conclude the proof. We note that the constant $C_{9}$ comes into play since we have to estimate the left hand side of equation (3.1.11).

Remark 3.4.4. The following proof is the only place where we use the bound on $C_{\text {count }}(2.1 .2)$. 
Proof of Lemma 3.1.6. For sufficiently small $b$, Equations (3.4.2) and (3.4.3) from Proposition 3.4.1, together with the definition of $\Lambda_{E}\left(p, z, R_{z}, \delta_{0}, c_{0}\right)$ give equation (3.1.13). We turn to showing equation (3.1.14), which is where $C_{\text {count }}$ will appear.

Since at this point all the constants are fixed, to ease the notation we let $\Lambda:=$ $\Lambda_{L}(A) \cap \Lambda_{H}\left(A^{\prime}\right) \cap \Lambda_{E}\left(p, z, R_{z}, \delta_{0}, c_{0}\right)$. Let $w \in B\left(z, \delta_{0} R_{z}\right) \backslash B\left(z, \frac{1}{2} \delta_{0} R_{z}\right)$ with $w-z$ in the direciton of p. Observe that Proposition 3.1.2 and Lemma 3.1.5 imply that

$$
\begin{aligned}
& K_{\frac{t}{2}}(w, w) \frac{R_{z}}{t} \sim_{C_{1}}^{C_{2}} t^{\frac{-d}{2}} \frac{R_{z}}{t} \sim_{C_{1}^{\prime}}^{C_{2}^{\prime}}\left|\partial_{p} K_{t}(w, z)\right| \sim_{C_{5}}^{C_{6}}\left|\sum_{\lambda_{j} \in \Lambda} \varphi_{j}(w) \partial_{p} \varphi_{j}(z) e^{-\lambda_{j} t}\right| \\
& \lesssim_{\left\{d, c_{\min }, c_{\max },\|g\|_{\alpha}, \alpha\right\}} R_{z}^{-1} \sum_{\lambda_{j} \in \Lambda}\left|\varphi_{j}(w) e^{-\lambda_{j} t}\right|\left(f_{B\left(z, \frac{1}{2} \delta_{0} R_{z}\right)}\left|\varphi_{j}\right|^{2}\right)^{\frac{1}{2}} \\
& \left.\leq R_{z}^{-1}\left(\sum_{\lambda_{j} \in \Lambda} \varphi_{j}(w)^{2} e^{-2 \lambda_{j} t}\right)^{\frac{1}{2}}\left(\sum_{\lambda_{j} \in \Lambda} f_{B\left(z, \frac{1}{2} \delta_{0} R_{z}\right)}\left|\varphi_{j}\right|^{2}\right)^{\frac{1}{2}}\right)^{\frac{1}{2}}\left|\varphi_{j}\right|^{2} \\
& \leq R_{z}^{-1} K_{2 t}(w, w)^{\frac{1}{2}}\left(\sum_{\lambda_{j} \in \Lambda} f_{B\left(z, \frac{1}{2} \delta_{0} R_{z}\right)}\right.
\end{aligned}
$$

giving, with constant depending on $C_{1}, C_{1}^{\prime}, C_{2}, C_{2}^{\prime}, C_{6},\left\{d, c_{\min }, c_{\max },\|g\|_{\alpha}, \alpha\right\}$,

$$
t^{\frac{-d}{2}}\left(\frac{R_{z}^{2}}{t}\right)^{2} \lesssim \sum_{\lambda_{j} \in \Lambda} f_{B_{\frac{1}{2} \delta_{0} R_{z}}(z)}\left|\varphi_{j}\right|^{2}
$$

Thus, by the pigeon-hole principle and Weyl's bound (2.1.2), we have $\lambda_{j} \in \Lambda_{1}$ with

$$
\frac{1}{C_{\text {count }}}\left(\frac{R_{z}^{2}}{t}\right)^{2} \lesssim f_{B_{\frac{1}{2} \delta_{0} R_{z}}(z)}\left|\varphi_{j}\right|^{2} .
$$

This gives equation (3.1.14).

3.5. Supplemental lemmata for the manifold case. We will initially be interested in localizing the manifold Laplacian $\Delta_{\mathscr{M}}$ to a ball $B=B_{R}(z), R \leq r_{U}(z)$, in a coordinate chart about $z$, satisfying the assumptions in the Theorem. We will rescale up so that $R=1$ (and rescale the volume of $\mathscr{M}$ accordingly). We impose Dirichlet boundary conditions on $\partial B$, and denote by $\tilde{\Delta}^{B}$ this Laplacian, which has the expression (2.2.3). We will compare $\tilde{\Delta}^{B}$ with the Euclidean Laplacian $\Delta^{B}$ on $B$ (also with Dirichlet boundary conditions). We will then compare $\tilde{\Delta}^{B}$ with the global Laplacian $\Delta_{\mathscr{M}}$ on the whole manifold (with Dirichlet or Neumann boundary conditions). The first comparison is most conveniently done through the associated Green functions. We use the following notation:

(i) $\Delta^{B} \xi_{j}=\mu_{j} \xi_{j}$ is the eigen-decomposition of $\Delta^{B}$, with sorted eigenvalues $0 \leq$ $\mu_{0} \leq \mu_{1} \leq \ldots, \tilde{\Delta}^{B} \tilde{\xi}_{j}=\tilde{\mu}_{j} \tilde{\xi}_{j}$ is the analogous decomposition of $\tilde{\Delta}^{B}$, and $\Delta_{\mathscr{M}} \varphi_{j}=\lambda_{j} \varphi_{j}$ the one for $\Delta_{\mathscr{M}}$. The eigenfunctions are assumed to be normalized in the corresponding natural $L^{2}$ spaces;

(ii) $G^{B}$ is the Green function on $B$, associated with $\Delta^{B}$, with Dirichlet boundary conditions, and $K^{B}$ the corresponding heat kernel; 
(iii) $\tilde{G}^{B}$ is the Green function on $B$, associated with $\tilde{\Delta}^{B}$, with Dirichlet boundary conditions, and $\tilde{K}^{B}$ the corresponding heat kernel;

(iv) the quadratic form associated with $g$, restricted to $B$, will be abbreviated as

$$
g_{B}(u, v)=\int_{B} \sum_{i, j=1}^{d} g^{i j} \partial_{i} u \partial_{j} v .
$$

for suitable $u, v$.

We will use estimates from [24], where they are stated only for the case of $d \geq 3$. Our Theorems are true also for the case $d=2$ (and trivially, $d=1$ ). This can be seen indirectly by considering $\tilde{\mathscr{M}}:=\mathscr{M} \times \mathbf{T}$ and noting that the eigenfunctions of $\tilde{\mathscr{M}}$ and the heat kernel of $\tilde{\mathscr{M}}$ both factor.

We let

$$
\left(L^{*}\right)^{p}(B)=\left\{f: B \rightarrow \mathbf{R} \text { measurable: }\|f\|_{\left(L^{*}\right)^{p}}<+\infty\right\}
$$

where

$$
\|f\|_{\left(L^{*}\right)^{p}}=\sup _{t>0} t|\{x \in B:|f(x)|>t\}|^{\frac{1}{p}} .
$$

We recall the following Theorem from [24].

Theorem 3.5.1. Suppose $d \geq 3$, and $g \in L^{\infty}$ and uniformly elliptic with $c_{\text {min }}$ and $c_{\max }$ as in (2.2.1). There exists a unique nonnegative function $\tilde{G}^{B}: B \rightarrow \mathbf{R} \cup\{\infty\}$, called the Green function, such that for each $y \in B$ and any $r>0$ such that $B_{r}(y) \subseteq B$,

$$
\tilde{G}^{B}(\cdot, y) \in W_{c}^{1,2}\left(B \backslash B_{r}(y)\right) \cap W_{c}^{1,1}(B),
$$

$\left.G^{B}\right|_{\partial B}=0$, and for all $\phi \in \mathscr{C}_{c}^{\infty}(B)$

$$
g_{B}\left(\tilde{G}^{B}(\cdot, y), \phi\right)=\phi(y) .
$$

Moreover, for each $y \in B$,

(i) $\tilde{G}^{B}(\cdot, y) \in\left(L^{*}\right)^{\frac{d}{d-2}}$, with , $\left\|\tilde{G}^{B}\right\|_{\left(L^{*}\right) \frac{d}{d-2}} \lesssim_{d, c_{\min }} 1$,

(ii) $\nabla \tilde{G}^{B}(\cdot, y) \in\left(L^{*}\right)^{\frac{d}{d-1}}$, with $\left\|\nabla \tilde{G}^{B}\right\|_{\left(L^{*}\right) \frac{d}{d-1}} \lesssim_{d, c_{\max }, c_{\min }} 1$,

(iii) $\tilde{G}^{B}(x, y){\gtrsim d, c_{\max }, c_{\min }}|x-y|^{2-d}$ for $|x-y| \leq \frac{1}{2} d(y, \partial B)$,

$$
\tilde{G}^{B}(x, y) \lesssim_{d, c_{\max }, c_{\min }}|x-y|^{2-d} .
$$

If $g \in \mathscr{C}^{\alpha}$, we also have (see page 333 in [24])

$$
\nabla_{y} \tilde{G}^{B}(x, y) \lesssim_{d, c_{\max }, c_{\min }, \alpha,\|g\|_{\alpha}}|x-y|^{1-d}
$$

(vi)

$$
\begin{aligned}
& \left|\nabla_{x} \tilde{G}^{B}\left(x_{1}, y\right)-\nabla_{x} \tilde{G}^{B}\left(x_{2}, y\right)\right| \\
& \lesssim_{d, c_{\max }, c_{\min }, R^{\alpha}, \alpha,\|g\|_{\alpha}} \frac{\left|x_{1}-x_{2}\right|^{\alpha}}{\left|x_{1}-y\right|^{d+\alpha-1}+\left|x_{2}-y\right|^{d+\alpha-1}} .
\end{aligned}
$$


Simple consequences of the bounds above are the following inequalities, which we record for future use:

$$
\begin{gathered}
\int_{c_{1} R \leq\|z-y\| \leq c_{2} R}\left|\tilde{G}^{R}(z, y)\right|^{p} d y \lesssim_{c_{1}, c_{2}, d, c_{\min }, c_{\max }, p} R^{(2-d)+\frac{d}{p}}, \\
\int_{c_{1} R \leq\|z-y\| \leq c_{2} R}\left|\nabla_{y} \tilde{G}^{R}(z, y)\right|^{p} d y \lesssim_{c_{1}, c_{2}, d, c_{\min }, c_{\max }, \alpha,\|g\|_{\alpha}, p} R^{(1-d)+\frac{d}{p}}, \\
\int_{B}\left|\nabla_{y} \tilde{G}^{R}(z, y)\right| d y \lesssim_{d, c_{\min }, c_{\max }, \alpha,\|g\|_{\alpha}} R,
\end{gathered}
$$

which are an immediate consequence of (3.5.3), and are valid for $c_{1}, c_{2}>0$ and $0<R_{z}^{\prime}<R_{z}$.

We recall that if we only assume uniform ellipticity, without any assumption on the modulus of continuity of $g$, then we have no pointwise estimates on $\nabla G$.

3.5.1. Perturbation of eigenfunctions. We start by comparing eigenfunctions of the Euclidean $\Delta^{B}$ with eigenfunctions of $\tilde{\Delta}^{B}$. We remind the reader that we have rescaled up to $R=1$.

Lemma 3.5.2. Let $J>0$ and $\eta>0$ be given. There is an $\epsilon_{0}=\epsilon_{0}(J)$ so that if $\epsilon<\epsilon_{0}$ and $\operatorname{Id}:\left(B, \delta^{i j}\right) \rightarrow\left(B, g^{i j}\right)$ is $1+\epsilon$ bi-Lipschitz, then for $j<J$,

$$
\left\|\xi_{j}-\tilde{\xi}_{j}\right\|_{L^{2}(B)} \leq \eta, \quad\left|\mu_{j}-\tilde{\mu}_{j}\right|<\eta \mu_{j}
$$

Proof. This follows from Lemma 20 in [23].

Lemma 3.5.3. There is an integer $\beta_{\mathrm{loc}}>0$ such that the following bounds hold:

$$
\left\|\tilde{\xi}_{j}\right\|_{L^{\infty}(B)} \lesssim d, c_{\min }, c_{\max }\left(\tilde{\mu}_{j}\right)^{\beta_{\mathrm{loc}}}
$$

and if $g^{i j} \in \mathscr{C}^{\alpha}$, we also have

$$
\left\|\nabla_{y} \tilde{\xi}_{j}\right\|_{L^{\infty}(B)} \lesssim d, c_{\min }, c_{\max }, \alpha,\|g\|_{\alpha} \tilde{\mu}_{j}\left(\tilde{\mu}_{j}\right)^{\beta_{\mathrm{loc}}}
$$

with $\beta_{\mathrm{loc}}=\frac{d-1}{2}$ for $d$ odd and $\beta_{\mathrm{loc}}=\frac{d}{2}$ for $d$ even.

Proof. By the definition of $\tilde{G}$, and by recalling that $\left.\tilde{G}\right|_{\partial B}=0$,

$$
\tilde{\xi}_{j}=\tilde{G}^{B} \tilde{\Delta}^{B} \tilde{\xi}_{j}=\tilde{\mu}_{j} \tilde{G}^{B} \tilde{\xi}_{j}=\ldots=\tilde{\mu}_{j}^{k} \underbrace{\tilde{G}^{B} \ldots \tilde{G}^{B}}_{k} \tilde{\xi}_{j} .
$$

Let $p_{i}, q_{i}$ be such that

$$
\sum_{1 \leq i \leq k} p_{i}^{-1}-k+1=q_{k}^{-1}
$$

Then, using Young's inequality we have

$$
\begin{aligned}
\|\underbrace{\tilde{G}^{B} * \cdots * \tilde{G}^{B}}_{k}\|_{L^{q_{k}}} & \lesssim_{q_{k-1}, q_{k}, p_{k}}\|\underbrace{\tilde{G}^{B} * \cdots * \tilde{G}^{B}}_{k-1}\|_{L^{q_{k-1}}}\left\|\tilde{G}^{B}\right\|_{L^{p_{k}}} \\
& \lesssim q_{q_{k-2}, q_{k-1}, p_{k-1}} \ldots \lesssim_{q_{1}, q_{2}, p_{2}}\left\|\tilde{G}^{B}\right\|_{L^{p_{1}}} \ldots\left\|\tilde{G}^{B}\right\|_{L^{p_{k}}} .
\end{aligned}
$$

We have $\tilde{G}^{B} \in L^{\frac{d-1}{d-2}}$ by Theorem 3.5.1; we take $p_{i}=\frac{d-1}{d-2}$ and take $k=d-1$ and get

$$
q_{d-1}^{-1}=(d-1) \frac{d-2}{d-1}-(d-1)+1=0,
$$


for odd $d$

and for even $d$

$$
q_{\frac{d-1}{2}}^{-1}=\frac{d-1}{2} \frac{d-2}{d-1}-\frac{d-1}{2}+1=\frac{1}{2}
$$

$$
q_{\frac{d}{2}}^{-1}=\frac{d}{2} \frac{d-2}{d-1}-\frac{d}{2}+1=\frac{d}{2}\left(\frac{d-2}{d-1}-1\right)+1 \leq \frac{1}{2} .
$$

Now, for odd $d$,

$$
\left\|\tilde{\xi}_{j}\right\|_{\infty} \lesssim \tilde{\mu}_{j}^{\frac{d-1}{2}}\|\underbrace{\tilde{G}^{B} * \ldots * \tilde{G}^{B}}_{\frac{d-1}{2}}\|_{L^{2}}\left\|\tilde{\xi}_{j}\right\|_{L^{2}} \leq \tilde{\mu}_{j}^{\frac{d-1}{2}}\left\|\tilde{G}^{B}\right\|_{L^{\frac{d-1}{d-2}}} \lesssim \tilde{\mu}_{j}^{\frac{d-1}{2}}
$$

which gives the first desired bound. If $d$ is even, do the same with $\frac{d}{2}$ replacing $\frac{d-1}{2}$.

For the gradient estimate, we have

$$
\left|\nabla_{y} \tilde{\xi}_{j}\right|=\left|\nabla_{y} g_{B}\left(\tilde{G}^{B}, \tilde{\xi}_{j}\right)\right|=\left|\nabla_{y} \int_{B} \tilde{G}^{B} \tilde{\Delta}^{B} \tilde{\xi}_{j}\right|=\left|\tilde{\mu}_{j} \nabla_{y} \int \tilde{G}^{B} \tilde{\xi}_{j}\right| \leq \tilde{\mu}_{j}\left\|\nabla_{y} \tilde{G}^{B}\right\|_{L^{1}}\left\|\tilde{\xi}_{j}\right\|_{\infty}
$$

where we used the defining property of $\tilde{G}^{B}$ in Theorem 3.5.1 and Green's Theorem. We estimate the last term by (3.5.8) and equation (3.5.3) to get the desired result.

We can now convert the $L^{2}$-estimates in Lemma 3.5.2 into $L^{\infty}$-estimates. We will need the following

Lemma 3.5.4. Assume that $\left|g^{i j}(x)-\delta^{i j}\right|<\epsilon$ for $x \in B$. Then for $\psi \in C_{c}^{\infty}(B)$ we have

$$
\left\|\int_{B}\left\langle\nabla\left(\tilde{G}^{B}(z, w)-G^{B}(z, w)\right), \nabla \psi(z)\right\rangle d z\right\|_{L^{\infty}(B)} \leq \epsilon\left\|\nabla_{y} \tilde{G}^{B}\right\|_{L^{1}}\|\nabla \psi\|_{L^{\infty}(B)},
$$

and if $g^{i j} \in \mathscr{C}^{\alpha}$, we also have

$$
\left\|\int_{B}\left\langle\nabla\left(\tilde{G}^{B}(z, w)-G^{B}(z, w)\right), \nabla \tilde{\xi}_{l}(z)\right\rangle d z\right\|_{L^{\infty}(B)} \lesssim_{d, c_{\min }, c_{\max }, \alpha,\|g\|_{\alpha}}^{\epsilon \tilde{\mu}_{l}\left(\tilde{\mu}_{l}\right)^{\beta_{\mathrm{loc}}}}
$$

as well as

$$
\left\|\int_{B}\left\langle\nabla\left(\tilde{G}^{B}(z, w)-G^{B}(z, w)\right), \nabla \xi_{l}(z)\right\rangle d z\right\|_{L^{\infty}(B)} \lesssim_{d, c_{\min }, c_{\max }, \alpha,\|g\|_{\alpha}} \epsilon \mu_{l}\left(\mu_{l}\right)^{\beta_{\mathrm{loc}}}
$$

with $\beta_{\text {loc }}$ as in Lemma 3.5.3.

Proof. Now

$$
\begin{aligned}
& \left\|\int_{B}\left\langle\nabla\left(G^{B}(z, w)-\tilde{G}^{B}(z, w)\right), \nabla \psi(z)\right\rangle d z\right\|_{L^{\infty}(B)} \\
& =\left\|\int_{B} \sum_{i, j} \delta^{i j} \partial_{i}\left(G^{B}(z, w)-\tilde{G}^{B}(z, w)\right) \partial_{j} \psi(z) d z\right\|_{L^{\infty}(B)} \\
& =\| \int_{B} \sum_{i, j}\left(\delta^{i j} \partial_{i} G^{B}(z, w)-g^{i j}(z) \partial_{i} \tilde{G}^{B}(z, w)\right) \partial_{j} \psi(z) d z \\
& \quad+\int_{B} \sum_{i, j}\left(g^{i j}(z) \partial_{i} \tilde{G}^{B}(z, w)-\delta^{i j} \partial_{i} \tilde{G}^{B}(z, w)\right) \partial_{j} \psi(z) d z \|_{L^{\infty}(B)} \\
& =\left\|\int_{B} \sum_{i, j}\left(\left(g^{i j}(z)-\delta^{i j}\right) \partial_{i} \tilde{G}^{B}(z, w)\right) \partial_{j} \psi(z) d z\right\|_{L^{\infty}(B)}
\end{aligned}
$$




$$
\lesssim \epsilon\left\|\nabla_{y} \tilde{G}^{B}\right\|_{L^{1}}\|\nabla \psi\|_{L^{\infty}(B)}
$$

which gives (3.5.10). Using Lemma 3.5.3 one also gets (3.5.11) and (3.5.12).

Lemma 3.5.5. Let $J, \eta>0$ be given. Let $\beta_{\mathrm{loc}}$ be as in Lemma 3.5.3. There is an $\epsilon_{0}$ which depends on $\left.J, \eta, d, c_{\max }, c_{\min },\|g\|_{\alpha}, \alpha\right)$, so that if $\epsilon<\epsilon_{0}$, and $\left|g^{i l}(x)-\delta^{i l}\right|<\epsilon$ for $x \in B$, then for $j<J$,

$$
\begin{gathered}
\left|\mu_{j}-\tilde{\mu}_{j}\right|<\eta \mu_{j} \\
\left\|\xi_{j}-\tilde{\xi}_{j}\right\|_{L^{\infty}(B)} \lesssim_{d, c_{\min }, c_{\max },\|g\|_{\alpha}, \alpha} \eta Q_{1}\left(\tilde{\mu}_{l}\right),
\end{gathered}
$$

where $Q_{1}$ is a polynomial of degree $2 \beta_{\text {loc }}$. If $g \in \mathscr{C}^{\alpha}$, we also have

$$
\left\|\nabla\left(\xi_{j}-\tilde{\xi}_{j}\right)\right\|_{L^{\infty}} \lesssim_{d, c_{\min }, c_{\max },\|g\|_{\alpha}, \alpha} \eta Q_{2}\left(\tilde{\mu}_{l}\right)
$$

where $Q_{2}$ is a polynomial of degree $2 \beta_{\text {loc }}+1$.

Proof. The bound (3.5.13) follows from Lemma 3.5.2. Let $q_{i}$ and $p_{i}$ be as in the proof of Lemma 3.5.3.

We have using the definitions of $G^{B}$ and $\tilde{G}^{B}$

$$
\begin{aligned}
\xi_{l}(w)-\tilde{\xi}_{l}(w)= & \int_{B} \sum_{i, j} \delta^{i j} \partial_{i} G^{B}(z, w) \partial_{j} \xi_{l}(z)-g^{i j}(z) \partial_{i} \tilde{G}^{B}(z, w) \partial_{j} \tilde{\xi}_{l}(z) d z \\
= & \int_{B} \sum_{i, j} \delta^{i j}\left(\partial_{i} G^{B}(z, w) \partial_{j} \xi_{l}(z)-\partial_{i} \tilde{G}^{B}(z, w) \partial_{j} \xi_{l}(z)\right) \\
& +\left(\delta^{i j} \partial_{i} \tilde{G}^{B}(z, w) \partial_{j} \tilde{\xi}_{l}(z)-g^{i j}(z) \partial_{i} \tilde{G}^{B}(z, w) \partial_{j} \xi_{l}(z)\right) d z \\
= & E^{1}(w)+\int_{B} \sum_{i, j}\left(\partial_{i} \tilde{G}^{B}(z, w) \partial_{j} \xi_{l}(z)-g^{i j}(z) \partial_{i} \tilde{G}^{B}(z, w) \partial_{j} \tilde{\xi}_{l}(z)\right) d z \\
= & E^{1}(w)+\mu_{l} \cdot \tilde{G}^{B} * \xi_{l}(w)-\tilde{\mu}_{l} \cdot \tilde{G}^{B} * \tilde{\xi}_{l}(w) \\
= & E^{1}(w)+\left(\mu_{l}-\tilde{\mu}_{l}\right) \cdot \tilde{G}^{B} * \xi_{l}(w)+\tilde{\mu}_{l} \cdot \tilde{G}^{B} *\left(\xi_{l}-\tilde{\xi}_{l}\right)(w) \\
= & E^{1}(w)+E^{2}(w)+\tilde{\mu}_{l} \cdot \tilde{G}^{B} *\left(\xi_{l}-\tilde{\xi}_{l}\right)(w),
\end{aligned}
$$

where we have from equation (3.5.12)

$$
\left\|E^{1}\right\|_{L^{\infty}(B)} \lesssim_{d, c_{\min }, c_{\max }, \alpha,\|g\|_{\alpha}} \epsilon \mu_{l}\left(\mu_{l}\right)^{\beta_{\mathrm{loc}}}
$$

and

$$
\left\|E^{2}\right\|_{L^{\infty}(B)} \lesssim_{d, c_{\min }, c_{\max }, \alpha,\|g\|_{\alpha}} \eta \mu_{l} \cdot\left(\mu_{l}\right)^{\beta_{\mathrm{loc}}} .
$$

Iterating, we have

$$
\begin{aligned}
& \left|\xi_{l}(w)-\tilde{\xi}_{l}(w)\right|=\left|E^{1}(w)+E^{2}(w)+\tilde{\mu}_{l} \cdot \tilde{G}^{B} *\left(\xi_{l}-\tilde{\xi}_{l}\right)(w)\right| \\
& =\left|E^{1}(w)+E^{2}(w)+\tilde{\mu}_{l} \cdot \tilde{G}^{B} *\left(E^{1}+E^{2}+\tilde{\mu}_{l} \cdot \tilde{G}^{B} *\left(\xi_{l}-\tilde{\xi}_{l}\right)\right)(w)\right| . \\
& =\ldots \\
& \leq\left\|E^{1}+E^{2}\right\|_{L^{\infty}(B)} \sum_{k=0}^{\beta_{\mathrm{loc}}-1}\left(\tilde{\mu}_{l}\left\|\tilde{G}^{B}\right\|_{L^{1}(B)}\right)^{k}+\tilde{\mu}_{l}^{\beta_{\mathrm{loc}}}|\underbrace{\tilde{G}^{B} * \ldots \tilde{G}^{B}}_{\beta_{\text {loc }} \text { times }} *\left(\xi_{l}(w)-\tilde{\xi}_{l}(w)\right)|
\end{aligned}
$$




$$
\begin{aligned}
& \leq\left\|E^{1}+E^{2}\right\|_{L^{\infty}(B)} \sum_{k=0}^{\beta_{\mathrm{loc}}-1}\left(\tilde{\mu}_{l}\left\|\tilde{G}^{B}\right\|_{L^{1}(B)}\right)^{k}+\tilde{\mu}_{l}^{\beta_{\mathrm{loc}}}\left\|\tilde{G}^{B}\right\|_{L^{\frac{d-1}{d-2}}}\left\|\xi_{l}-\tilde{\xi}_{l}\right\|_{2} \\
& \lesssim 2 \eta \mu_{l}\left(\mu_{l}\right)^{\beta_{\mathrm{loc}}} \sum_{k=0}^{\beta_{\mathrm{loc}}-1} \tilde{\mu}_{l}^{k}+\eta \cdot \tilde{\mu}_{l}^{\beta_{\mathrm{loc}}}=\eta Q_{1}\left(\tilde{\mu}_{l}\right),
\end{aligned}
$$

where we require for the penultimate inequality $\epsilon<\eta$.

To prove the gradient estimate,

$$
\begin{aligned}
\left.\mid \nabla\left(\tilde{\xi}_{j}-\xi_{j}\right)\right)(y) \mid= & \left|\nabla_{y} \int \sum_{i, l} \partial_{z_{i}} \tilde{G}^{R}(z, y) g^{i l} \partial_{z_{l}} \tilde{\xi}_{j}(z)-\sum_{i, l} \partial_{z_{i}} G^{B}(z, y) \delta^{i l} \partial_{z_{l}} \xi_{j}(z)\right| \\
= & \left|\nabla_{y} \int \tilde{G}^{R}(z, y) \tilde{\mu}_{j} \tilde{\xi}_{j}(z)-G^{B}(z, y) \mu_{j} \xi_{j}(z)\right| \\
= & \left|\int \nabla_{y} \tilde{G}^{R}(z, y) \tilde{\mu}_{j} \tilde{\xi}_{j}(z)-\nabla_{y} G^{B}(z, y) \mu_{j} \xi_{j}(z)\right| \\
\leq & \int\left|\nabla_{y}\left(\tilde{G}^{R}-G^{B}\right)(z, y)\right| \cdot\left|\tilde{\mu}_{j} \tilde{\xi}_{j}(z)\right| \\
& +\left|\nabla_{y} G^{B}(z, y)\right| \cdot\left|\tilde{\mu}_{j} \tilde{\xi}_{j}(z)-\mu_{j} \xi_{j}(z)\right| .
\end{aligned}
$$

Now using equation (3.5.14), Lemma 3.5.2, and Theorem 3.5.1 we get equation (3.5.15).

3.5.2. Bounds on eigenfunctions. The main goal of this section is to prove Proposition 3.4.1. We note that the inequalities (3.4.1), (3.4.2), and (3.4.3) are invariant under scalings of the metric, and so, once again, we assume in the proof of this Proposition and in all the Lemmata that $R=1$. In this section all constants subsumed in $\lesssim$ and $\gtrsim$ will in general depend on $d, c_{\min }, c_{\max },\|g\|_{\alpha}, \alpha$. We will need the following result.

Lemma 3.5.6. (Lemma 3.1 from [24]) Suppose $h$ is a bounded solution of $\tilde{\Delta}^{B} h=$ 0 in $B$. Then

$$
|\nabla h(x)| \lesssim_{d, c_{\min }, c_{\max },\|g\|_{\alpha}, \alpha}(1-\operatorname{dist}(x, z))^{-1}\|h\|_{L^{\infty}(B)}
$$

Lemma 3.5.7. Assume that $g \in \mathscr{C}^{\alpha}$ and $\tilde{\Delta}^{B} h=0$ on $B=B_{1}(z)$. Then for any $r<1$

and

$$
\|h\|_{L^{\infty}\left(B_{r}(z)\right)} d_{d, c_{\min }, c_{\max }} C_{r}\|h\|_{L^{2}\left(B_{2 r}(z)\right)}
$$

$$
\|\nabla h\|_{L^{\infty}\left(B_{\frac{r}{2}}(z)\right)} \lesssim_{d, c_{\min }, c_{\max },\|g\|_{\alpha}, \alpha} C_{r}^{\prime}\|h\|_{L^{2}\left(B_{2 r}(z)\right)} .
$$

Proof. Let $r$ as above be given. Fix $0<a_{1}<a_{2}<1$. By the coarea formula [22], we have

$$
\begin{aligned}
& \int_{a_{1}}^{a_{2}} \int_{\left\{x \in B_{r}(z): \tilde{G}^{r}(x)=t\right\}}|h(x)| d \mathscr{H}^{d-1}(x) d t=\int_{\left\{x \in B_{r}(z): a_{1}<\tilde{G}^{r}(x)<a_{2}\right\}}\left|h \nabla \tilde{G}^{r}\right| d x \\
& \leq\|h\|_{L^{2}\left(B_{r}(z)\right)}\left\|\left|\nabla \tilde{G}^{r}\right|\right\|_{L^{2}\left(B_{C\left(d, \frac{c \max }{c_{\min }} a_{2}^{d-2}\right)}(z) \backslash B_{c\left(d, \frac{c_{\max }}{c_{\min }} a_{1}^{d-2}\right)}(z)\right)} \lesssim\|h\|_{L^{2}\left(B_{r}(z)\right)}
\end{aligned}
$$


by estimate (3.5.6). Hence there exists $t^{*} \in\left[a_{1}, a_{2}\right]$ such that

$$
\int_{\left\{x \in B_{r}(z): \tilde{G}^{r}=t^{*}\right\}}|h(x)| d \mathscr{H}^{d-1}(x) \lesssim\|h\|_{L^{2}\left(B_{r}(z)\right)}\left(a_{2}-a_{1}\right)^{-1} .
$$

Now, by $\tilde{\Delta}^{B}$ harmonicity,

$$
\begin{aligned}
|h(z)| & =\left|\int_{\left\{y \in B_{r}(z): \tilde{G}^{r}(y)=t^{*}\right\}} h(y) \frac{\partial \tilde{G}^{r}}{\partial n}(z, y) d \mathscr{H}^{d-1}(y)\right| \\
& \leq\left\|\nabla \tilde{G}^{r}\right\|_{L^{\infty}\left(\left\{\tilde{G}^{r}(y)=t^{*}\right\}\right)} \int_{\left\{y \in B_{r}(z): \tilde{G}^{r}=t^{*}\right\}}|h(y)| d \mathscr{H}^{d-1}(y) \\
& \lesssim\|h\|_{L^{2}\left(B_{r}(z)\right)} \lesssim_{a_{1}, a_{2}, d, c_{\min }, c_{\max }}\|h\|_{L^{2}\left(B_{r}(z)\right)} .
\end{aligned}
$$

Essentially the same proof holds if we replace $z$ by $\left.w \in B_{r}(z)\right)$ in the above estimates, giving the desired bound on $h$.

In order to estimate the gradient, we use Lemma 3.5.6, which gives us

$$
\|\nabla h\|_{L^{\infty}\left(B_{\frac{r}{2}}(z)\right)} d_{d, c_{\min }, c_{\max },\|g\|_{\alpha}, \alpha} C_{r}^{\prime}\|h\|_{L^{\infty}\left(B_{r}(z)\right)},
$$

which implies the desired estimate.

Lemma 3.5.8. Assume that $g \in \mathscr{C}^{\alpha}$. Let $\tilde{\xi}_{j}$ and $\varphi_{k}$ be as above. Then we have the estimate

$$
\left\|\tilde{\xi}_{j} \varphi_{k}\right\|_{L^{\frac{2 d}{d-2}}(B)} \lesssim_{d, c_{\min }, c_{\max },\|g\|_{\alpha}, \alpha}\left(\left(\left(\tilde{\mu}_{j}+\lambda_{k}\right)\right)^{\frac{1}{2}}+\tilde{\mu}_{j}\right)\left(\tilde{\mu}_{j}\right)^{\beta_{\mathrm{loc}}}\left\|\varphi_{k}\right\|_{L^{2}(B)} .
$$

Proof. By the Sobolev embedding Theorem it is enough to prove that

$$
\left\|\nabla\left(\tilde{\xi}_{j} \varphi_{k}\right)\right\|_{L^{2}(B)} \lesssim\left(\left(\left(\tilde{\mu}_{j}+\lambda_{k}\right)\right)^{\frac{1}{2}}+\tilde{\mu}_{j}\right)\left(\tilde{\mu}_{j}\right)^{\beta_{\mathrm{loc}}}\left\|\varphi_{k}\right\|_{L^{2}(B)} .
$$

To this end, first note that we may write

$$
\begin{aligned}
\tilde{\Delta}^{B}\left(\tilde{\xi}_{j} \varphi_{k}\right) & =\varphi_{k} \tilde{\Delta}^{B} \tilde{\xi}_{j}+\tilde{\xi}_{j} \tilde{\Delta}^{B} \varphi_{k}+\sum_{i, j=1}^{d} g^{i j} \partial_{i} \varphi_{k} \partial_{j} \tilde{\xi}_{j} \\
& =\left(\tilde{\mu}_{j}+\lambda_{k}\right) \varphi_{k} \tilde{\xi}_{j}+\sum_{i, j=1}^{d} g^{i j} \partial_{i} \varphi_{k} \partial_{j} \tilde{\xi}_{j}
\end{aligned}
$$

and so $\tilde{\Delta}^{B}\left(\tilde{\xi}_{j} \varphi_{k}\right)$ is defined as a function, and not just a distribution. Observe that $\varphi_{l}$ does not satisfy any particular boundary condition on $\partial B$, however since $\tilde{\xi}_{j}=0$ on $\partial B$, integration by parts gives

$$
\left\langle\tilde{\xi}_{j} \varphi_{k}, \tilde{\Delta}^{B}\left(\tilde{\xi}_{j} \varphi_{k}\right)\right\rangle_{B}=g_{B}\left(\nabla\left(\tilde{\xi}_{j} \varphi_{k}\right), \nabla\left(\tilde{\xi}_{j} \varphi_{k}\right)\right) .
$$

Now, since $g$ is a positive quadratic form,

$$
\begin{aligned}
\left\langle\nabla\left(\tilde{\xi}_{j} \varphi_{k}\right), \nabla\left(\tilde{\xi}_{j} \varphi_{k}\right)\right\rangle_{g_{B}} & =\left\langle\tilde{\xi}_{j} \nabla \varphi_{k}+\varphi_{k} \nabla \tilde{\xi}_{j}, \tilde{\xi}_{j} \nabla \varphi_{k}+\varphi_{k} \nabla \tilde{\xi}_{j}\right\rangle_{g_{B}} \\
& \geq\left\langle\tilde{\xi}_{j} \nabla \varphi_{k}, \tilde{\xi}_{j} \nabla \varphi_{k}\right\rangle_{g_{B}}-2\left|\left\langle\varphi_{k} \nabla \tilde{\xi}_{j}, \tilde{\xi}_{j} \nabla \varphi_{k}\right\rangle_{g_{B}}\right|
\end{aligned}
$$


and therefore

$$
\begin{aligned}
& g_{B}\left(\tilde{\xi}_{j} \nabla \varphi_{k}, \tilde{\xi}_{j} \nabla \varphi_{k}\right) \leq g_{B}\left(\nabla\left(\tilde{\xi}_{j} \varphi_{k}\right), \nabla\left(\tilde{\xi}_{j} \varphi_{k}\right)\right)+2\left|g_{R}\left(\varphi_{k} \nabla \tilde{\xi}_{j}, \tilde{\xi}_{j} \nabla \varphi_{k}\right)\right| \\
& =\left\langle\tilde{\xi}_{j} \varphi_{k}, \tilde{\Delta}^{B}\left(\tilde{\xi}_{j} \varphi_{k}\right)\right\rangle_{B}+2\left|g_{R}\left(\varphi_{k} \nabla \tilde{\xi}_{j}, \tilde{\xi}_{j} \nabla \varphi_{k}\right)\right| \\
& \leq\left\langle\tilde{\xi}_{j} \varphi_{k},\left(\left(\tilde{\mu}_{j}+\lambda_{k}\right) \tilde{\xi}_{j} \varphi_{k}\right)\right\rangle_{B}+4\left|g_{R}\left(\varphi_{k} \nabla \tilde{\xi}_{j}, \tilde{\xi}_{j} \nabla \varphi_{k}\right)\right| \\
& \lesssim\left(\tilde{\mu}_{j}+\lambda_{k}\right)\left\|\tilde{\xi}_{j}^{2}\right\|_{\infty}\left\|\varphi_{k}\right\|_{L^{2}(B)}^{2}+\left\|\nabla \tilde{\xi}_{j}\right\|_{\infty}\left\|\varphi_{k}\right\|_{L^{2}(B)} g_{B}\left(\tilde{\xi}_{j} \nabla \varphi_{k}, \tilde{\xi}_{j} \nabla \varphi_{k}\right)^{\frac{1}{2}} \\
& \lesssim\left(\tilde{\mu}_{j}+\lambda_{k}\right)\left(\tilde{\mu}_{j}\right)^{2 \beta_{\mathrm{loc}}}\left\|\varphi_{k}\right\|_{L^{2}(B)}^{2}+\tilde{\mu}_{j}\left(\tilde{\mu}_{j}\right)^{\beta_{\mathrm{loc}}}\left\|\varphi_{k}\right\|_{L^{2}(B)} g_{B}\left(\tilde{\xi}_{j} \nabla \varphi_{k}, \tilde{\xi}_{j} \nabla \varphi_{k}\right)^{\frac{1}{2}}
\end{aligned}
$$

giving

$$
g_{B}\left(\tilde{\xi}_{j} \nabla \varphi_{k}, \tilde{\xi}_{j} \nabla \varphi_{k}\right)^{\frac{1}{2}}=\left\|\tilde{\xi}_{j} \nabla \varphi_{k}\right\|_{L^{2}\left(B_{r}(z)\right)} \lesssim\left(\left(\tilde{\mu}_{j}+\lambda_{k}\right)^{\frac{1}{2}}+\tilde{\mu}_{j}\right)\left(\tilde{\mu}_{j}\right)^{\beta_{\mathrm{loc}}}\left\|\varphi_{k}\right\|_{L^{2}(B)} .
$$

Finally,

$$
\nabla\left(\tilde{\xi}_{j} \varphi_{k}\right) \leq\left|\varphi_{k} \| \nabla \tilde{\xi}_{j}\right|+\left|\tilde{\xi}_{j} \nabla \varphi_{k}\right|
$$

gives equation (3.5.20).

Proof of Proposition 3.4.1. We recall that we rescaled so that $R=1$. Let $\psi=\sum_{1}^{N} a_{j} \xi_{j}$ be a (finite) sum of (Euclidean) Dirichlet eigenfunctions of $B$ such that

$$
\frac{1}{2} \leq \psi(x) \leq 2 \quad \text { and } \quad x \in B_{R / 2}(z) \subsetneq B
$$

and $\sum_{1}^{N}\left|a_{j}\right| \leq C, \mu_{j} \leq C, 1 \leq j \leq N$. One may obtain such a sequence by taking $\psi^{\prime} \in C^{\infty}(B)$ with $0 \leq \psi^{\prime} \leq 1,\left.\psi^{\prime}\right|_{B_{R / 2}(z)}=1$ and $\left.\psi^{\prime}\right|_{\partial B(z)}=0$ and then take $\psi$ to be a truncation of the eigenfunction expansion of $\psi^{\prime}$. Let $\tilde{\psi}=\sum_{1}^{N} a_{j} \tilde{\xi}_{j}$ be the sum of the corresponding Dirichlet eigenfunctions for $B$ with respect to $\tilde{\Delta}^{B}$. By Lemma 3.5.5 and $\left|g^{i k}(x)-\delta^{i k}\right|<\epsilon$ (with $\epsilon$ sufficiently small), we have, for $x \in B_{R / 2}(z)$,

$$
\frac{1}{4} \leq \tilde{\psi}(x) \leq 3
$$

By Lemma 3.5.8

$$
\begin{aligned}
\left\|\varphi_{j}\right\|_{\left.L^{\frac{2 d}{d-2}}(B)\right)} & \leq\left\|\tilde{\psi} \varphi_{j}\right\|_{\left.L^{\frac{2 d}{d-2}}(B)\right)} \leq \sum\left|a_{i}\right|\left\|\tilde{\xi}_{i} \varphi_{j}\right\|_{L^{\frac{2 d}{d-2}}(B)} \\
& \lesssim \sum\left|a_{i}\right|\left(\left(\tilde{\mu}_{i}+\lambda_{j}\right)^{\frac{1}{2}}+\tilde{\mu}_{i}\right)\left(\tilde{\mu}_{i}\right)^{\beta_{\mathrm{loc}}\left\|\varphi_{j}\right\|_{L^{2}(B)}} \\
& \lesssim C\left(\lambda_{j}+1\right)^{\frac{1}{2}}\left\|\varphi_{j}\right\|_{L^{2}(B)} .
\end{aligned}
$$

We are now ready to prove inequality (3.4.1). Let $r_{0}=R=1>r_{1}>r_{2}>\cdots \geq$ $\frac{R}{2}=\frac{1}{2}$. Write $\left.\varphi_{j}\right|_{B_{r_{0}}(z)}$ on as $\left.\varphi_{j}\right|_{B_{r_{0}}(z)}=u+v$, where

$$
v=\tilde{G}^{B}\left(\tilde{\Delta}^{B} \varphi_{j}\right)=\lambda_{j} \tilde{G}^{B}\left(\varphi_{j}\right)
$$

since $\tilde{G}^{B}$ is the Green function for the Dirichlet problem on $B_{r_{0}}(z)$. Hence $\tilde{\Delta}^{B} u=0$. We use (see below) Lemma 3.5.7 in conjunction with the above decomposition, to show that $\varphi_{j} \in L^{\infty}\left(B_{r_{\infty}}(z)\right)$. We will then (see below) get (3.4.2) from differentiating (3.5.24) and using Lemma 3.5.7. Initially, by (3.5.23), Theorem 3.5.1, (3.5.24) and Young's inequality, with $p_{0}=\frac{2 d}{d-2}$ and $1 \leq p_{1}=\frac{2 d}{d-6+\eta_{1}}$ (with $0<\eta_{1}<4$ of our choice, implied by the estimates on the Green function in Theorem 3.5.1), we have

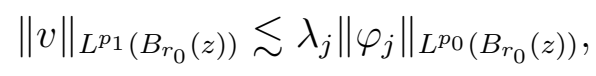


giving, by Lemma 3.5.7 ( since $p_{1}>p_{0}>2$ ),

$$
\|u\|_{L^{\infty}\left(B_{r_{1}}(z)\right)} \lesssim\|u\|_{L^{2}\left(B_{r_{0}}(z)\right)} \lesssim\left(1+\lambda_{j}\right)\left\|\varphi_{j}\right\|_{L^{2}\left(B_{r_{0}}(z)\right)} .
$$

Thus, we have

$$
\left\|\varphi_{j}\right\|_{L^{p_{1}}\left(B_{r_{1}}(z)\right)} \lesssim\left(1+\lambda_{j}\right)\left\|\varphi_{j}\right\|_{L^{p_{0}}\left(B_{r_{0}}(z)\right)} \lesssim\left(\lambda_{j}+1\right)^{\frac{3}{2}}\left\|\varphi_{j}\right\|_{L^{2}(B)} .
$$

Let $1 \leq p_{i}=\frac{2 d}{d-2-4 i+\sum_{k \leq i} \eta_{k}}$ (with $0<\eta_{i}<4$ of our choice) and $v_{i}=\tilde{G}^{r_{i}}\left(\tilde{\Delta}^{r_{i}} \varphi_{j}\right)$. Similarly, we have

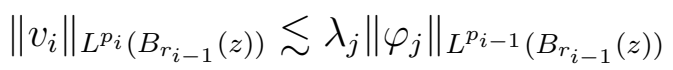

and for $u_{i}=\varphi_{j}-v_{i}$

$$
\left\|u_{i}\right\|_{L^{\infty}\left(B_{r_{i}}(z)\right)} \lesssim\|u\|_{L^{2}\left(B_{r_{i-1}}(z)\right)} \lesssim\left(1+\lambda_{j}\right)\left\|\varphi_{j}\right\|_{L^{2}\left(B_{r_{i-1}}(z)\right)} .
$$

Thus, we have by induction

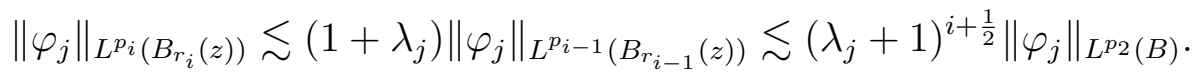

Let $\beta$ be the smallest integer larger or equal than $\frac{d-2}{4}$. We may choose $\left\{\eta_{i}\right\}$ so that $p_{\beta}=\infty$. This gives equation (3.4.1).

In order to upper bound $\left\|\nabla \varphi_{j}\right\|$ we note that (recalling that $r_{\beta} \sim R \sim 1$ )

$$
\left\|\nabla v_{\beta}\right\|_{L^{\infty}\left(B_{r_{\beta}}\right)}=\left\|\nabla \tilde{G}^{r_{\beta}}\left(\tilde{\Delta}^{r_{\beta}} \varphi_{j}\right)\right\|_{L^{\infty}\left(B_{r_{\beta}}\right)} \leq \lambda_{j}\left\|\tilde{G}^{r_{\beta}}\right\|_{L^{1}\left(B_{r_{\beta}}\right)}\|\varphi\|_{L^{\infty}\left(B_{r_{\beta}}\right)} .
$$

We also note that we have

$$
\left\|\nabla u_{\beta}\right\|_{L^{\infty}\left(B_{\frac{1}{2} r_{\beta}}\right)} \lesssim\|\varphi\|_{L^{\infty}\left(B_{r_{\beta}}\right)}
$$

from Lemma 3.5.7. Thus combining the last two estimates, we have (3.4.2).

Finally, we prove (3.4.3). Let $\chi \in C^{\infty}(\mathbf{R})$ be a function so that $0 \leq \chi \leq 1$, $\left.\chi(s)\right|_{s \leq K_{1}}=0$ and $\left.\chi(s)\right|_{s \geq K_{2}}=1$. We define $\eta$, a cutoff function, such that $\left.\eta\right|_{B\left(z, \frac{1}{4} R\right)}=$ 1 and $\left.\eta\right|_{|x| \geq \frac{1}{2} R}=0$ as follows. Define $\eta(x)=\chi(G(z, x))$, and choose $K_{1}, K_{2}$ above so that $\eta$ has the desired cutoff radius. We get that

$$
\begin{aligned}
\tilde{\Delta}^{B}(\eta)(x) & =\tilde{\Delta}(\chi(G(z, x)))=\sum_{i, j} \partial_{x_{i}} g^{i j} \partial_{x_{j}} \chi(G(z, x)) \\
& =\sum_{i, j} \partial_{x_{i}}\left(\chi^{\prime}(G(z, x))\right) g^{i j} \partial_{x_{j}} G(z, x) \\
& =\chi^{\prime \prime}(G(z, x))\left(\sum_{i, j} \partial_{x_{i}} G(z, x) g^{i j} \partial_{x_{j}} G(z, x)\right)+\chi^{\prime}(G(z, x)) \tilde{\Delta}_{x} G(z, x),
\end{aligned}
$$

where the second term in the last line is 0 as $\tilde{\Delta}_{x} G(z, x)$ is a distribution which equals 0 on the support of $\chi^{\prime}(G(z, x))$. By choice of $\chi$ and Theorem 3.5.1, this gives $\tilde{\Delta}(\eta) \lesssim 1$.

Now, let $x, y \in B=B\left(z, \frac{1}{4} R\right)$. Let $\tilde{G}=\tilde{G}^{B}$ be the Green's function for $B$. Then

$$
\begin{aligned}
\|\nabla \varphi(x)-\nabla \varphi(y)\| & =\| \nabla(\varphi \eta)(x)-\nabla \varphi \eta)(y) \| \\
& =\left\|\int\left(\nabla_{1} G(x, w)-\nabla_{1} G(y, w)\right) \tilde{\Delta}^{B}(\varphi \eta)(w) d w\right\| \\
& \leq\left|\tilde{\Delta}^{B}(\varphi \eta)(w)\right|_{L^{\infty}(B)} \int\left\|\nabla_{1} G(x, w)-\nabla_{1} G(y, w)\right\| d w .
\end{aligned}
$$


We have (using uniform ellipticity as well as Proposition 3.4.1)

$$
\begin{aligned}
\left|\tilde{\Delta}^{B}(\varphi \eta)(w)\right| & \lesssim\left|\eta \tilde{\Delta}^{B} \varphi(w)\right|+\left|\varphi(w) \tilde{\Delta}^{B} \eta\right|+|\nabla \eta \| \nabla \varphi| \\
& \leq\left(\left\|\tilde{\Delta}^{B} \eta\right\|_{\infty}+\lambda\|\eta\|_{\infty}\right)\|\varphi\|_{L^{\infty}(B)}+\|\nabla \eta\|_{\infty}\|\nabla \varphi\|_{L^{\infty}(B)} \\
& \lesssim(1+\lambda)\|\varphi\|_{L^{\infty}(B)}+\|\nabla \varphi\|_{L^{\infty}(B)} \\
& \lesssim(1+\lambda) P_{1}(\lambda)\|\varphi\|_{L^{2}(B)}+\lambda P_{2}(\lambda)\|\varphi\|_{L^{2}(B)} \\
& \lesssim\left((1+\lambda) P_{1}(\lambda)+\lambda P_{2}(\lambda)\right)\|\varphi\|_{L^{2}(B)}
\end{aligned}
$$

by using (3.4.2). Combining (3.5.25) with (3.5.26) and (3.5.4) we get

$$
\|\nabla \varphi(x)-\nabla \varphi(y)\| \lesssim P_{3}(\lambda)\|\varphi\|_{L^{2}(B)}|x-y|^{\alpha} .
$$

Proof of Lemma 3.2.1. This follows from Lemma 20 in [23] together with Proposition 3.4.1; we have $\mathscr{C}^{1+\alpha}$ functions which are close in $L^{2}(B)$. Hence, they are also close in $L^{\infty}(B)$, i.e. equation (3.2.1) holds and so does (3.2.2).

3.5.3. Heat kernel estimates. This subsection makes no assumptions on the finiteness of the volume of $\mathscr{M}$ and the existence of $C_{\text {count }}$ for the manifold $\mathscr{M}$. It will however use these properties for a manifold ball.

We fix a ball $B=B_{R}(x)$ for which we estimate the heat kernel $\tilde{K}^{B}$ by comparing it to $K^{B}$. Suppose that $\left\{\xi_{j}\right\}$ is an orthonormal basis for $L^{2}(\tilde{B})$ (with manifold measure). In this section all constants subsumed in $\lesssim, \gtrsim$ and $\sim$ will in general depend on $d, c_{\min }, c_{\max },\|g\|_{\alpha}, \alpha$.

Lemma 3.5.9. Let $A_{1}>1$ and a sufficiently small $\eta_{0}=\eta_{0}\left(A_{1}\right)>0$ be given. Assume $\epsilon_{0}$ is sufficiently small (depending on $\eta_{0}, A_{1}$, as well as the usual $d, c_{\min }, c_{\max }$, $\left.\|g\|_{\alpha}, \alpha\right)$, and $\left|g^{i k}(x)-\delta^{i k}\right|<\epsilon_{0}$. For $y \in B_{\frac{R}{2}}(x) \subset \Omega$, with $|x-y|^{2} \lesssim t \sim R^{2} \leq 1$ in a similar fashion to Assumption A.1, we have

$$
\sum_{\mu_{i} \leq \frac{A_{1}}{t}} \xi_{i}(x) \xi_{i}(y) e^{-\mu_{i} t} \sim_{\eta_{0}, A_{1}, d, c_{\max }, c_{\min },\|g\|_{\alpha}, \alpha} \sum_{\tilde{\mu}_{i} \leq \frac{A_{1}}{t}} \tilde{\xi}_{i}(x) \tilde{\xi}_{i}(y) e^{-\tilde{\mu}_{i} t} .
$$

If, in addition, we also have $|x-y|^{2} \sim t$, then

$$
\begin{aligned}
& \left|\sum_{\mu_{i} \leq \frac{A_{1}}{t}} \xi_{i}(x) \nabla \xi_{i}(y) e^{-\mu_{i} t}-\sum_{\tilde{\mu}_{i} \leq \frac{A_{1}}{t}} \tilde{\xi}_{i}(x) \nabla \tilde{\xi}_{i}(y) e^{-\tilde{\mu}_{i} t}\right| \\
& \lesssim_{A_{1}, d, c_{\max }, c_{\min },\|g\|_{\alpha}, \alpha} \eta_{0} \cdot \frac{R}{t} t^{\frac{-d}{2}} .
\end{aligned}
$$

The constants in (3.5.28) go to 1 as $\eta_{0} \rightarrow 0$.

Proof. We apply Lemma 3.5.5 with $J=\#\left\{j: \mu_{i} \leq A_{1} / t\right\} \leq\left(\frac{A_{1}}{t}\right)^{\frac{d}{2}} R^{d} \sim A_{1}^{\frac{d}{2}}$ and with $\eta<\eta_{0}$. Let $\epsilon_{0}$ be as guaranteed by Lemma 3.5.5. Since $\xi_{i}$ 's and $\tilde{\xi}_{i}$ 's are $L^{2}$-normalized, Lemma 3.5.3 and 3.5.5 implies for $\mu_{i} \leq \frac{A_{1}}{t}$

$$
\begin{aligned}
& \left|\xi_{i}(x) \xi_{i}(y) e^{-\mu_{i} t}-\tilde{\xi}_{i}(x) \tilde{\xi}_{i}(y) e^{-\tilde{\mu}_{i} t}\right| \\
& \leq\left|\xi_{i}(x)-\tilde{\xi}_{i}(x)\left\|\xi_{i}(y)\left|e^{-\mu_{i} t}+\right| \xi_{i}(y)-\tilde{\xi}_{i}(y)\right\| \xi_{i}(x)\right| e^{-\mu_{i} t}+\left|\xi_{i}(x)\left\|\xi_{i}(y)\right\| e^{-\mu_{i} t}-e^{-\tilde{\mu}_{i} t}\right| \\
& \lesssim Q_{1}\left(A_{1} t^{-1} R^{2}\right) \eta\left(\left|\xi_{i}(y)\right| e^{-\mu_{i} t}+\left|\xi_{i}(x)\right| e^{-\mu_{i} t}\right)+\left|\xi_{i}(x) \| \xi_{i}(y)\right| t \eta \mu_{i} e^{-\mu_{i} t} \lesssim A_{1}^{3 \beta_{\mathrm{loc}}+1} \eta .
\end{aligned}
$$


Using Weyl's Lemma (Lemma 3.4.2) for the ball with Dirichlet boundary conditions (see Lemma 3.4.2), we have

$$
\begin{aligned}
\left\|\sum_{\mu_{i} \leq \frac{A_{1}}{t}} \xi_{i}(x) \xi_{i}(y) e^{-\mu_{i} t}-\sum_{\tilde{\mu}_{i} \leq \frac{A_{1}}{t}} \tilde{\xi}_{i}(x) \tilde{\xi}_{i}(y) e^{-\tilde{\mu}_{i} t}\right\| & \lesssim A_{1}^{3 \beta_{\mathrm{loc}}}\left(1+A_{1}\right) \eta J \\
& \lesssim A_{1}^{3 \beta_{\mathrm{loc}}+1} \eta \sum_{\mu_{i} \leq \frac{A_{1}}{t}} \xi_{i}(x) \xi_{i}(y) e^{-\mu_{i} t}
\end{aligned}
$$

by the (Euclidean) estimates in the proof of Lemma 3.1.4 and since $R \lesssim 1$. We obtain the desired estimate (3.5.28) by taking $\eta_{0}$ sufficiently small. Similarly,

$$
\begin{aligned}
& \left|\xi_{i}(x) \nabla \xi_{i}(y) e^{-\mu_{i} t}-\tilde{\xi}_{i}(x) \nabla \tilde{\xi}_{i}(y) e^{-\tilde{\mu}_{i} t}\right| \\
& \leq\left|\xi_{i}(x)-\tilde{\xi}_{i}(x)\left\|\nabla \xi_{i}(y)\left|e^{-\mu_{i} t}+\right| \nabla \xi_{i}(y)-\nabla \tilde{\xi}_{i}(y)\right\| \xi_{i}(x)\right| e^{-\mu_{i} t} \\
& \quad+\left|\xi_{i}(x)\left\|\nabla \xi_{i}(y)\right\| e^{-\mu_{i} t}-e^{-\tilde{\mu}_{i} t}\right| \\
& \lesssim \\
& \quad+\left(\left(A_{1} t^{-1} R^{2}\right)^{\beta_{\mathrm{loc}}}\left|\nabla \xi_{i}(y)\right| e^{-\mu_{i} t}+\left(A_{1} t^{-1} R^{2}\right)^{\beta_{\mathrm{loc}}+1} r^{-1}\left|\xi_{i}(x)\right| e^{-\mu_{i} t}\right) \\
& \quad+\left|\xi_{i}(x) \| \nabla \xi_{i}(y)\right| t \eta \mu_{i} e^{-\mu_{i} t} \\
& \lesssim A_{1}^{3 \beta_{\mathrm{loc}}+2} \eta R^{-1} .
\end{aligned}
$$

Thus, equation (3.5.29) also clearly follows by $\eta_{0}$ sufficiently small.

Lemma 3.5.10. Let $\eta_{0}>0$ be given and assumed to be sufficiently small. Assume $\epsilon_{0}$ is sufficiently small (depending on $\eta_{0}$, as well as the usual $d, c_{\min }, c_{\max }$, $\|g\|_{\alpha}, \alpha$ ), and $\left|g^{i k}(x)-\delta^{i k}\right|<\epsilon_{0}$. For $y \in B_{\frac{R}{2}}(x) \subset \Omega$ with $|x-y|^{2} \lesssim t \sim R^{2} \leq 1$ (in a similar fashion to Assumption A.1) and $s \leq t$,

$$
\begin{aligned}
& \tilde{K}_{t}^{B}(x, y) \sim_{\eta_{0}, d, c_{\min }, c_{\max },\|g\|_{\alpha}, \alpha} K_{t}^{B}(x, y),
\end{aligned}
$$

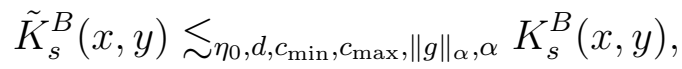

and

$$
\left\|\nabla \tilde{K}_{s}^{B}(x, y)\right\| \lesssim \eta_{0}, d, c_{\min }, c_{\max },\|g\|_{\alpha}, \alpha \frac{R}{s}\left(s R^{-2}\right)^{-2 \beta_{\mathrm{loc}}-1} s^{\frac{-d}{2}} .
$$

If, in addition, we have $|x-y|^{2} \sim t$, then

$$
\left\|\nabla \tilde{K}_{t}^{B}(x, y)-\nabla K_{t}^{B}(x, y)\right\| \lesssim_{d, c_{\min }, c_{\max },\|g\|_{\alpha}, \alpha} \eta_{0} \cdot \frac{R}{t} t^{\frac{-d}{2}} .
$$

The constants in (3.5.30) go to 1 as $\eta_{0} \rightarrow 0$.

Proof. We estimate the tail:

$$
\begin{aligned}
\left\|\sum_{\mu_{i} \geq \frac{A_{1}}{t}} \xi_{i}(x) \xi_{i}(y) e^{-\mu_{i} t}\right\| & \leq e^{-\frac{1}{2} A_{1}}\left\|\sum_{\mu_{i} \geq \frac{A_{1}}{t}} \xi_{i}(x) \xi_{i}(y) e^{-\frac{1}{2} \mu_{i} t}\right\| \\
& \leq e^{-\frac{1}{2} A_{1}} \tilde{K}_{\frac{1}{4} t}^{B}(x, x) \tilde{K}_{\frac{1}{4} t}^{B}(y, y) \underbrace{\lesssim}_{\text {using [16] }} e^{-\frac{1}{2} A_{1}} t^{-\frac{d}{2}} .
\end{aligned}
$$


This, combined with (3.5.28), for $A_{1}$ large enough, gives (3.5.30). From [15] we also get (3.5.31). We also have

$$
\begin{aligned}
\left\|\nabla_{x} \sum_{\tilde{\mu} \geq \frac{A_{1}}{s}} \tilde{\xi}_{i}(x) \tilde{\xi}_{i}(y) e^{-\tilde{\mu}_{i} s}\right\| & =\left\|\int_{B} \nabla_{x} \tilde{G}^{B}(x, w) \sum_{\tilde{\mu} \geq \frac{A_{1}}{s}} \tilde{\Delta}^{B} \tilde{\xi}_{i}(w) \tilde{\xi}_{i}(y) e^{-\tilde{\mu}_{i} s}\right\| \\
& =\left\|\int_{B} \nabla_{x} \tilde{G}^{B}(x, w) \sum_{\tilde{\mu} \geq \frac{A_{1}}{s}} \tilde{\mu}_{i} \tilde{\xi}_{i}(w) \tilde{\xi}_{i}(y) e^{-\tilde{\mu}_{i} s}\right\| \\
& =s^{-1}\left\|\int_{B} \nabla_{x} \tilde{G}^{B}(x, w) \sum_{\tilde{\mu} \geq \frac{A_{1}}{s}} \tilde{\xi}_{i}(w) \tilde{\xi}_{i}(y)\left(\tilde{\mu}_{i} s e^{-\tilde{\mu}_{i} s}\right)\right\| \\
& \lesssim s^{-1} \int_{B}\left\|\nabla_{x} \tilde{G}^{B}(x, w)\right\| \sum_{\tilde{\mu} \geq \frac{A_{1}}{s}}\left|\tilde{\xi}_{i}(w) \| \tilde{\xi}_{i}(y)\right| e^{-\frac{1}{2} \tilde{\mu}_{i} s} \\
& \lesssim e^{-\frac{1}{4} A_{1}} s^{-1} \int_{B}\left\|\nabla_{x} \tilde{G}^{B}(x, w)\right\| \tilde{K}_{s / 8}^{B}(w, w)^{\frac{1}{2}} \tilde{K}_{s / 8}^{B}(y, y)^{\frac{1}{2}} \\
& \lesssim e^{-\frac{1}{4} A_{1}} s^{-\frac{d}{2}-1} \int_{B}\left\|\nabla_{x} \tilde{G}^{B}(x, w)\right\| \lesssim e^{-\frac{1}{4} A_{1}} s^{-\frac{d}{2}-1} R,
\end{aligned}
$$

since by (3.5.6) we have $\left\|\nabla \tilde{G}^{B}(x, \cdot)\right\|_{L^{1}\left(B_{R}\right)} \lesssim R$. If we now take $s=t$ then, by the Euclidean estimates and (3.5.29), for $A_{1}$ large enough, we obtain both the lower and upper bounds (3.5.33).

To prove estimate (3.5.32), we use the above estimate and notice that we also have (from Lemma 3.5.3 and Weyl's Lemma for the ball with Dirichlet boundary conditions)

$$
\begin{aligned}
\left\|\sum_{\tilde{\mu} \leq \frac{A_{1}}{s}} \nabla \tilde{\xi}_{i}(x) \tilde{\xi}_{i}(y) e^{-\tilde{\mu}_{i} s}\right\| & \lesssim R^{-d} \sum_{\tilde{\mu} \leq \frac{A_{1}}{s}} \tilde{\mu}_{i} R\left(\tilde{\mu}_{i} R^{2}\right)^{2 \beta_{\mathrm{loc}}+1} e^{-\tilde{\mu}_{i} s} \\
& =R^{-d}\left(s R^{-2}\right)^{-2 \beta_{\mathrm{loc}}-2} R^{-1} \sum_{\tilde{\mu} \leq \frac{A_{1}}{s}}\left(\tilde{\mu}_{i} s\right)^{2 \beta_{\mathrm{loc}}+2} e^{-\tilde{\mu}_{i} s} \\
& \lesssim R^{-d}\left(s R^{-2}\right)^{-2 \beta_{\mathrm{loc}}-2} R^{-1} \sum_{\tilde{\mu} \leq \frac{A_{1}}{s}} 1 \\
& \lesssim A_{1}^{\frac{d}{2}} R^{-1}\left(s R^{-2}\right)^{-2 \beta_{\mathrm{loc}}-2} s^{\frac{-d}{2}} \\
& \lesssim \frac{R}{s}\left(s R^{-2}\right)^{-2 \beta_{\mathrm{loc}}-1} s^{\frac{-d}{2}} .
\end{aligned}
$$

Lemma 3.5.10 will be used to get Proposition 3.1.2 for the case of a manifold. We will need to improve estimate (3.5.32), which in turn requires the following: 
Lemma 3.5.11. Let $|y|<\frac{R}{4}, r<\frac{R}{4}$ and $s^{\frac{1}{2}} \leq r$. Let $B^{y}\left(s^{\prime}\right)$ be Brownian motion started at $y$. Then

$$
P\left(\sup _{0 \leq s^{\prime} \leq s}\left|B^{y}\left(s^{\prime}\right)\right|>|y|+r\right) \lesssim d, c_{\min }, c_{\max } e^{-c^{\prime}\left(d, c_{\min }, c_{\max }\right) \frac{r}{\sqrt{s}}} .
$$

Proof. This follows from Lemma 3.3.4.

Proof of Proposition 3.1.2; case of $B_{R}(z)$ with metric at least $\mathscr{C}^{2}$. By rescaling we may assume that $R \leq 1$. We upper bound $\delta_{0}$ so that $\left|g^{i k}(x)-\delta^{i k}\right|<\epsilon_{0}$, where $\epsilon_{0}$ is as prescribed by Lemma 3.5.10 (this is done as in (3.1.16)).

Estimates (3.1.1) and the first part of (3.1.4) follow from the Euclidean case and estimates (3.5.30) and (3.5.31). Estimate (3.1.2) and estimate (3.1.3) follow from (3.5.33) and Euclidean ball estimates.

We now turn to the second and third parts in (3.1.4). Without loss of generality we identify $z=0$. Let $a$ be such that $a \sum_{j=1}^{\infty} \frac{1}{j^{2}}=\frac{1}{4}$. Define stopping times $\tau_{1}, \tau_{2}, \ldots$ by

$$
\tau_{n}=\inf \left\{s^{\prime}:\left|B^{z}\left(s^{\prime}\right)\right|=a R \sum_{j=1}^{n} \frac{1}{j^{2}}\right\} .
$$

For $n>1$, define the set of paths

$$
B_{n}=\left\{\omega \in \Omega: \tau_{n}(\omega) \leq\left(1-2^{-n}\right) s\right\} .
$$

For $n>1$ define $G_{n} \subset B_{n}$ as

$$
G_{n}=B_{n} \backslash B_{n-1}
$$

and

$$
G_{1}=B_{1}
$$

We estimate using Lemma 3.5.11:

$$
P\left(G_{1}\right) \leq \exp \left(-c^{\prime} \frac{a}{1^{2}} R s^{-\frac{1}{2}}\right)
$$

and for $n>1$

$$
P\left(G_{n}\right) \leq \exp \left(-c^{\prime} \frac{a 2^{\frac{n-1}{2}}}{n^{2}} R s^{-\frac{1}{2}}\right) .
$$

We need another lemma: 0 .

Lemma 3.5.12. The set $\left\{\omega \in \Omega: \tau_{n} \leq s \forall(n \geq 1), \omega \notin \cup G_{n}\right\}$ has probability

Proof. Now

$$
\begin{aligned}
\left\{\omega \in \Omega: \tau_{n} \leq s \forall(n \geq 1), \omega \notin \cup G_{n}\right\} & =\left\{\omega \in \Omega: \tau_{n} \leq s \forall(n \geq 1), \omega \notin \cup B_{n}\right\} \\
& =\left\{\omega \in \Omega: s \geq \tau_{n} \geq\left(1-2^{-n}\right) s \forall(n>1)\right\} \\
& \subset\left\{\omega \in \Omega: \tau_{n}-\tau_{n-1} \leq 2^{-n} s \forall(n>1)\right\} .
\end{aligned}
$$

However, the set $\left\{\omega \in \Omega: \tau_{n}-\tau_{n-1} \leq 2^{-n} s\right\}$ has probability decaying superexponentially in $n$ by Lemma 3.5.11.

We now continue with the proof of Proposition 3.1.2; case of $B_{R}(z)$ with metric at least $\mathscr{C}^{2}$. Define $H_{n}=G_{n} \backslash\left(\cup_{1}^{n-1} G_{i}\right)$. We now have a disjoint partition of 
$\left\{\omega \in \Omega: \tau_{n} \leq s \forall(n \geq 1)\right\}$ (up to measure 0) by the collection $\left\{H_{i}\right\}$. Set $\tilde{K}_{s}^{D}(\cdot, \cdot):=$ $\tilde{K}_{s}^{B_{R}(z)}(\cdot, \cdot)$. For $|y|>\frac{R}{4}$ we have

$$
\tilde{K}_{s}^{D}\left(z, y_{0}\right)=\sum_{n=1}^{\infty} \mathbf{E}_{\omega}\left(\chi_{H_{n}} \tilde{K}_{s-\tau_{n}}^{D}\left(B^{z}\left(\tau_{n}\right), y\right)\right) .
$$

Taking gradient and using equation (3.5.32) we get

$$
\begin{aligned}
& \left|\nabla_{x} \tilde{K}_{s}^{D}(z, y)\right|=\left|\sum_{n=1}^{\infty} \mathbf{E}_{\omega}\left(\chi_{H_{n}} \nabla_{x} \tilde{K}_{s-\tau_{n}}^{D}\left(B^{z}\left(\tau_{n}\right), y\right)\right)\right| \\
& \lesssim_{d, c_{\min }, c_{\max },\|g\|_{\alpha}, \alpha} \sum_{n=1}^{\infty} \frac{R}{s}\left(2^{-n} s R^{-2}\right)^{-2 \beta_{\mathrm{loc}}-1} s^{\frac{-d}{2}} P\left(H_{n}\right) \\
& \lesssim_{d, c_{\min }, c_{\max },\|g\|_{\alpha}, \alpha} \sum_{n=1}^{\infty} \frac{R}{s}\left(2^{-n / 2} s^{\frac{1}{2}} R^{-1}\right)^{-4 \beta_{\mathrm{loc}}-2} s^{\frac{-d}{2}} \exp \left(-c^{\prime} \frac{a 2^{-1 / 2}}{n^{2}} 2^{n / 2} s^{-\frac{1}{2}} R\right) \\
& \lesssim_{d, c_{\min }, c_{\max },\|g\|_{\alpha}, \alpha} \sum_{n=1}^{\infty} \frac{R}{t}\left(2^{-n / 2} t^{\frac{1}{2}} R^{-1}\right)^{-4 \beta_{\mathrm{loc}}-2} t^{\frac{-d}{2}} \exp \left(-c^{\prime} \frac{a 2^{-1 / 2}}{n^{2}} 2^{n / 2} t^{-\frac{1}{2}} R\right) \\
& \leq \frac{R}{t} t^{\frac{-d}{2}} \sum_{n=1}^{\infty}\left(2^{-n / 2} t^{\frac{1}{2}} R^{-1}\right)^{-4 \beta_{\mathrm{loc}}-2} \exp \left(-c^{\prime} \frac{a 2^{-1 / 2}}{n^{2}} 2^{n / 2} t^{-\frac{1}{2}} R\right) \\
& \lesssim_{d, c_{\min }, c_{\max },\|g\|_{\alpha}, \alpha} \frac{R}{t} t^{\frac{-d}{2}},
\end{aligned}
$$

where we may replace $s$ with $t$ above, since $s \leq t$, and each of the summands is increasing in $s$ as long as it is sufficiently small with respect to $R^{2}$ (independently of $n$ when $n>1)$. This proves the second and third parts of (3.1.4) for $B_{R}(z)$ with metric at least $\mathscr{C}^{2}$.

Remark 3.5.13. The proof below makes no assumption on the volume of $\mathscr{M}$, and works for the case of $\mathscr{M}$ having infinite volume as well.

Proof of Proposition 3.1.2 for the heat kernel of $\mathscr{M}$, with metric at least $\mathscr{C}^{2}$. As for the Neumann heat kernel, the starting point is Proposition 3.3.2, which allows us to localize. We use Proposition 3.1.2 for the ball $B_{2 \delta_{0} R_{z}}(z)$ with metric at least $\mathscr{C}^{2}$. For this proof, we denote by $C_{2}[B]$ be the $C_{2}$ constant for the Dirichlet ball case, and set $K_{s}^{D}(\cdot, \cdot):=K_{s}^{2 \delta_{0} R_{z}}(\cdot, \cdot)$, the heat kernel for the ball $B\left(z, 2 \delta_{0} R_{z}\right)$ with Dirichlet boundary conditions. For $s \leq t$,

$$
\begin{aligned}
& \left|K_{s}(x, y)-\tilde{K}_{s}^{D}(x, y)\right|=\left|\sum_{n=1}^{+\infty} \mathbf{E}_{\omega}\left[\tilde{K}_{s-\tau_{n}}^{D}\left(x_{n}(\omega), y\right) \mid \tau_{n}<s\right] P_{\omega}\left(\tau_{n}(\omega)<s\right)\right| \\
& \lesssim_{C_{2}[B]} \sum_{n=1}^{\infty} t^{-\frac{d}{2}} \underbrace{e^{-n \frac{\left(\frac{\delta_{0} R_{z}}{2}\right)^{2}}{M s}}}_{\text {eqn. }(3.3 .10)} \lesssim_{C_{2}[B], \delta_{0}, \delta_{1}} t^{-\frac{d}{2}} e^{-\frac{\left(\frac{\delta_{0} R z}{2}\right)^{2}}{M s}} .
\end{aligned}
$$

This proves (3.1.1) and the first part of (3.1.4) (see Remark 3.3.3). For the gradient estimates, i.e. (3.1.2), (3.1.3), and the second and third part of (3.1.4), 


$$
\begin{aligned}
\left\|\nabla_{y} K_{s}(x, y)-\nabla_{y} \tilde{K}_{s}^{D}(x, y)\right\| & \leq \sum_{n=1}^{+\infty}\left\|\nabla_{y} \mathbf{E}_{\omega}\left[\tilde{K}_{s-\tau_{n}}^{D}\left(x_{n}(\omega), y\right) \mid \tau_{n}<s\right]\right\| P_{\omega}\left(\tau_{n}(\omega)<s\right) \\
& \lesssim_{2}^{\prime}[B] \sum_{n=1}^{\infty} t^{-\frac{d}{2}} \frac{\delta_{0} R_{z}}{t} \underbrace{e^{-n \frac{\left(\frac{\delta_{0} R_{z}}{2}\right)^{2}}{M s}}}_{\text {eqn. }(3.3 .10)} \\
& \lesssim_{C_{2}^{\prime}[B], \delta_{0}, \delta_{1}} t^{-\frac{d}{2}} \frac{\delta_{0} R_{z}}{t} e^{-\frac{\left(\frac{\delta_{0} R_{z}}{2}\right)^{2}}{M s}}
\end{aligned}
$$

giving us $C_{9}$. By Remark 3.3.3 the exponential term from equation (3.3.10) can be made small enough so that we obtain estimate (3.1.2) as well as the second and third parts of (3.1.4).

\section{The proof of Theorem 2.2.8}

We remind the reader of Remark 3.1.1 which notes that the proof of Proposition 3.1.2 for the heat kernel of $\mathscr{M}$, with metric at least $\mathscr{C}^{2}$ (appearing at the end of section 3.5.3), made no assumptions on the finiteness of the volume of $\mathscr{M}$ and the existence of $C_{\text {count }}$.

4.1. The case $g \in \mathscr{C}^{2}$. We appropriately choose heat kernels $\left\{K_{t}\left(z, y_{i}\right)\right\}_{i=1, \ldots, d}$, with $t \sim R_{z}^{2}$, that provide a local coordinate chart with the properties claimed in the Theorem 2.2.8:

Proof of Theorem 2.2.8 for $g \in \mathscr{C}^{2}$. Without loss of generality we may assume $\rho=R_{z}=1$, and thus, by Remark 3.1.1, we may apply Proposition 3.1.2. Let us consider the Jacobian $\tilde{J}(x)$, for $x \in B_{c_{1} R_{z}}(z)$, of the map

$$
\tilde{\Phi}:=R_{z}^{-d} t^{d / 2}\left(t / R_{z}^{2}\right) \Phi .
$$

By (3.1.3) we have $\left|\tilde{J}_{i j}(x)-C_{2}^{\prime}\left\langle p_{i}, \frac{x-y_{j}}{\left\|x-y_{j}\right\|}\right\rangle R_{z}^{-1}\right| \leq C_{9} R_{z}^{-1}$. As dictated by Proposition 3.1 .2 , by choosing $\delta_{0}, \delta_{1}$ appropriately (and, correspondingly, $c_{1}$ and $c_{6}$ ), we can make the constant $C_{9}$ smaller than any chosen $\epsilon$, for all entries, and for all $x$ at distance no greater than $c_{1} R_{z}$ from $z$, where we use $t=t_{z}=c_{6} R_{z}^{2}$ for $\tilde{\Phi}$. Therefore for $c_{1}$ small enough compared to $c_{4}$ we can write $R_{z} \tilde{J}(x)=G_{d}+E(x)$ where $G_{d}$ is the Gramian matrix $\left\langle p_{i}, p_{j}\right\rangle$ (indepedent of $x$ !), and $\left|E_{i j}(x)\right|<\epsilon$, for $x \in B_{c_{1} R_{z}}(z)$. This implies that $R_{z}^{-1}\left(\sigma_{\min }-C_{d} \epsilon\right)\|v\| \leq\|\tilde{J}(x) v\| \leq R_{z}^{-1}\left(\sigma_{\max }+C_{d} \epsilon\right)\|v\|$, with $C_{d}$ depending linearly on $d$, where $\sigma_{\max }$ and $\sigma_{\min }$ are the largest and, respectively, smallest eigenvalues of $G_{d}$. At this point we choose $\epsilon$ small enough, so that the above bounds imply that the Jacobian is essentially constant in $B_{c_{1} R_{z}}(z)$, and by integrating along a path from $x_{1}$ to $x_{2}$ in $B_{c_{1} R_{z}}(z)$, we obtain the Theorem ( $\Phi$ and $\tilde{\Phi}$ differ only by scalar multiplication). We note that $\epsilon \sim \frac{1}{d}$ suffices.

We discuss the proof for $g \in \mathscr{C}^{\alpha}$, and $\mathscr{M}$ has possibly infinite volume in Section 4.2. Such proof is based on approximation arguments via heat kernels corresponding to smooth metrics on finite volume submanifolds.

4.2. The case $g \in \mathscr{C}^{\alpha}$. In this section we discuss heat kernel estimates and the heat kernel triangulation Theorem in the case when $\mathscr{C}^{\alpha}$. The key ingredient for the 
proof of Theorem 2.2.8 for the case of $g \in \mathscr{C}^{\alpha}$, are the heat kernel estimates similar to those of Proposition 3.1.2.

Before we turn to the proof of Theorem 2.2.8 for the case $g \in \mathscr{C}^{\alpha}$, we need one more statement about the case $g \in \mathscr{C}^{2}$. Consider the following variant of Proposition 3.3.2.

Proposition 4.2.1. (Variant of Proposition 3.3.2) Assume $g \in \mathscr{C}^{2}$. Let $w \in \Omega$ and $R_{w} \leq \operatorname{dist}(w, \partial \Omega)$, or $w \in \mathscr{M}$ and $R_{w} \leq r_{U}(w)$. Let $z$ and $R_{z}$ be similarly defined. Assume $z \notin B_{R_{w}}(w)$, and $w \notin B_{R_{z}}(z)$. For each path $B_{\omega}^{z}$ (starting at $z)$, we define $\tau_{1}(\omega) \leq \tau_{2}(\omega) \leq \ldots$ as follows. Let $\tau_{1}(\omega)$ be the first time that $B_{\omega}^{z}$ enters $B\left(w, \frac{3}{4} R_{w}\right)$ (if this does not happen, let $\tau_{1}(\omega)=+\infty$ ). Let $z_{1}=B_{\omega}^{z}\left(\tau_{1}\right)$. By induction, for $n>1$ let $\tau_{n}(\omega)$ be the first time after $\tau_{n-1}(\omega)$ that $B_{\omega}^{z}$ re-enters $B\left(w, \frac{3}{4} R_{w}\right)$ after having exited $B\left(w, \frac{1}{2} R_{w}\right)$, or $+\infty$ otherwise. Let $z_{n}(\omega)=B_{\omega}^{z}\left(\tau_{n}\right)$. If $\tau_{n}(\omega)=+\infty$, let $\tau_{n+k}(\omega)=+\infty$ for all $k \geq 0$. Then

$$
K_{s}(z, w)=\sum_{n=1}^{+\infty} \mathbf{E}_{\omega}\left[K_{s-\tau_{n}(\omega)}^{D}\left(z_{n}(\omega), w\right) \mid \tau_{n}<s\right] P_{\omega}\left(\tau_{n}<s\right)
$$

where

$$
K_{s}^{D}=K_{s}^{\operatorname{Dir}\left(B_{\frac{1}{2} R_{w}}(w)\right)} .
$$

Moreover, there exists an $M=M\left(c_{\min }, c_{\max }\right)$ such that

$$
P\left(\tau_{n}<s\right) \lesssim_{d, M, c_{\min }, c_{\max }} \exp \left\{-(n-1)\left(\frac{R_{w}}{8}\right)^{2}(2 M s)^{-1}-\left(\frac{R_{z}}{8}\right)^{2}(2 M s)^{-1}\right\}
$$

The proof of this Proposition is along the same lines as that of Proposition 3.3.2.

Proof of Theorem 2.2.8 for $g \in \mathscr{C}^{\alpha}(\mathscr{M})$ with $|\mathscr{M}| \leq \infty$. Consider a sequence of metrics $\left\{g_{k}\right\} \subseteq \mathscr{C}^{2}(\mathscr{M})$, with increasing compact supports $\left\{\mathscr{M}_{k}\right\}$, converging to $g$ in $\mathscr{C}^{\alpha}$ (and therefore bounded in $\mathscr{C}^{\alpha}$ ), and such that $g_{k}$ is uniformly elliptic with constants $\frac{1}{2} c_{\min }, 2 c_{\max }$ (which is possible since $c_{\min }$ and $c_{\max }$ are continuous functions of the components of the metric tensor). Let $K_{k}$ be the heat kernel associated with $g_{k}$. Note that for this heat kernel and its gradient we have bounds, from above with constants uniform in $k$ for any fixed compact $\mathscr{E}$ away from $\partial \mathscr{M}$. We proceed as in the proof of Theorem II.3.1 in [49]. The key ingredients are uniform (in $k$, for a fixed compact) upper bounds on $K_{k}$ (which follow from Propositions 3.3 .2 and 4.2.1), and that $\left\{K_{k}\right\}$ is equicontinuous, which follows from the uniform upper bounds on the gradient of $K_{k}$ (for a fixed compact we have uniform lower bounds on $R_{z}$ and $R_{w}$ and estimate (4.2.2)). It could also made follow from Stroock's paper (NashMoser estimates that say the $K_{k}$ is Hölder of order and with constants depending only the ellipticity constants)). The proof of Theorem II.3.1 in [49] then implies that $K_{k} \rightarrow K_{\mathscr{M}}$ as $k \rightarrow+\infty$, uniformly on compacts. Therefore the uniform (in $k$ ) bi-Lipschitz bounds on the map

$$
x \rightarrow\left(K_{k, t}\left(x, y_{1}\right), \ldots, K_{k, t}\left(x, y_{d}\right)\right)
$$

on $B_{R}(z)$, imply the same bounds for

$$
x \rightarrow\left(K_{\mathscr{M}, t}\left(x, y_{1}\right), \ldots, K_{\mathscr{M}, t}\left(x, y_{d}\right)\right) .
$$




\section{Examples}

5.1. Localized eigenfunctions. The following example shows that the factors $\gamma_{1}, \ldots, \gamma_{d}$ in Theorems 2.1.1 and 2.2.1 may in fact be required to be as small as $R_{z}^{\frac{d}{2}}$.
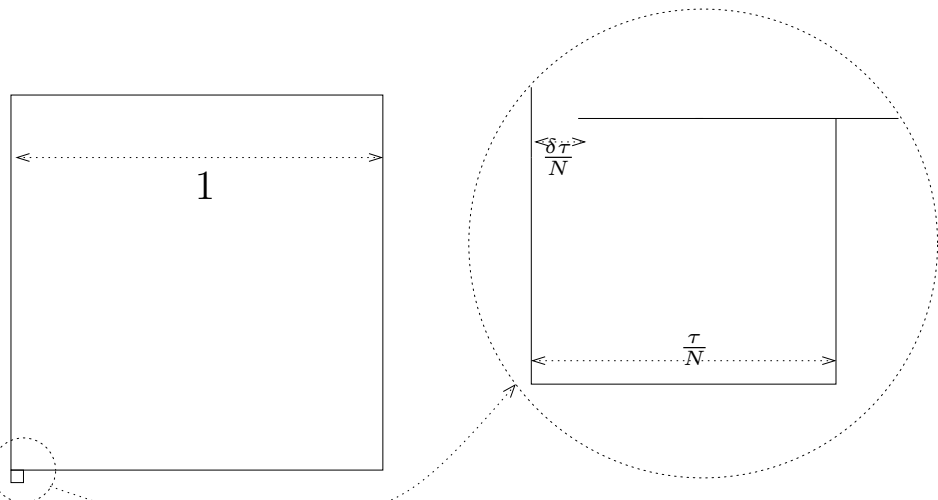

Figure 1. Example of localization.

Let $\tau$ below be the golden ratio. Consider the domain $\Omega_{\delta}$ as in Figure 1, with Dirichlet boundary conditions. We will let $z$ to be the center of the small square. Let $\lambda_{j}^{\delta}$ and $\varphi_{j}^{\delta}$ be the eigenvalues and eigenfunctions on $\Omega_{\delta}$. Fix $A>C N$. Let $\Lambda_{A}^{\delta}=\left\{j: \lambda_{j}^{\delta} \leq A^{2}\right\}$. The cardinality of $\Lambda_{A}^{\delta}$ is uniformly bounded above by Weyl Lemma. It is also bounded below, since in this case we can easily obtain a reverse Weyl Lemma. To see this, let $\left(\partial \Omega_{\delta}\right)_{A}=\{x \in \Omega: d(x, \partial \Omega) \leq a / A\}$, so that the heat kernel estimates in Lemma 3.1.4 hold for $t=b A^{-2}$, and observe that

$$
\begin{aligned}
\#\left\{j: \lambda_{j}^{\delta} \leq A^{2}\right\} & \geq e^{+b} \int_{\Omega_{\delta} \backslash\left(\partial \Omega_{\delta}\right)_{A}} \sum_{\lambda_{j}^{\delta} \leq A}\left|\varphi_{j}^{\delta}(x)\right|^{2} e^{-\lambda_{j}^{\delta} t} \\
& \geq e^{+b}\left(\int_{\Omega_{\delta} \backslash\left(\partial \Omega_{\delta}\right)_{A}} K_{t}^{\delta}(x, x)-\int_{\Omega_{\delta} \backslash\left(\partial \Omega_{\delta}\right)_{A}} K_{t / 2}^{\delta}(x, x) e^{-\frac{A^{2} t}{2}}\right) \\
& \gtrsim e^{+b}\left(\frac{1}{2}-\frac{a}{A}\right)^{2}\left(C_{1} t^{-1}-C_{2} e^{-\frac{b}{2}} t^{-1}\right) \gtrsim e^{+b} b^{-1} A^{2},
\end{aligned}
$$

where we choose $b$ so that the last inequality holds, and thus determine $a$ and $A$, which are chosen so that Lemma 3.1.4 and Proposition 3.1.2 hold.

Fix $j=1$. The sequence $\left\{\lambda_{j}^{\delta}\right\}_{\delta>0}$ is bounded and hence the families $\left\{\varphi_{j}^{\delta}\right\}_{\delta>0}$ and $\left\{\nabla \varphi_{j}^{\delta}\right\}_{\delta \geq 0}$ are equicontinuous (by Proposition 3.4.1), therefore there exists a sequence $\delta_{k} \rightarrow 0$ such that $\varphi_{j}^{\delta_{k}} \rightarrow \varphi_{j}, \nabla \varphi_{j}^{\delta_{k}} \rightarrow \nabla \varphi_{j}$ and $\lambda_{j}^{\delta_{k}} \rightarrow \lambda_{j}$. We can repeat this argument for any $j \leq \liminf _{k} \#\left\{j: \lambda_{j}^{\delta_{k}} \leq A^{2}\right\}:=j_{\max }(A)$, which is strictly positive and tending to $+\infty$ as $A \rightarrow+\infty$, by the above. By a diagonal argument, we can find a subsequence $\delta_{l}$ such that for any $j \leq j_{\max }(A), \varphi_{j}^{\delta_{l}} \rightarrow \varphi_{j}, \nabla \varphi_{j}^{\delta_{l}} \rightarrow \nabla \varphi_{j}$. Let us look at some properties of $\varphi_{j}$. Clearly, $\varphi_{j}$ is an eigenfunction for $\Delta$ with Dirichlet boundary conditions on $\Omega_{0}$. Since $\left(\frac{\tau}{N} n_{1}\right)^{2}+\left(\frac{\tau}{N} n_{2}\right)^{2}$ is irrational for any $n_{1}, n_{2} \in \mathbf{Z}$, every $\varphi_{j}$ is supported in either the small square, or the big square. Recall that $z$ is the center of the small square. For any $j \leq j_{\max }(A)$ if $\varphi_{j}$ has support in $S_{0}$ then $\left\|\nabla \varphi_{j}\right\| \gtrsim(\tau / N)^{-2}$. Let $\delta_{l}$ be small enough so that $\left\|\nabla \varphi_{j}^{\delta_{l}}\right\| \gtrsim(\tau / N)^{-2}$, for all 
$j \leq j_{\max }(A)$. By choosing $A$ larger than $c_{5}(\tau / 2 N)$, where $c_{5}$ is as in Theorem 2.1.1, all possible eigenfunctions that may get chosen in the Theorem will correspond to $j \leq j_{\max }(A)$, and therefore the lower bound for the $\gamma_{i}$ is sharp.

See http://pmc.polytechnique.fr/pagesperso/dg/recherche/localization_e.htm for nice demonstrations of the above example.

\subsection{Non-simply connected domain.}
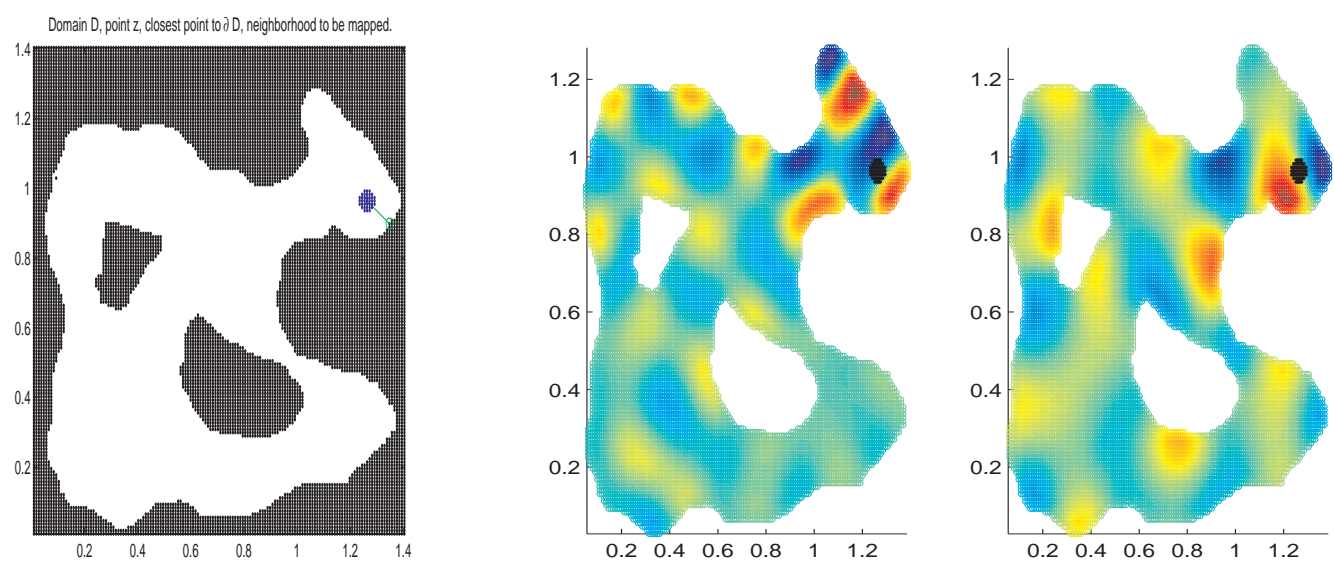

Figure 2. Top left: a non-simply connected domain in $\mathbf{R}^{2}$, and the point $z$ with its neighborhood to be mapped. Top right: the image of the neighborhood under the map. Bottom: Two eigenfunctions for mapping.

\section{References}

[1] Belkin, M., and P. Niyogi: Laplacian eigenmaps and spectral techniques for embedding and clustering. - Advances in Neural Information Processing Systems 14 (NIPS 2001), MIT Press, Cambridge, 2001, 585-591.

[2] Belkin, M., and P. Niyogi: Laplacian eigenmaps for dimensionality reduction and data representation. - Neural Computation 6:15, 2003, 1373-1396.

[3] Belkin, M., and P. Niyogi: Using manifold structure for partially labelled classification. Advances in Neural Information Processing Systems 15, 2003.

[4] Belkin, M., and P. Niyogi: Semi-supervised learning on Riemannian manifolds. - Machine Learning 56 (Invited Special Issue on Clustering), 2004, 209-239.

[5] Bérard, P. B., G. Besson, and S. Gallot: Embedding Riemannian manifolds by their heat kernel. - Geom. Funct. Anal. 4:4, 1994, 374-398.

[6] Chaplain, M., M. Ganesh, and I. Graham: Spatio-temporal pattern formation on spherical surfaces: numerical simulation and application to solid tumor growth. - J. Math. Biol. 42, 2001, $387-423$.

[7] Cheeger, J.: A lower bound for the smallest eigenvalue of the Laplacian. - In: Problems in Analysis, edited by R. C. Gunning, Princeton Univ. Press, 195-199.

[8] Chung, F. R. K.: Spectral graph theory. - CBMS Regional Conference Series in Mathematics 92, Published for the Conference Board of the Mathematical Sciences, Washington, DC, 1997.

[9] Coifman, R. R., I. G. Kevrekidis, S. Lafon, M. Maggioni, and B. Nadler: Diffusion maps, reduction coordinates and low dimensional representation of stochastic systems. - Multiscale Model. Simul. 7:2, 2008, 842-864.

[10] Colfman, R. R., and S. Lafon: Diffusion maps. - Appl. Comput. Harmon. Anal. 21:1, 2006, $5-30$. 
[11] Coifman, R.R., and S. LAfon: Geometric harmonics: a novel tool for multiscale out-ofsample extension of empirical functions. - Appl. Comput. Harmon. Anal. 21:1, 2006, 31-52.

[12] Coifman, R. R., S. Lafon, A. B. Lee, M. Maggioni, B. Nadler, F. Warner, and S. W. ZUCKER: Geometric diffusions as a tool for harmonic analysis and structure definition of data: Diffusion maps. - Proc. Natl. Acad. Sci. USA 102:21, 2005, 7426-7431.

[13] Coifman, R. R., S. Lafon, A. B. Lee, M. Maggioni, B. Nadler, F. Warner, and S. W. ZUCKER: Geometric diffusions as a tool for harmonic analysis and structure definition of data: Multiscale methods. - Proc. Natl. Acad. Sci. USA 102:21, 2005, 7432-7437.

[14] Coifman, R. R., and M. Maggioni: Diffusion wavelets. - Appl. Comput. Harmon. Anal. $21: 1,2006,53-94$.

[15] Davies, E. B.: Spectral properties of compact manifolds and changes of metric. - Amer. J. Math. 112:1, 1990, 15-39.

[16] Davies, E. B.: Heat kernels and spectral theory. - Cambridge Univ. Press, 1989.

[17] Donnelly, H., and C. Fefferman: Growth and geometry of eigenfunctions of the Laplacian. - In: Analysis and Partial Differential Equations, Lecture Notes in Pure and Appl. Math. 122, Dekker, New York, 1990, 635-655.

[18] Donoho, D. L., and C. GRImes: When does isomap recover natural parameterization of families of articulated images? - Technical Report 2002-27, Department of Statistics, Stanford University, 2002.

[19] Donoho, D. L., and C. Grimes: Hessian eigenmaps: new locally linear embedding techniques for high-dimensional data. - Proc. Natl. Acad. Sci. USA 100:10, 2003, 5591-5596.

[20] Donoho, D. L., O. Levi, J.-L. Starck, and V. J. Martinez: Multiscale geometric analysis for 3-d catalogues. - Technical Report, Stanford Univ., 2002.

[21] Dоов, J. L.: Classical potential theory and its probabilistic counterpart. - Springer-Verlag, New York, 1984.

[22] Federer, H.: Geometric measure theory. - Springer-Verlag, 1969.

[23] Gallot, S., D. Hulin, and J. Lafontaine: Riemannian geometry. - Springer-Verlag, Berlin, 1987.

[24] GrÜter, M., and K.-O. Widman: The Green function for uniformly elliptic equations. Manuscripta Math. 37, 1982, 303-342.

[25] He, X., S. Yan, Y. Hu, P. NiYogi, and H.-J. Zhang: Face recognition using Laplacian faces. - IEEE Trans. Pattern Analysis and Machine Intelligence 27:3, 2005, 328-340.

[26] Hempel, R., L. Seco, and B. Simon: The essential spectrum of Neumann Laplacians on some bounded singular domains. - J. Funct. Anal. 102:2, 1991, 448-483.

[27] Jones, P. W., M. Maggioni, and R. Schul: Manifold parametrizations by eigenfunctions of the Laplacian and heat kernels. - Proc. Natl. Acad. Sci. USA 105:6, 2008, 1803-1808.

[28] KellogG, O. D.: Foundations of potential theory. - Berlin, 1929.

[29] Lafon, S.: Diffusion maps and geometric harmonics. - PhD thesis, Yale University, Dept. of Mathematics \& Applied Mathematics, 2004.

[30] Lo, P.-C.: Three dimensional filtering approach to brain potential mapping. - IEEE Trans. on Biomedical Engineering 46:5, 1999, 574-583.

[31] Mahadevan, S., K. Ferguson, S. Osentoski, and M. Maggioni: Simultaneous learning of representation and control in continuous domains. - In: Association for the Advancement of Artificial Intelligence (AAAI), AAAI Press, 2006.

[32] Mahadevan, S., and M. Maggioni: Value function approximation with diffusion wavelets and Laplacian eigenfunctions. - Advances in Neural Information Processing Systems 18, 2005. 
[33] Netrusov, Yu.: Sharp remainder estimates in the weyl formula for the Neumann Laplacian on a class of planar regions. - J. Funct. Anal. 250:1, 2007, 21-41.

[34] Netrusov, Yu., and Yu. Safarov: Weyl asymptotic formula for the Laplacian on domains with rough boundaries. - Comm. Math. Phys. 253:2, 2005, 481-509.

[35] NG, A., M. JoRdAn, and Y. Weiss: - On spectral clustering: Analysis and an algorithm. Advances in Neural Information Processing Systems 14, 2002.

[36] Niyogi, P., I. Matveeva, and M. Belkin: Regression and regularization on large graphs. Technical Report, University of Chicago, 2003.

[37] Øksendal, B.: Stochastic differential equations. - Universitext, Springer-Verlag, 1985.

[38] Pommerenke, Ch.: Univalent functions. - Vandenhoeck \& Ruprecht, Göttingen, 1975. With a chapter on quadratic differentials by G. Jensen, Mathematische Lehrbücher, Band XXV.

[39] Roweis, S. T., and L. K. SAUL: Nonlinear dimensionality reduction by locally linear embedding. - Science 290, 2000, 2323-2326.

[40] Saul, L. K., K. Q. Weinberger, F. H. Ham, F. Sha, and D. D. Lee: Spectral methods for dimensionality reduction (chapter Semisupervised learning). - MIT Press, 2006.

[41] SHA, F., and L. K. SAUL: Analysis and extension of spectral methods for nonlinear dimensionality reduction. - In: Proc. International Conference on Machine Learning, 2005, 785-792.

[42] Shen, X., and F. G. Meyer: Analysis of event-related fmri data using diffusion maps. - In: Proc. Information Processing in Medical Imaging, 2005, 652-663.

[43] SHI, J., and J. MALIK: Normalized cuts and image segmentation. - IEEE Pattern Analysis and Machine Intelligence 22, 2000, 888-905.

[44] Sмith, H. F.: Sharp $l^{2}-l^{q}$ bounds on spectral projectors for low regularity metrics. - Math. Res. Lett. 13:6, 2006, 967-974.

[45] Smith, H.F.: Spectral cluster estimates for $c^{1,1}$ metrics. - Amer. J. Math. 128, 2006, 10691103.

[46] Sogge, C. D.: Eigenfunction and Bochner-Riesz estimates on manifolds with boundary. Math. Res. Lett. 9, 2002, 205-216.

[47] Spielman, D. A., and S.H. Teng: Spectral partitioning works: Planar graphs and finite element meshes. - Foundations of Computer Science, 1996.

[48] Stroock, D. W.: Partial differential equations for probabilists. - Cambridge Stud. Adv. Math. 112, Cambridge Univ. Press, Cambridge, 2008.

[49] Stroock. D. W.: Diffusion semigroups corresponding to uniformly elliptic divergence form operators. - Séminaire de Probabilités 22, 1988, 316-347.

[50] Szlam, A. D., M. Maggioni, and R. R. Coifman: A general framework for adaptive regularization based on diffusion processes on graphs. - Technical Report YALE/DCS/TR1365, Yale Univ., 2006 (submitted).

[51] Szlam, A. D., M. Maggioni, R. R. Coifman, and J. C. Bremer JR.: Diffusion-driven multiscale analysis on manifolds and graphs: top-down and bottom-up constructions. - In: Proc. SPIE 5914-1, 2005.

[52] Tenenbaum, J. B., V. De Silva, and J. C. Langford: A global geometric framework for nonlinear dimensionality reduction. - Science 290, 2000, 2319-2323.

[53] Weinberger, K. Q., and L. K. Saul: An introduction to nonlinear dimensionality reduction by maximum variance unfolding. - In: Proc. Association for the Advancement of Artificial Intelligence (AAAI), 2006.

[54] Weinberger, K. Q., F. Sha, and L. K. SAul: Leaning a kernel matrix for nonlinear dimensionality reduction. - In: Proc. International Conference on Machine Learning, 2004, 839-846. 
[55] Wiener, M.: - J. Math. Phys. M.I.T. 3, 1924, 24-51, 127-146.

[56] XU, X.: New proof of the Hörmander multiplier theorem on compact manifolds without boundary. - Proc. Amer. Math. Soc. 135:5, 2007, 1585-1595.

[57] Zhang, Z. and H. ZHA: Principal manifolds and nonlinear dimension reduction via local tangent space alignement. - Technical Report CSE-02-019, Dept. of Computer Science and Engineering, Pennsylvania State Univ., 2002.

Received 27 October 2008 